\title{
HYDRODYNAMIC ANALYSIS OF A LIQUID-SOLID FLUIDIZED BED VIA CFD-DEM SIMULATIONS, STABILITY ANALYSIS, AND TOMOGRAPHY
}

By

\author{
Hussein Zbib \\ B.Eng. Ryerson University, Toronto, Canada, 2016
}

\author{
A thesis \\ presented to Ryerson University \\ in partial fulfilment of the requirements for the degree of \\ Master of Applied Science \\ in the program of Chemical Engineering
}

Toronto, Ontario, Canada, 2018

(C) Hussein Zbib, 2018 


\section{AUTHOR'S DECLERATION FOR ELECTRONIC SUBMISSION OF A THESIS}

I hereby declare that I am the sole author of this thesis. This is a true copy of the thesis, including any required final revisions, as accepted by my examiners.

I authorize Ryerson University to lend this thesis to other institutions or individuals for the purpose of scholarly research.

I further authorize Ryerson University to reproduce this thesis by photocopying or by other means, in total or in part, at the request of other institutions or individuals for the purpose of scholarly research.

I understand that my thesis may be made electronically available to the public. 


\begin{abstract}
Hussein Zbib

Hydrodynamic Analysis of a Liquid-Solid Fluidized Bed via CFD-DEM Simulations, Stability Analysis, and Tomography
\end{abstract}

\title{
MASc, Chemical Engineering, Ryerson University, Toronto, 2018
}

A coupled computational fluid dynamics (CFD) and discrete element method (DEM) model was developed to analyze the fluid-particle and particle-particle interactions in a 3D liquid-solid fluidized bed (LSFB). The CFD-DEM model was validated using the Electrical Resistance Tomography (ERT) experimental method. ERT was employed to measure the bed-averaged particle volume fraction (BPVF) of $0.002 \mathrm{~m}$ glass beads fluidized with water for various particle numbers and flow rates. It was found that simulations employing the combination of the Gidaspow drag model with pressure gradient and virtual mass forces provided the least percentage error between experiments and simulations. It was also found that contact parameters must be calibrated to account for the particles being wet. The difference between simulations and experiments was $4.74 \%$. The CFD-DEM model was also employed alongside stability analysis to investigate the hydrodynamic behavior within the LSFB and the intermediate flow regime for all cases studied. 


\section{Acknowledgements}

I am truly blessed, and this is all the doing of a merciful creator. Thank you for guiding my heart to act decently, and thank you for allowing my mind to simply think.

I would like to express my utmost gratitude and appreciation to my supervisors Dr. Farhad Ein-Mozaffari and Dr. Ali Lohi. Thank you for your guidance, encouragement, support, and motivation.

My deepest gratitude goes to Dr. Mohammadreza Ebrahimi for his mentorship and patience. Your contribution to my learning and academic development is forever appreciated.

Endless thanks to Dr. Ginette Turcotte. Thank you for helping me persevere through very difficult times during my undergraduate degree and leading up to masters.

Special thanks to Mr. Ali Hemmati, Mr. Daniel Boothe, and Mr. Tondar Tajrobekar for their help in designing and building of the experimental setup. Also, thanks go to the Chemical Engineering staff: Ms. Louise Lichacz, Ms. Goretti Praticante, and Ms. Jessica Miniaci for all their help.

Lastly, I would like to express my gratitude to my fellow graduates and friends in the Chemical Engineering department. You have helped me in more ways than you can imagine. 
To the most Beautiful

My mother, Youssra

My siblings, Ghassan, Maya, Katia, and Ali

For your endless love 


\section{Table of Contents}

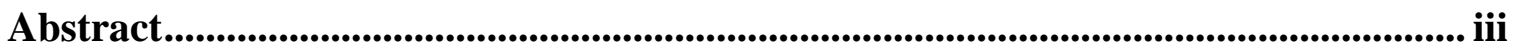

Acknowledgements .............................................................................................................................. iv

Table of Contents ............................................................................................................................... vi

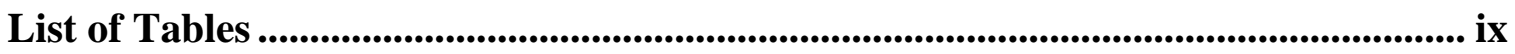

List of Figures.................................................................................................................................. ix

Nomenclature .......................................................................................................................................... xi

Introduction.....................................................................................................................................1

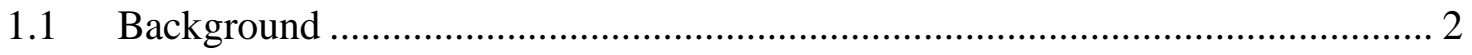

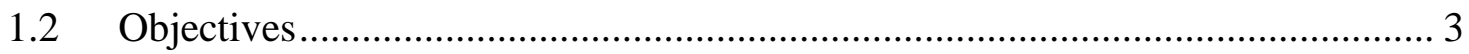

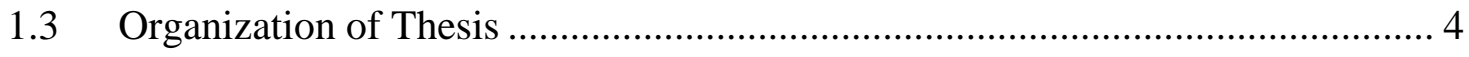

Literature Review ......................................................................................................................................5

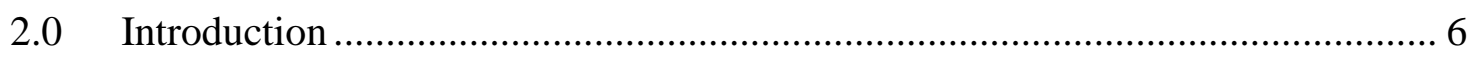

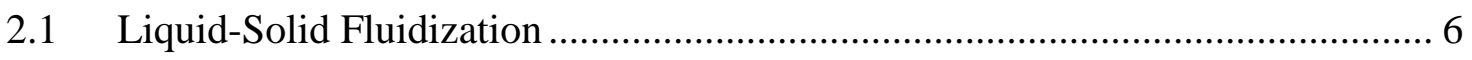

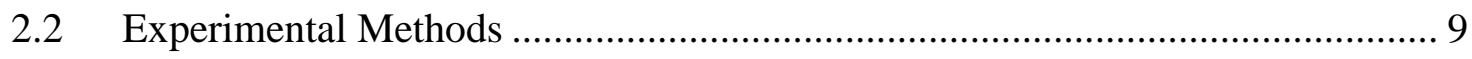

2.2.1 Particle Image Velocimetry …………………………………………... 10

2.2.2 Radioactive Particle Tracking .................................................................. 10

2.2.3 Positron Emission Particle Tracking ............................................................ 11

2.2.4 Laser Doppler Anemometry / Phase Doppler Anemometry ......................... 11

2.2.5 Electrical Resistance Tomography ……………….................................. 12

2.2.6 Electrical Capacitance Tomography ………………………..................... 16

2.2.7 Concluding Remarks.............................................................................. 17

2.3 The Numerical Approach …………………….............................................. 17

2.3.2 Discrete Element Method: Governing Equations …………......................... 18

2.3.3 Computational Fluid Dynamics: Governing Equations …………………... 21 


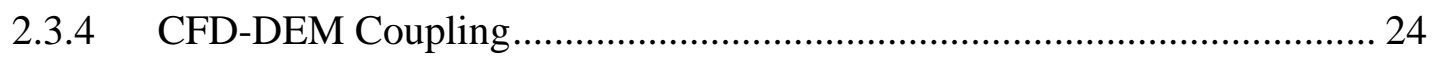

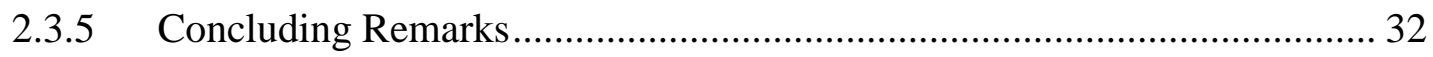

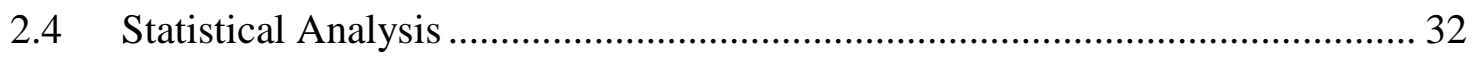

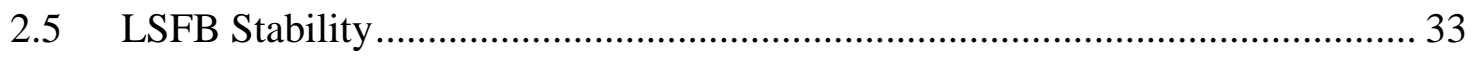

2.6 Application of CFD-DEM in LSFB Literature ......................................... 35

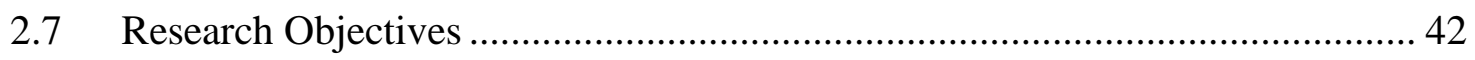

Experimental and Simulation Methodologies ..........................................................43

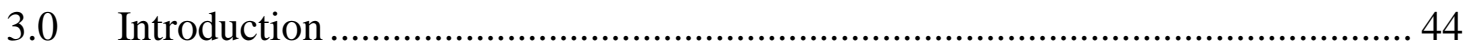

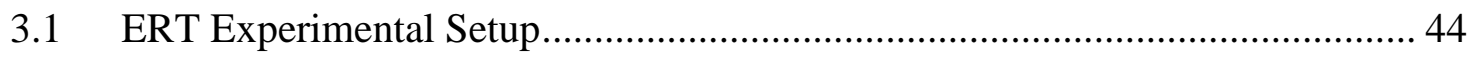

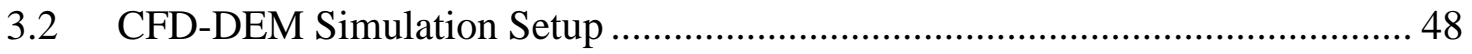

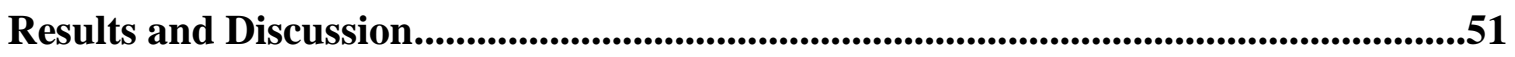

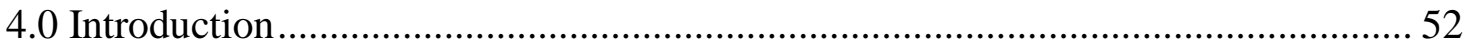

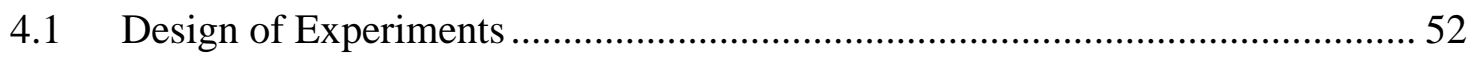

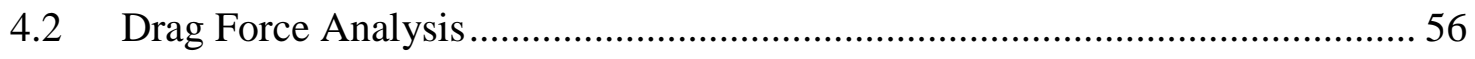

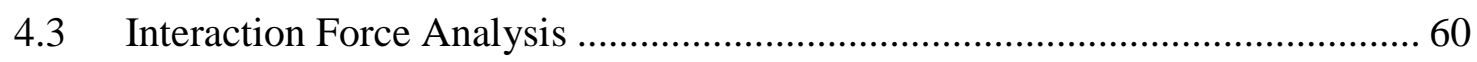

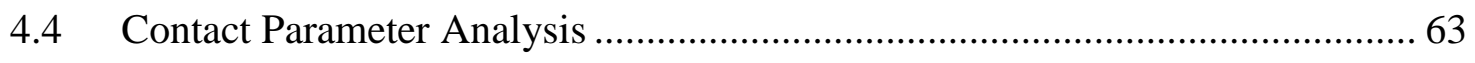

4.5 Local Comparison between Simulation and Experimental Results .................. 68

4.6 The Influence of Liquid Superficial Velocity on System Hydrodynamics ........ 69

4.6.1 Stability Analysis for Various Liquid Superficial Velocities .................... 69

4.6.2 The Effect of Liquid Superficial Velocity on the Liquid and Particle Flow

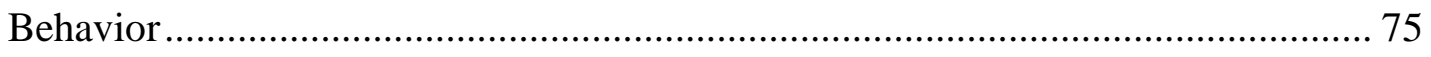

4.7 The Effect of Number of Particles on System Hydrodynamics ....................... 79

4.7.1 Stability Analysis for Various Number of Particles ............................... 79

4.7.2 The Effect of the Number of Particles on the Liquid and Particle Flow

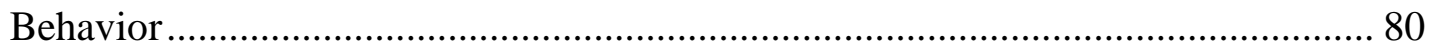

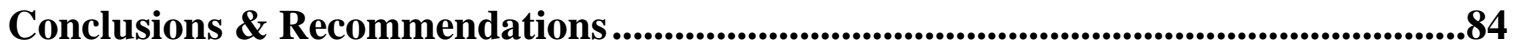




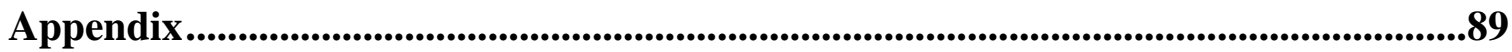

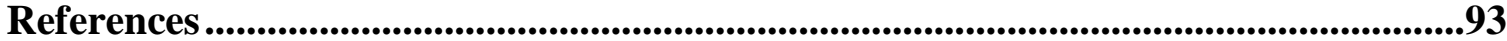




\section{List of Tables}

Table 1: Interaction forces as included in literature of CFD-DEM simulations of LSFB ….......... 37

Table 2: CFD-DEM studies on liquid-solid fluidized beds published to date............................... 41

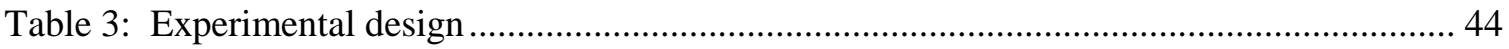

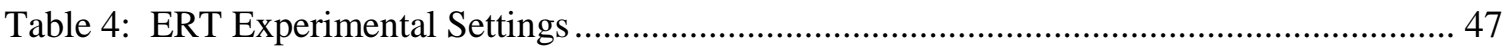

Table 5: Particle, geometry, and Fluid properties .................................................................... 49

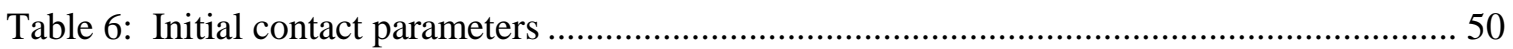

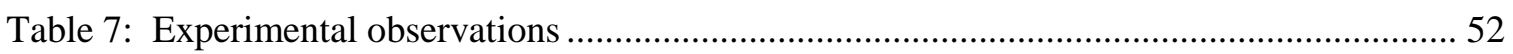

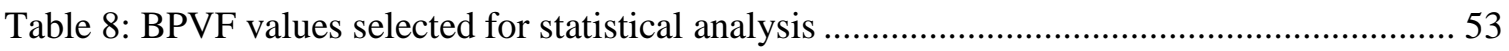

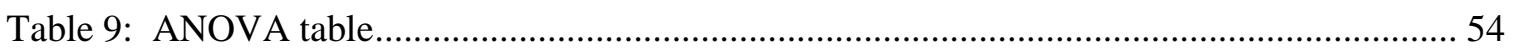

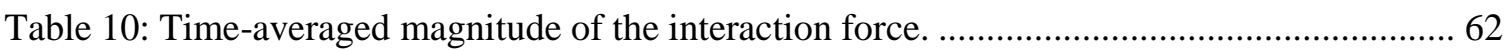

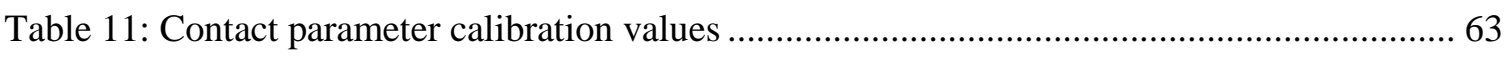

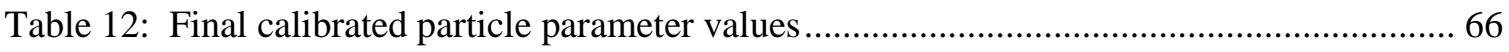

\section{List of Figures}

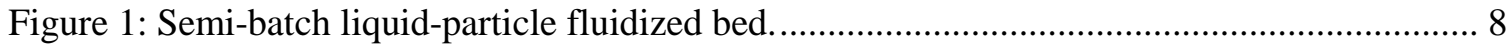

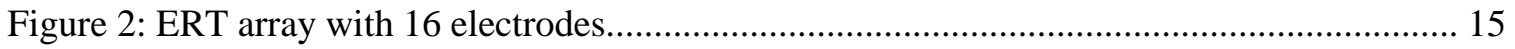

Figure 3: One-way, two-way, and four-way coupling (Van der Hoef et al., 2006)...................... 30

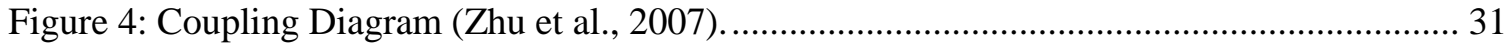

Figure 5: The various achievable flow regimes for particles of different densities and diameters

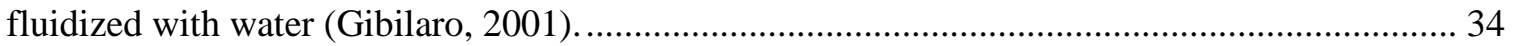

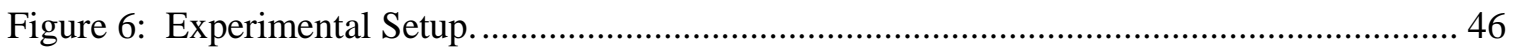

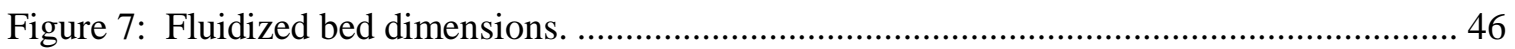

Figure 8: Instantaneous ERT results for 50,000, $0.002 \mathrm{~m}$ particles, fluidized at $0.20 \mathrm{~m} / \mathrm{s}$ at $2.5 \mathrm{~s}$, $5.0 \mathrm{~s}, 7.5 \mathrm{~s}$, and $10.0 \mathrm{~s}$ timed pursuant to visual confirmation of steady state conditions. ............ 47

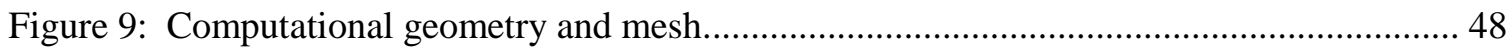

Figure 10: Model prediction as compared to actual results for $0.002 \mathrm{~m}$ particle fluidization....... 54

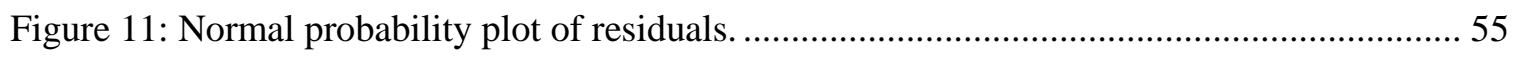

Figure 12: 3D Surface of the $0.002 \mathrm{~m}$ particle fluidization predicted model................................ 56

Figure 13: Experimental image of particle position during the fluidization of 50,000 particles

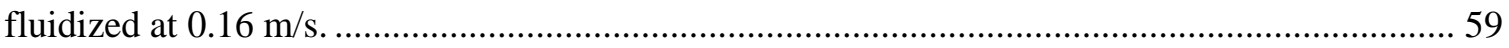

Figure 14: Comparison between fluidized bed height predicted by various drag models for $50,000,0.002 \mathrm{~m}$ particles, at $0.16 \mathrm{~m} / \mathrm{s}$ a) Schiller-Naumann b) Gidaspow c) Symlal-O'brien..... 59 
Figure 15: Quantitative comparison of PPVF (\%) between experimental runs and simulations using various drag models.

Figure 16: The influence of the inclusion of various interaction forces on simulation results (a) Gidaspow Drag Only (b) Drag + Pressure gradient (Pg) (c) Drag + Pg + Virtual mass (Vm) (d)

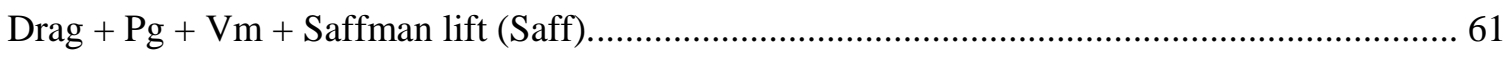

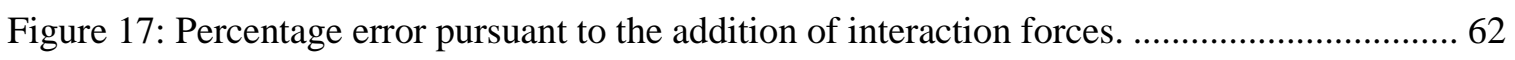

Figure 18: Coefficient of restitution calibration a) COR P-W b) COR P-P ................................. 64

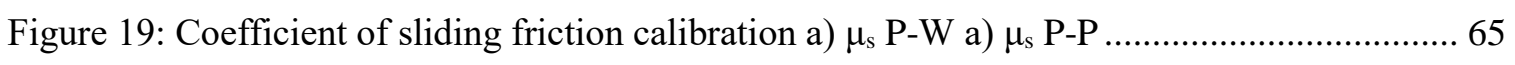

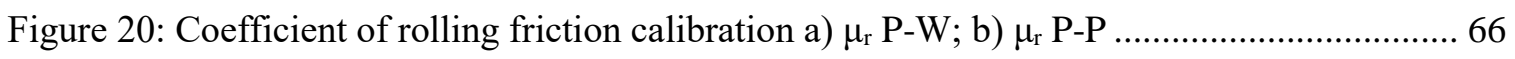

Figure 21: Summary of interaction force analysis and contact parameters calibration................ 67

Figure 22: Comparison between experimental and simulation PVF in the radial direction $\left(0^{\circ}\right.$ angle) at $0.12 \mathrm{~m}$ height, for 50,000 particles at $0.16 \mathrm{~m} / \mathrm{s}$. (Percentages above the data points represent the percentage error between simulations and experiments at the prevailing dimensionless radius).

Figure 23: Instantaneous snapshots of 50,000 particles fluidized at a) $0.12 \mathrm{~m} / \mathrm{s} \mathrm{b}) 0.16 \mathrm{~m} / \mathrm{s}$ and c) $0.20 \mathrm{~m} / \mathrm{s}$. 70

Figure 24: Stability as a function of porosity for $0.002 \mathrm{~m}$ glass beads fluidized with water.

Porosities calculated from the CFD-DEM results at various flowrates are indicated by colored

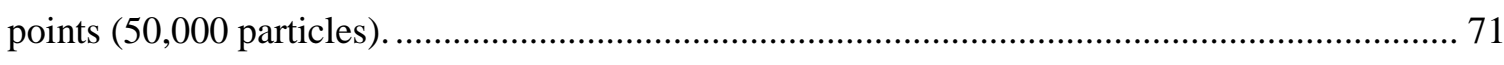

Figure 25: PVF variation along the bed height at various liquid superficial velocities................. 72

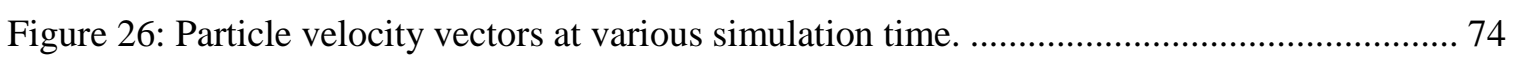

Figure 27: Time-averaged liquid velocity contour along the bed height with 50,000 particles. a) $0.16 \mathrm{~m} / \mathrm{s} \mathrm{c}) 0.20 \mathrm{~m} / \mathrm{s}$. 75

Figure 28: (a) Liquid MAV, (b) particle MAV and (c) PVF along the radial direction for 50,000 particles fluidized at various liquid superficial velocities calculated at $0.12 \mathrm{~m}$ bed height. 78

Figure 29: Stability as a function of porosity for $0.002 \mathrm{~m}$ glass beads fluidized with water.

Porosities calculated from the CFD-DEM results at various number of particles are indicated by colored points.

Figure 30: (a) Liquid MAV, (b) particle MAV and (c) PVF along the radial direction for various number of particles fluidized at $0.16 \mathrm{~m} / \mathrm{s}$ liquid superficial velocity calculated at $0.12 \mathrm{~m}$.......... 83

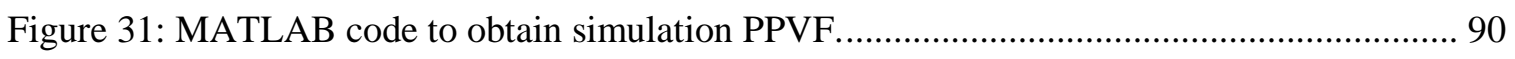

Figure 32: Gulf Streaming and Vortices for 50,000 and 75,000 particles fluidized at $0.16 \mathrm{~m} / \mathrm{s} . .91$

Figure 33: Gulf Streaming and Vortex dominated flows for 100,000 particles fluidized at 0.16 $\mathrm{m} / \mathrm{s}$. 


\section{Nomenclature}

\section{Latin Symbols}

\begin{tabular}{|c|c|}
\hline$A_{c}$ & Cross sectional area $\left[\mathrm{m}^{2}\right]$ \\
\hline$A r$ & Archimedes number \\
\hline$A$ & Hamaker constant \\
\hline c & Damping constant \\
\hline$C_{d}$ & Drag coefficient \\
\hline$C_{n}$ & Normal damping coefficient \\
\hline $\mathrm{C}_{\mathrm{r}}$ & Viscous damping constant \\
\hline$C_{t}$ & Tangential damping coefficient \\
\hline$C_{V m}$ & Virtual mass coefficient \\
\hline$d_{p}$ & Particle diameter $[\mathrm{m}]$ \\
\hline$\frac{-d p_{f}}{d z}$ & Frictional pressure gradient $\left[\mathrm{N} / \mathrm{m}^{3}\right]$ \\
\hline$e_{r}$ & Restitution coefficient \\
\hline$E^{*}$ & Reduced Young's modulus [N/m³] \\
\hline $\boldsymbol{E}_{i j}$ & Mean rate of deformation $\left[\mathrm{s}^{-1}\right]$ \\
\hline $\boldsymbol{f}_{e}$ & Spring force $[\mathrm{N}]$ \\
\hline $\boldsymbol{f}_{d}$ & Damping force $[\mathrm{N}]$ \\
\hline$f_{n, e}$ & $\begin{array}{l}\text { Elastic component of normal interaction between } \\
\text { particles }\end{array}$ \\
\hline $\boldsymbol{F}_{\text {Basset }}$ & Basset force $[\mathrm{N}]$ \\
\hline $\boldsymbol{F}_{i j^{c}}$ & Contact force acting on particle $\mathrm{i}$ by particle $\mathrm{j}[\mathrm{N}]$ \\
\hline $\boldsymbol{F}_{d}$ & Drag force $[\mathrm{N}]$ \\
\hline $\boldsymbol{F}^{e}$ & Electrostatic force $[\mathrm{N}]$ \\
\hline $\boldsymbol{F}_{i}^{g}$ & Gravitational force on particle i $[\mathrm{N}]$ \\
\hline $\boldsymbol{f}_{i}^{f}$ & Particle-fluid interaction force $[\mathrm{N}]$ \\
\hline$F_{i}^{f}$ & Fluid-particle interaction force $[\mathrm{N}]$ \\
\hline $\boldsymbol{F}_{i, \text { avg }}^{f}$ & $\begin{array}{l}\text { Fluid-particle interaction force in a computational } \\
\text { cell }[\mathrm{N}]\end{array}$ \\
\hline $\boldsymbol{F}_{\text {Mag }}$ & Magnus lift force $[\mathrm{N}]$ \\
\hline $\boldsymbol{F}^{l}$ & Capillary force $[\mathrm{N}]$ \\
\hline $\boldsymbol{F}_{i j}^{n}$ & Normal force $[\mathrm{N}]$ \\
\hline $\boldsymbol{F}_{p}$ & Pressure gradient force $[\mathrm{N}]$ \\
\hline $\boldsymbol{F}_{\text {Saff }}$ & Saffman lift force $[\mathrm{N}]$ \\
\hline $\boldsymbol{F}_{i j}^{t}$ & Tangential force $[\mathrm{N}]$ \\
\hline $\boldsymbol{F}_{V m}$ & Virtual mass force $[\mathrm{N}]$ \\
\hline $\boldsymbol{F}^{v}$ & Van der Waals force $[\mathrm{N}]$ \\
\hline $\mathrm{G}^{*}$ & Equivalent shear modulus $\left[\mathrm{N} / \mathrm{m}^{2}\right]$ \\
\hline$h$ & Surface gap between two spheres $[\mathrm{m}]$ \\
\hline$g$ & Acceleration due to gravity $\left[\mathrm{m} / \mathrm{s}^{2}\right]$ \\
\hline $\boldsymbol{I}_{\mathrm{i}}$ & Moment of inertia $\left[\mathrm{kg} / \mathrm{m}^{2}\right]$ \\
\hline$k$ & Turbulent kinetic energy $[\mathrm{J}]$ \\
\hline$k_{c}$ & Number of particles in a computational cell \\
\hline$k_{p}$ & Particle stiffness \\
\hline$k_{r}$ & Rolling stiffness \\
\hline $\mathrm{L}$ & Bed height $[\mathrm{m}]$ \\
\hline$m_{i}$ & Mass of particle i [kg] \\
\hline $\mathrm{m}$ & Mass of all particles $[\mathrm{kg}]$ \\
\hline$m^{*}$ & Reduced mass of the particle [kg] \\
\hline M & Total number of measurements (ERT) \\
\hline
\end{tabular}

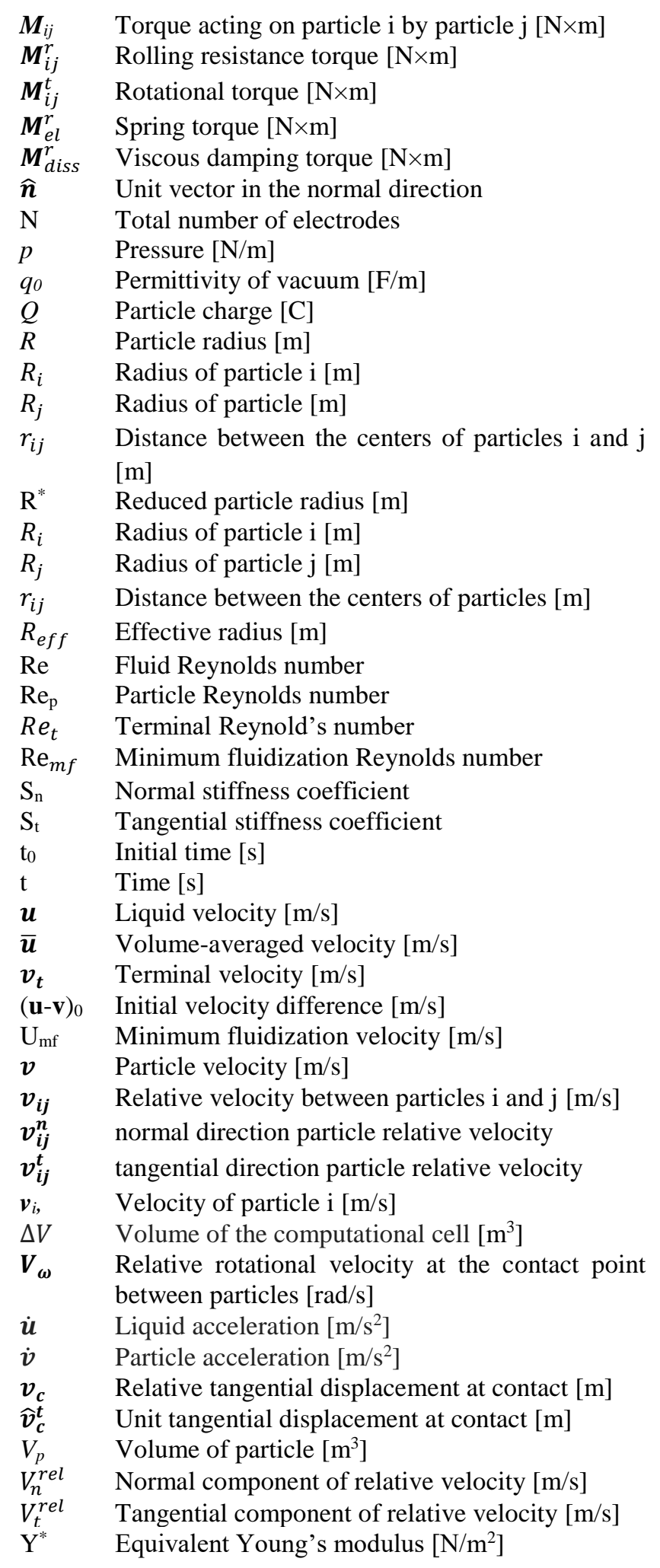


Greek Symbols

\begin{tabular}{|c|c|}
\hline$\zeta$ & Energy dissipation constant \\
\hline$\delta$ & Overlap $[\mathrm{m}]$ \\
\hline$\delta_{n}$ & Normal overlap $[\mathrm{m}]$ \\
\hline$\delta_{t}$ & Tangential overlap $[\mathrm{m}]$ \\
\hline$\delta_{\max }$ & $\begin{array}{l}\text { Maximum tangential displacement when the } \\
\text { particles start to slide. [m] }\end{array}$ \\
\hline$\beta_{p f}$ & Momentum exchange coefficient \\
\hline$\varepsilon$ & Dissipation of turbulent kinetic energy [J] \\
\hline$\varepsilon_{f}$ & Fluid volume fraction \\
\hline$\varepsilon_{s}$ & Particle volume fraction \\
\hline$\varepsilon_{m f}$ & Minimum fluidization porosity \\
\hline$\eta_{r}$ & Viscous damping ratio of rolling torque \\
\hline$\mu_{f}$ & Fluid viscosity [Pa×s] \\
\hline$\mu_{r}$ & Rolling friction coefficient \\
\hline$\mu_{s}$ & Sliding friction coefficient \\
\hline$\mu_{t}$ & turbulent viscosity $[\mathrm{Pa} \times \mathrm{s}]$ \\
\hline$v$ & Poisson's ratio \\
\hline$\rho_{f}$ & Fluid density $\left[\mathrm{kg} / \mathrm{m}^{3}\right]$ \\
\hline$\tau$ & Volume-average stress tensor $[\mathrm{Pa}]$ \\
\hline$\theta$ & Contact angle between two particles [rad] \\
\hline$\Delta \theta_{r}$ & $\begin{array}{l}\text { Incremental relative rotation between two } \\
\text { particles [rad] }\end{array}$ \\
\hline$\frac{d \theta_{r}}{d t}$ & $\begin{array}{l}\text { Rate of change in the relative rotation between } \\
\text { two particles in contact }[\mathrm{rad} / \mathrm{s}]\end{array}$ \\
\hline$\varphi_{p}$ & Sphericity \\
\hline$\omega_{c}$ & vorticity $\left[\mathrm{s}^{-1}\right]$ \\
\hline$\omega_{d}$ & Particle rotation $\left[\mathrm{s}^{-1}\right]$ \\
\hline$\omega_{i}$ & Angular velocity of particle I [rad/s] \\
\hline$\psi_{i j}$ & Unit vector of relative angular velocity \\
\hline$\psi_{i}$ & Unit vector of angular velocity for particle i \\
\hline$\psi_{j}$ & Unit vector of angular velocity for particle $j$ \\
\hline$\gamma$ & Liquid surface tension $[\mathrm{N} / \mathrm{m}]$ \\
\hline
\end{tabular}

\section{Subscripts}

$i \quad$ particle $\mathrm{i}$

$j \quad$ particle $\mathrm{j}$

$p \quad$ particle

$f \quad$ fluid 


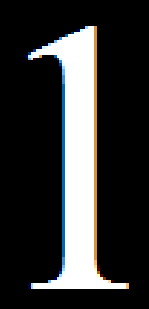

Introduction 


\subsection{Background}

Liquid solid fluidization is a process characterized by the suspension of particles in an upwards flowing liquid. Once suspended, the particles are said to be "fluidized". Liquid-Solid fluidized beds (LSFBs) are highly prevalent in the chemical, petrochemical, and pharmaceutical industries (Esteghamatian et al., 2017).

LSFBs are attractive to processes that require operation at high fluid superficial velocities while maintaining a relatively homogeneous (spatially equal) distribution of particles. The uniformity results in high and equal rates of heat and mass transfer between the two phases throughout the system. This flow regime is commonly referred to as the homogeneous regime. This characteristic is specific to LSFBs as high fluid superficial velocities may cause other systems (i.e. gas-solid fluidized beds) to "bubble". Bubbling occurs when large pockets (or bubbles) of a fluid propagate from the bottom of the bed towards the top, creating high porosity regions as they axially displace. This phenomenon causes greater, however, uneven heat and mass transfer rates throughout the system. The bubbling flow regime is achievable in certain LSFB systems, however, industrial LSFB processes rarely operate in this condition (Epstein, 2003). Alternatively, it is possible to operate LSFBs within an intermediate stability region, characterized by non-bubbling and nonhomogeneous flows. Flows in this regime can provide a compromise between the homogeneity and rates of heat and mass transfer. Thus, understanding the flow behavior in the intermediate stability flow regime is greatly beneficial to processes that require high exchange rates in transport phenomena, without drastically diminishing the evenness of the exchange.

Generally, LSFBs are designed through analytical and experimental methods. Experiments, however, carry their respective cost such as capital, material, manpower, and time. Computer simulations have increasingly become popular in recent years as an alternative or complementary tool to pilot scale experiments. They are particularly very valuable means when experiments are expensive or when it is desired to quantify particle-scale phenomena that may be impossible to determine experimentally. The accuracy (or viability) of computer simulations ultimately depends on how closely these simulations resemble real life phenomena. Therefore, computer simulations must be initially validated with experimental data. The validated model can then be used for optimization and scale up purposes (Tu et al., 2013). 
One of the most common experimental measurement techniques employed for liquid-solid (twophase) systems is the Electrical Resistance Tomography (ERT). Its popularity is due to its nonintrusive nature, and its ability to provide visualizations of particle concentrations (Kazemzadeh, 2016; Pakzad et al., 2008; Hosseini et al., 2010; Mishra \& Ein-Mozaffari, 2016; Mishra \& EinMozaffari, 2017). Furthermore, to analyze processes experimentally, statistical design of experiments is often employed. By utilizing statistical methods, the effect of process variables and their interaction on the experimental output can be quantified; this allows for comprehensive process analysis and optimization (Pakzad et al. 2013; Ghafoori et al., 2014; Kirmizakis et al., 2014; Pakzad \& Azimi, 2017; Kazemzadeh et al., 2017a).

Many numerical methods have been used to simulate the flow within LSFBs. However, one very popular numerical method is the coupled Computational Fluid Dynamics-Discrete Element Method (CFD-DEM) approach. The CFD-DEM approach is often selected due to the compromise it provides between the computational time and the level of detail. In the CFD-DEM method, the Eulerian approach is applied to model the liquid by solving the locally averaged Navier-Stokes equations, and the Lagrangian approach is applied to model the solid by solving Newton's second law. The two phases are coupled through Newton's third law as the interaction forces between the two phases are equal in magnitude and opposite in direction. By studying a LSFB through CFDDEM simulations, it is possible to quantify the effect of particle-scale phenomena (such as liquidparticle interaction forces and particle-particle contact force) on the overall macroscopic behavior of the bed. This provides invaluable information which can be employed for LSFB process optimization and design.

\subsection{Objectives}

The principal objective of this study is to comprehensively investigate a 3D LSFB via tomography experiments, statistical modeling, CFD-DEM simulations, and stability analysis. ERT experiments are employed to validate the developed CFD-DEM simulations. The CFD-DEM model is employed to quantify the influence of interaction forces, and particle contact parameters on the simulation results. Furthermore, stability analysis is employed alongside the CFD-DEM simulation results to identify the flow regimes for the LSFB system under investigation at various number of particles and fluid superficial velocities. This analysis provides valuable insight into the effect of the aforementioned variables on the hydrodynamic behavior within the LSFB. 


\subsection{Organization of Thesis}

The current study is organized as follows: Chapter two briefly covers the concept of Liquid-solid fluidization, the experimental measurement techniques applied for two-phase systems, the mathematical equations applied in the CFD-DEM coupling method, and statistical and stability analyses. Chapter two also summarizes the literature review of the application of the CFD-DEM approach in the simulation of LSFBs. Chapter three presents the research methodology including experimental and CFD-DEM simulation setups. Chapter four presents the ERT experimental results and statistical analysis. The CFD-DEM simulation validation is also covered in this chapter with a detailed investigation of the influence of particle-particle and particle-fluid interaction on the simulation results. Furthermore, chapter four presents the LSFB stability analysis and discusses the effect of varying liquid superficial velocity and number of particles on the hydrodynamic behavior of the system. Finally, chapter five concludes the research results and provides recommendations for future work. 
Literature

Review 


\subsection{Introduction}

This chapter presents fundamental concepts, established theory, and the application of CFD-DEM simulations of LSFBs currently available in literature. Section 2.1 introduces liquid-solid fluidization and presents fundamental concepts upon which the design of LSFBs is commonly built. Section 2.2 presents the various experimental methods which are often employed to study LSFBs. This section also explains in considerable detail the fundamentals of electrical resistance tomography (selected for this study). Section 2.3 discusses the numerical approach employed in this research (the coupled CFD-DEM approach); this section introduces CFD, DEM, and CFDDEM coupling. Section 2.4 briefly discusses statistical methods often employed to study systems with multiphase flow. Section 2.5 presents the LSFB stability concept; this concept can be used to identify the flow regime within a LSFB. Section 2.6 presents all available literature which applied CFD-DEM simulations to study LSFBs; this section highlights the gaps in literature, upon which the novelty of this work is based. Finally, section 2.7 presents the research objectives.

\subsection{Liquid-Solid Fluidization}

Fluidization is best explained through a practical example. Consider a cylindrical column filled with water, with a single spherical solid particle resting at the bottom. The forces acting on the particle are: an upwards buoyant force and a downwards gravitational force. A very logical conclusion can now be made: The fact that the solid is resting at the bottom of the cylinder indicates that the gravitational force is greater than the buoyant force. Now, consider the same column with perforated top and bottom portions, connected to an outside pump and piping system which allows circulation of water into and out of the column. When the pump is turned on, and water is circulated from the bottom to the top of the column, the fluid-particle interaction force is introduced to the particle. This force is directly proportional to the fluid flow rate. If the flow rate is increased to a certain minimum, the fluid-particle interaction force will overcome the weight of the particle and 'lift' the solid from the bottom of the column. The particle is thus said to be 'fluidized' at the 'minimum fluidization velocity' and, hence, the definition of fluidization is: The suspension of a particle(s) within an upwards flowing fluid (Kunii \& Levenspiel, 1991).

The above definition can be easily extended to a bed of solid particles using the same concept of balancing gravitational and the fluid-particle interaction force. The application of fluidized beds is abundant in industry due to a multitude of benefits, however the vast majority of research studies 
and industrial applications in the fluidization field involve gas-solid systems. As an example, in 1992, at the international conference of fluidization, only two out of one hundred papers presented research regarding liquid-solid fluidized beds (Potter \& Nicklin, 1992). This is because there are far more tangible industrial applications to gas beds than their counterpart (Di Felice, 1995). The case for liquid beds lies in recent development of LSFB applications such as: electrolysis, bioreactions, and heat exchange (Epstein, 2002), particularly because these beds exhibit a "homogeneous, smooth expansion" of particles (vs. the bubbling behavior seen in gas beds) (Kunii \& Levenspiel, 1991).

There are two types of LSFBs: circulating and semi-batch. In circulating LSFB processes, the particles and the liquid exit the fluidization column and re-enter as the cycle is completed (Kunii \& Levenspiel, 1991). However, Semi-batch LSFB processes require that only the liquid circulates into and out of the cylindrical column, while the solid particles remain confined within the column. The choice of the type of LSFB is process specific; their use is equally prevalent in industrial processes, however, the focus of this study is on semi-batch LSFBs.

A typical semi-batch liquid fluidized bed process can be seen in Figure 1. The liquid is pumped from its storage tank through the bottom of the fluidization column where it enters a "calming section". The calming section is often installed before the distributor to ensure a uniform velocity profile (velocity in the upwards direction is uniform across the length of the distributor), this prevents bulk movement in certain sections of the column (Epstein, 2003). The liquid then leaves the top of the column back into the storage tank where it is recycled into the fluidized bed. 


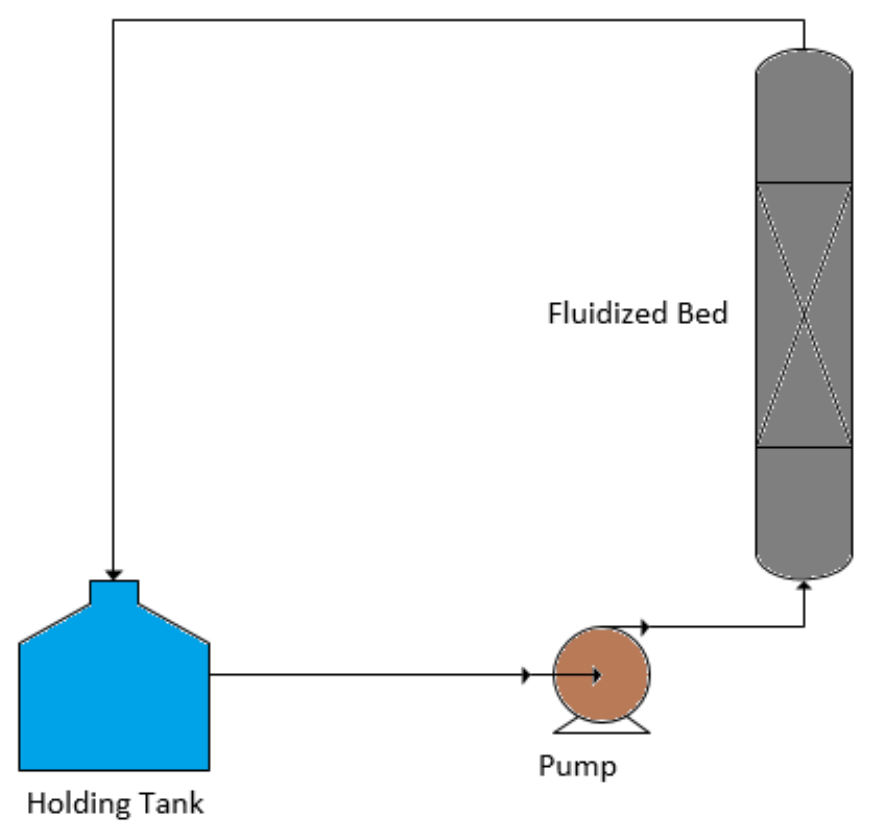

Figure 1: Semi-batch liquid-particle fluidized bed.

Minimum fluidization occurs when the fluid velocity is increased to a value that transforms the bed of particles from its static to its dynamic form. This value is conventionally reported as the "minimum fluidization velocity", $U_{m f}$. Fluidized beds are usually run at multiple times the minimum fluidization velocity (i.e. $1.3 \times U_{m f}$ ) (Kunii \& Levenspiel, 1991). Theoretically, $U_{m f}$ for the idealized case of hard, non-porous particles with constant shape, size, and density, with known minimum fluidization porosity, $\varepsilon_{m f}$, can be calculated by the widely accepted Ergun Equation (Ergun, 1952):

$$
\frac{-d p_{f}}{d z}=\frac{150 U_{m f} \mu_{f}\left(1-\varepsilon_{m f}\right)^{2}}{\varphi_{p}^{2} d_{p}^{2} \varepsilon_{m f}^{3}}+\frac{1.75 U_{m f}^{2} \rho_{f}\left(1-\varepsilon_{m f}\right)}{\varphi_{p} d_{p} \varepsilon_{m f}^{3}}
$$

where, $\frac{-d p_{f}}{d z}$ is the frictional pressure gradient across the column, $d_{p}$ is the diameter of the particle, $\varphi_{p}$ is the particle sphericity, $\rho_{f}$ is the liquid density, and $\mu_{f}$ is the liquid viscosity. If $\varepsilon_{m f}$ is not known, the following correlation can be used, and is valid for $0.2<\varphi_{p} \leq 1$, and $d_{p}>0.05 \mathrm{~mm}$ (Lucas et al., 1986):

$$
\varepsilon_{m f}=\frac{0.415}{\varphi_{p}^{0.483}}
$$


Ergun's equation can be further simplified by using the definitions for Reynolds and Archimedes (Ar) numbers, and reduces to (Epstein, 2003):

$$
\begin{gathered}
R e_{m f}=\frac{d_{p} U_{m f} \rho_{f}}{\mu_{f}} \\
A r=\frac{g d_{p}^{3} \rho_{f}\left(\rho_{p}-\rho_{f}\right)}{\mu_{f}^{2}} \\
R e_{m f}=\left(C_{1}^{2}+C_{2} A r\right)^{0.5}-C_{1} \\
C_{1}=\frac{150\left(1-\varepsilon_{m f}\right)}{1.75 \varphi_{p}}=\frac{42.86\left(1-\varepsilon_{m f}\right)}{\varphi_{p}} \\
C_{2}=\frac{\varphi_{p} \varepsilon_{m f}^{3}}{1.75}=0.5714 \varphi_{p} \varepsilon_{m f}^{3}
\end{gathered}
$$

where $R e_{m f}$ is the minimum fluidization Reynolds number, $\rho_{p}$ is the particle density, and $\boldsymbol{g}$ is the acceleration due to gravity. Thus, it is possible to theoretically calculate the minimum fluidization velocity by knowing $d_{p}, \rho_{f}, \rho_{p}, \mu_{f}$ and $\varepsilon_{m f}$.

For systems other than mono-sized smooth spheres, extensive research has been done where the values for $C_{1}$ and $C_{2}$ were determined. A summary of $C_{1}$ and $C_{2}$ values can be found in Epstein (2003).

\subsection{Experimental Methods}

The success of a computer simulation relies on how accurately it resembles reality. Thus, researchers dealing with modelling and simulation often validate their result with experimental data. In conducting experiments, one must identify which important factors are to be tested, and thus decide on an experimental method. Experimental methods can be broadly classified as intrusive or non-intrusive. Intrusive methods usually require a probe to be placed inside of an experimental setup, usually in contact with the system components (Werther, 1999). Alternatively, non-intrusive experimental methods are set up outside of the geometry and thus do not interfere with the flow (Sun \& Yan, 2016). Intrusive methods are very problematic for liquid-solid fluidized beds because they might disturb liquid and particle flows. Intrusive methods are usually used in industry where the geometry might have a diameter of one to several meters, and where non- 
intrusive methods cannot give quality experimental data. The reader is referred to Werther (1999) for a comprehensive review of intrusive experimental methods. The focus of this study, however, is on non-intrusive experimental methods.

Non-intrusive experimental methods are clearly the preferred option because as previously mentioned, they do not interfere with liquid-fluidized bed flow (both liquid and particle). There are many non-intrusive experimental methods used to obtain experimental data for LSFBs and some include: Particle Image Velocimetry (PIV) (Peng et al., 2014, 2016), Radioactive Particle Tracking (RPT) (Limtrakul et al., 2005), Positron Emission Particle Tracking (PEPT) (Sun \& Yan, 2016), Laser Doppler Anemometry/Phase Doppler Anemometry (LDA/PDA), Electrical Capacitance Tomography (ECT), and Electrical Resistance Tomography (ERT) (Sun \& Yan, 2016). A brief explanation will be given on each of these methods, but the focus of this study is on ERT.

\subsubsection{Particle Image Velocimetry}

PIV is a non-intrusive experimental technique that measures particle velocity and solid fluxes. A typical PIV setup includes a charged coupled device (CCD) camera, a light source, a computer with image processing software, and sometimes an electrical motor with a rotating transparency, or a PIV controller are added (Kashyap et al., 2011; Sutkar et al., 2015). PIV measures particle velocity by using the rotating transparency element to measure the distance that a particle travels over a specified time interval, set by the camera. Measuring particle velocity in this manner requires very tedious manual analysis, and becomes challenging when the fluidized beds are dense (Laverman et al., 2008). For dense beds, instead of measuring individual particle velocity, an average velocity is measured over a predefined volume. The problem is that PIV cannot provide the exact number of particles within that specified volume, and a further modification to the image processing software is needed (Sun \& Yan, 2016).

\subsubsection{Radioactive Particle Tracking}

In RPT technique, a radioactive foreign particle, matching in size, shape, and density to the fluidized particles, is added to the fluidized bed. Outside of the bed, a ring of inorganic scintillation detectors is placed $40-60 \mathrm{~mm}$ away from the surface of the bed. The radioactive particle emits gamma rays which are captured by the detectors which give the radioactive particle position. The particle is tracked over several hours and the time integration of these positions provide local 
particle velocities which can be combined to form an averaged velocity field. From the average velocity field, it is possible to obtain fluidized particle trajectories and velocities (Chaouki et al., 1997). The downside of RPT is that it is very time consuming as the experiment must run for several hours undisturbed. Also, the calibration of RPT is tedious because the tracer location must be known for hundreds and sometimes thousands of iterations (Sun \& Yan, 2016). Finally, the tracer requires a nuclear reactor to activate and there is the inherent risk while working with radioactive material, especially with beta radiation, which is used in RPT.

\subsubsection{Positron Emission Particle Tracking}

PEPT uses similar concept to RPT above, but uses a tracer that emits a positron. A positron is instantly destroyed when there is an electron in its vicinity, producing two gamma rays. These gamma rays are produced back to back and are detected by two position-sensitive detectors. The detectors absorb the gamma rays and the position (and trajectory) of the particle becomes known because the particle must be in a straight line connecting the two gamma rays (Parker et al., 1997). Once the tracer positions are known, the average velocity field can be obtained as mentioned in section 2.2.2. PEPT can thus provide other hydrodynamic properties such as: particle residence time distribution (RTD) and flow structure characterization. The advantage that PEPT has over RPT is that the activation of the tracer does not require a nuclear reactor, rather it is activated by ion exchange; also, calibration is not required. PEPT supplies detailed information for both dense and dilute fluidized beds, unlike RPT which struggles with dense fluidized beds. The downside of PEPT is it only allows tracking of one particle at a time, which takes an extremely long time, and makes it unsuitable for real-time measurement (Sun \& Yan, 2016).

\subsubsection{Laser Doppler Anemometry / Phase Doppler Anemometry}

LDA and PDA are experimental methods used to measure particle velocity, particle size (only PDA), and particle concentration. LDA and PDA follow what is known as the 'Doppler Effect' which states that, there is a change in the frequency of a wave as the wave source and wave receiver move relative to one another. The LDA/PDA system is usually made up of a laser source, a beam splitter, transmitting and receiving optics, light detectors, a signal processor, an oscilloscope, and a computer (Mathiesen et al., 2000). In LDA and PDA the laser source supplies a laser beam which is directed towards a beam splitter where the beam is split into two waves of different frequencies. The two resultant beams are focused into the measurement volume. When particles pass through 
this volume, the laser beams are scattered and then collected by multiple detectors which produce a signal. Particle velocity is measured by the Doppler frequency and fringe distance (distance between the two beams on the measurement volume) (Kiared et al., 1997). The major advantage of LDA and PDA is their high resolution and fast response, but the downside is they are only applicable to dilute flow systems.

\subsubsection{Electrical Resistance Tomography}

ERT is usually used to measure the solids concentration in a LSFB. Its main advantage over other non-intrusive methods is its ability to provide particle concentration visualization over a specific number of planes. ERT is made up of a sensor element, a data acquisition system (DAS), and a PC control. The sensor is comprised of a ring of electrodes which makes up a plane, and is mounted on the outside perimeter of the fluidized bed. A typical fluidized bed with ERT setup will include multiple planes of sensors. Connected to the sensors is the DAS system. The DAS system injects current into a set of (usually 2) electrodes present within a ring, and collects voltage information from the rest of the electrodes. The rest of the electrodes may have varying voltages depending on the positioning of the fluidized particles within the plane of electrodes. The presence of the particles in a plane of electrodes causes resistance to the current flow, and hence the name electrical resistance tomography (Sharifi \& Young, 2013).

Once the first set of voltage data is collected, the DAS applies an equal current to the next set of electrodes within the ring. To choose which set of electrodes to supply current to next, one must adopt one of four data collecting strategies: adjacent, opposite, diagonal, and conducting boundary strategies (Mann et al., 1997). This is simultaneously done on other planes (electrode rings) as well. The voltage data acquired from the sensors are finally sent to the PC control where an image reconstruction software is used to present $2 \mathrm{D}$ images of the planes. The $2 \mathrm{D}$ representations from each plane may be stacked together and a 3D representation of the fluidized bed may then be constructed (Pakzad et al., 2008). The following subsections will discuss the various system components of ERT: the sensors, the DAS, and image reconstruction. The four data collecting strategies will also be discussed.

\subsubsection{Sensors}

Sensor design is crucial to obtaining accurate data in ERT. The electrodes must be equidistantly distributed around the fluidized bed and must be in continuous contact with the fluid. The number 
of electrodes results in a tradeoff between image resolution and system complexity. The higher the number of electrodes, the higher the spatial resolution, but it also increases the requirements of hardware measurements, and thus influences the real-time performance of the ERT system (Dong et al., 2012). The most common configuration is 16 equally spaced electrodes, made up of gold, silver, platinum, stainless steel or brass. In general, the electrodes must have good conductivity (more conductive than the fluid) and low resistance, low cost, and ease of installation (Scott, 2005).

The size of the electrodes is another important factor in the design of the sensors. It was previously mentioned that the sensors are used to both inject current and collect voltage measurements. Ideally, for injection of current, it is recommended that the electrodes have a large surface area to ensure an even and strong enough current is generated within the fluidized bed. For data collection, however, the electrodes are recommended to have a very small surface area, a needle point would be ideal, to avoid averaging of voltages (Aw et al., 2014). This approach makes the ERT setup very complicated as twice the wiring is needed. For simplicity, usually an optimized, mono-sized electrode is used for both injection and data collection in the design of sensors in ERT. Finally, the length of the cables connecting the electrodes to the DAS must be acceptable. Longer cables may cause current leakage which may lead to undesirable phase-shifted signals (Mann et al., 1997).

\subsubsection{Data Acquisition System}

As previously mentioned, the DAS supplies current to a pair of electrodes and receives the corresponding voltage data values from the rest of the electrodes in the array. It sends the data to the image reconstruction software where $2 \mathrm{D}$ and $3 \mathrm{D}$ representation of the conductivity fields are presented. The DAS is made up of signal sources, an electrode multiplexer array, voltmeters, signal demodulators, and a system controller (Mann et al., 1997). The signal sources include a digital signal generator. The digital signals are then converted to analog signals by a digital to analog convertor (DAC). The DAS also includes a voltage generator which is converted to current by a voltage-to-current convertor. Multiplexers are needed to connect the current source to the pair of electrodes. Furthermore, multiplexers are also needed to obtain the voltage measurements from the rest of the electrodes in the same array (Movafagh et al., 2016).

\subsubsection{Data collecting Strategies}

After the current is supplied to the first pair of electrodes and the voltage measurements from the rest of the electrodes are obtained, the next pair of electrodes need to be selected to supply the 
current to; this requires a data collecting strategy. There are four strategies available in literature: The adjacent, opposite, diagonal, and conducting boundary strategies.

\section{1) Adjacent Strategy}

The adjacent strategy is the most common data collecting strategy in ERT due to minimal hardware requirements and fast image reconstruction (Sharifi \& Young, 2013). In this strategy, the current is supplied to neighboring pair of electrodes and the voltage differences are measured from the rest of the electrodes in the array. The second iteration selects the next adjacent pairs until all pairs have had current flown through them. The total possible independent measurements $(M)$ in this strategy is given by:

$$
M=\frac{N(N-3)}{2}
$$

Where $\mathrm{N}$ is the number of electrodes. For an ERT system with 16 electrodes in one ring, the total number of measurements is 104 . Because the current is supplied to one side of the ring at a time, the current density in the middle of the fluidized bed is very low, and thus the ERT system is very sensitive to noise (Movafagh et al., 2016) in this strategy.

\section{2) Opposite Strategy}

In the opposite strategy, the current is supplied to electrodes that are diametrically opposite to one another. Therefore, on a clock system, 6 o'clock and 12 o'clock positions would be diametrically opposite. The electrode adjacent to the current-injecting electrode is considered as the voltage reference electrode, and all other voltages are measured relative to the reference. The following iterations are done in clockwise manner, and the total number of measurements are given by the following equation (Viergever, 1988):

$$
M=\frac{N}{4}\left(\frac{3 N}{2}-1\right)
$$

Because the current runs through the center of the geometry, the opposite strategy is less sensitive to changes at the boundary, however, the opposite strategy results in lower resolution when compared to the adjacent strategy (Viergever, 1988).

\section{3) Diagonal Strategy}


To explain the diagonal strategy, the reader is referred to Figure 2. This figure shows an array of 16 electrodes, numbered 1 through 16. The first run in the diagonal strategy selects electrode 1 and 2 as the reference current and the reference voltage electrodes, respectively. The current is supplied to odd electrodes not including the reference electrodes: $3,5,7$, $9, \ldots, 15$. The voltage measurements are taken on even nodes, not including the reference: $4,6,8,10, \ldots, 16$. The next iteration, the current and voltage electrodes are changed to electrodes 4 and 3, respectively, and current is supplied to all even electrodes except the reference: $2,6,8,10, \ldots, 16$. While the voltage readings are read from the odd electrodes $1,5,7,9, \ldots, 15$. Because the current goes through the center of the fluidized bed, and due to the developed current field, this strategy provides better quality images and good sensitivity over the whole plane (Hua et al., 1993).

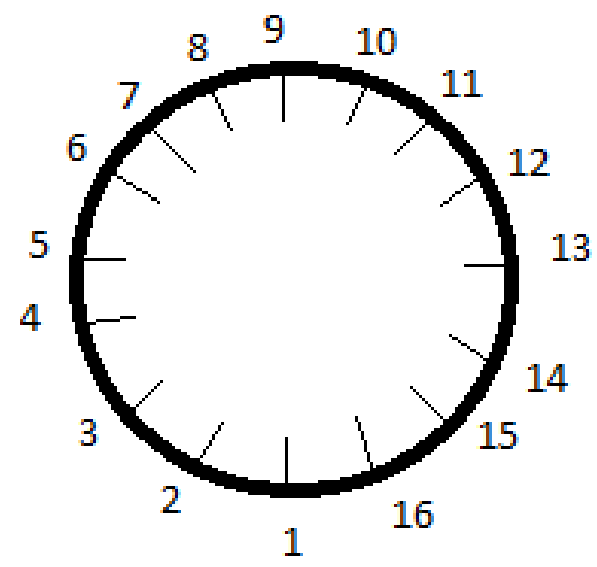

Figure 2: ERT array with 16 electrodes

\section{4) Conducting Boundary Strategy}

The conducting boundary strategy is most suitable for vessels with high electrical conductivity. In this strategy, the current is supplied to each electrode sequentially, while the grounded vessel acts as the current sink. The voltage measurements are referenced to the same earth potential of the conducting boundary (Pakzad et al., 2008). The conducting boundary strategy was developed to counter a problem faced by the adjacent strategy whenever an electrically conductive vessel is employed. Since the current is supplied on one side of the vessel at a time using the adjacent strategy, employing a highly conductive vessel results in the loss of most of the supplied current as it is grounded through the vessel's wall. 


\subsubsection{Image Reconstruction Software}

The voltage data supplied to the DAS are communicated to the host image reconstruction computer. The computer possesses an image reconstruction software that processes the voltage values and presents a resistance distribution within the fluidized bed (Mann et al., 1997). This is done by an image reconstruction algorithm, which can be iterative or non-iterative. The Iterative image reconstruction algorithm is far too slow for image reconstruction because of its high computational requirement. The non-iterative image reconstruction algorithm is often called "linear back projection", and is used to convert voltage values obtained from the electrodes into conductivity values to present a 2D image of the plane (Barber et al., 1983). The images obtained from individual arrays of electrodes can then be stacked to obtain a 3D image of the fluidized bed.

\subsubsection{Electrical Capacitance Tomography}

ECT is one of many non-intrusive experimental methods that use tomography, others include: $\mathrm{x}$ ray computed tomography (x-ray CT), gamma-ray computed tomography (gamma-ray CT), and ERT (Sun \& Yan, 2016). ECT is very similar to ERT in terms of experimental set-up, and the main difference is the fluid used. In ERT, the fluid used must be conductive, while in ECT the fluid used must be non-conductive (air for example) (Pakzad et al., 2008). In ECT, a ring of electrodes is mounted on the side of the fluidized bed. A current is supplied to a pair of electrodes, consecutively, and the capacitance between the excited electrodes and the rest are measured. The capacitance will change depending on the concentration of the solid particles as they fluidize in the bed. The idea is that ECT will use the capacitance differences from all sets of electrodes to reconstruct a 2-D image of solids concentration distribution within the plane. Usually 2 or more electrode arrays are used, and 2D images from each plane are stacked to obtain a 3-D representation of the fluidized bed (Porzuczek, 2014). The quality of the image can be improved by increasing the number of electrodes, and generally, for good quality images, the number of electrodes must exceed 12 (Peng et al., 2012). Another technique related to ECT is ECVT (V stands for volume) which allows the reconstruction of 3D images directly. The problem with ECT is that the change of capacitance in one location will affect the rest of the domain, and thus will affect the resultant reconstruction image. Therefore, ECT is highly sensitive to errors (Sun \& Yan, 2016). 


\subsubsection{Concluding Remarks}

This concludes the literature review of the various experimental methods employed to investigate LSFBs. As was seen, each of the reviewed experimental methods possess advantages and disadvantages, however, the ERT experimental method was selected for validation of simulations in this study. This choice will be discussed towards the end of the chapter; however, the main reason was due to the scarce use of this experimental method in the applications of CFD-DEM simulations of LSFBs. The next section introduces the numerical approach and highlights its importance to LSFB design, while focusing on CFD, DEM, and the CFD-DEM coupling simulation methods.

\subsection{The Numerical Approach}

There are many numerical approaches to the simulation of two-phase systems. The simulation of two-phase systems is done by modeling each phase separately, followed by their coupling to form a complete representation of the system. There are two approaches to the modelling of each phase: the continuum approach and the discrete approach (Van der Hoef et al., 2004, 2008). The decision behind the choice of approach for both liquid and solid phases will be discussed in further detail below, however, the approach that will be used in this study is the coupled continuum (for liquid) and discrete (for solid) approach; more specifically, the CFD-DEM approach.

The largest operational LSFBs are in the scale of several meters, however, the behavior of these beds is governed by particle-particle collisions and particle-liquid interactions, which happen in much smaller scales. This "large separation of scales" is the reason why there exists a number of approaches to the simulation of LSFBs (Van der Hoef et al., 2006, 2008). One approach is to model the system on a macroscopic level, and decide that although the solid and liquid phases must be modelled separately, both phases can be considered as two fluids. As such, this approach is called the two-fluids model (TFM). In the TFM, the two phases are considered as separate continua which exchange momentum. The TFM is computationally convenient, but its downside is that it cannot account for particle-particle collisions (Anderson \& Jackson, 1967; Gidaspow, 1994; Zhu et al., 2007).

Another approach is to treat both the liquid and the solid phases as discrete entities, and that the solid particles collide with the smaller liquid molecules. This essentially results in an extremely accurate description/simulation of the liquid-solid fluidization phenomenon (Cook et al., 2004). 
Some research groups investigated this discrete-discrete approach, including the Smoothed Particle Hydrodynamics-DEM (SPH-DEM) (Robinson et al., 2014) and pseudo-particle methodDEM (PPM-DEM) (Ge \& Li, 2003), among others. The problem with discrete-discrete approaches is that they are very computationally demanding, as the equation of motion for every solid particle must be solved in parallel with the discretized fluid phase (Robinson et al., 2014).

The third approach is to model the fluid as a continuum and the solid particles as discrete entities. This approach is more computationally demanding than the TFM approach, but much less than the discrete-discrete approach. Alternatively, modelling the particles as discrete entities provides an adequate level of detail (particle-particle collisions, and particle-fluid interaction) that is not found in TFM. Some continuum-discrete approaches include: Direct numerical simulation (DNS)-DEM (Hu, 1996; Pan et al., 2002), Large Eddie Simulation (LES)-DEM (Zhou et al., 2004), LatticeBoltzmann-DEM (Van der Hoef et al., 2006), in addition to the CFD-DEM model. The CFD-DEM model is preferred for its superior computational convenience (Zhu et al., 2007), and thus was chosen to be the focus of this study.

\subsubsection{Discrete Element Method: Governing Equations}

The discrete element method models the motion of individual particles separately (Cundall \& Strack, 1979). There are two approaches to DEM that are most common in literature: the soft sphere approach and the hard sphere approach. The soft sphere approach assumes that colliding particles overlap at the contact points instead of deforming (Kafui et al., 2002). An advantage to the soft sphere approach is that it is capable of handling multiple particle contacts simultaneously, which is a necessity for liquid-particle fluidization. Alternatively, in the hard sphere approach, a sequence of collisions is analyzed one collision at a time (Luding, 2008). With a fluidization system having hundreds of thousands of particles, the hard sphere approach is simply not feasible. Thus, the focus of this research study is on the soft sphere model.

A particle in granular flow has both translational and rotational motion. The DEM governing equations are (Van der Hoef et al., 2006):

$$
m_{i} \frac{d \boldsymbol{v}_{\boldsymbol{i}}}{d t}=\sum_{j} \boldsymbol{F}_{i j}^{c}+\boldsymbol{f}_{i}^{f}+\boldsymbol{F}_{i}^{g}
$$




$$
I_{i} \frac{d \boldsymbol{\omega}_{\boldsymbol{i}}}{d t}=\sum_{j}\left(\boldsymbol{M}_{i j}^{t}+\boldsymbol{M}_{i j}^{r}\right)
$$

where, $m_{i}$ is the mass of particle i, $\boldsymbol{v}_{\boldsymbol{i}}$, is the velocity of particle $\mathrm{i}, \boldsymbol{F}_{i j}^{c}$ is the contact force between

particle $\mathrm{i}$ and particle $\mathrm{j}$ or particle $\mathrm{i}$ and geometry, $\boldsymbol{f}_{i}^{f}$ is the particle-fluid interaction force acting on particle i, $\boldsymbol{F}_{i}^{g}$ is the gravitational force on particle $\mathrm{i}, I_{i}$ is the moment of inertia, $\boldsymbol{\omega}_{\boldsymbol{i}}$ is the angular velocity of particle i, and $\boldsymbol{M}_{i j}^{t}$ and $\boldsymbol{M}_{i j}^{r}$ are the rotational torque and the rolling resistance torque acting on particle $\mathrm{i}$ by particle $\mathrm{j}$, respectively.

The coupling of the CFD and DEM simulation methods is done through the interaction force, $\boldsymbol{f}_{i}^{f}$, and this concept is explained in moderate detail in section 2.3.4.

\subsubsection{DEM: Contact Models}

The discrete element method (soft sphere) makes certain essential assumptions about how particles collide and interact with one another (Cundall \& Strack, 1979):

1) Instead of collisions, the particles are allowed to (slightly) overlap. This overlap is much smaller than the particle diameter, and is representative of an elastic collision.

2) Plastic deformations of individual particles can be ignored, because the deformations are assumed to be very small compared to the deformation of the flowing granular system.

Many contact models have been proposed, however, contact models can be classified as: linear and non-linear. The linear contact models are more intuitive and simplistic, and the most common linear contact model is the Cundall \& Strack (1979) Spring Dash-pot Model. Alternatively, the more complex non-linear model was initially proposed by Hertz (1882) and the Hertz-Mindlin \& Deresiewicz (1953) model that followed.

Contradictions exist in literature from various sources as to which of the two models (linear or non-linear) provides a more accurate description of how particles come in contact. As an example, Di Renzo \& Di Maio (2004) conducted investigations which proved that in certain cases, linear models produce better results. Zhu et al. (2007) explains that theoretically, the non-linear models should present better results but explains that theoretical models are based on perfect geometry of particles, where in reality this is not the case. Nonetheless, the Hertz-Mindlin \& Deresiewicz contact model was applied in this research, and is given as such (DEMSolutions, 2010): 


$$
\begin{gathered}
\boldsymbol{F}_{i j}^{n}=-\frac{4}{3} Y^{*}\left(\delta_{n}\right)^{\frac{3}{2}} \sqrt{R^{*}}-2 \gamma \sqrt{\frac{5 S_{n} m^{*} V_{n}^{r e l}}{6}} \\
S_{n}=2 Y^{*} \sqrt{R^{*} \delta_{n}} \\
\gamma=\frac{\ln e_{r}}{\sqrt{\ln ^{2} e_{r}+\pi^{2}}} \\
\boldsymbol{F}_{i j}^{t}=-S_{t} \delta_{t}-2 \gamma \sqrt{\frac{5 S_{t} m^{*} V_{t}^{r e l}}{6}} \\
S_{t}=8 G^{*} \sqrt{R^{*} \delta_{t}}
\end{gathered}
$$

where, $\boldsymbol{F}_{i j}^{n}$, and $\boldsymbol{F}_{i j}^{t}$, are the normal and tangential components of the contact force, respectively, $Y^{*}$ is the equivalent Young's modulus, $\delta_{n}$ is the normal overlap, $R^{*}$ is the equivalent radius, $S_{n}$ is the normal stiffness, $m^{*}$ is the equivalent mass, $e_{r}$ is the coefficient of restitution, $S_{t}$ is the tangential stiffness coefficient, $\delta_{t}$ is the tangential overlap, $G^{*}$ is the equivalent shear modulus, and $V_{n}^{r e l}$ and $V_{t}^{\text {rel }}$ are the normal and tangential components of relative velocity, respectively.

\subsubsection{DEM: Torque Models}

As two particles collide, they will generate a torque (eq. 2-11) that has two contributions: rotational torque, and rolling resistance (friction) torque. The rotational torque is caused by inter-particle collisions in the tangential direction, and it causes the particles to rotate. The rolling resistant torque arises from the asymmetric normal traction distributions, and it resists the rotation caused by the rotational torque (Norouzi et al., 2016). The rotational torque, $\boldsymbol{M}_{i j}^{t}$, is defined as:

$$
\boldsymbol{M}_{i j}^{t}=R_{i} \boldsymbol{n}_{i j} \times \boldsymbol{F}_{i j}^{c}
$$

where $R_{i}$ is the radius of particle $\mathrm{i}$, and $\boldsymbol{n}_{i j}$ is the unit vector in the normal direction. There are three main approaches depicted in literature to model rolling resistance torque: Constant torque model (Model 1), Viscous model (Model 2), and Spring-Dashpot Model (Model 3) (Ai et al., 2011). The constant torque model (Modal 1) was applied in this research, and the reader is referred 
to (Norouzi et al., 2016) for an in-depth analysis on torque models. Model 1 is given as (Zhou, 1999):

$$
\begin{gathered}
\boldsymbol{M}_{i j}^{r}=-\mu_{r} R_{e f f}\left|\boldsymbol{F}_{i j}^{n}\right| \Psi_{i j} \\
\Psi_{i j}=\frac{\psi_{i}-\psi_{i}}{\left|\psi_{i}-\psi_{i}\right|}
\end{gathered}
$$

where, $\mu_{r}$ is the rolling friction coefficient, $R_{e f f}$ is the effective radius, $\psi_{i j}$ is the unit vector of relative angular velocity, and $\psi_{i}$ and $\Psi_{j}$ are the unit vectors of angular velocities for particles $i$ and $\mathrm{j}$, respectively.

\subsubsection{Computational Fluid Dynamics: Governing Equations}

The fundamental principles of computational fluid dynamics are represented by governing equations. These are the conservation equations of momentum, mass, and energy. This study will deal with systems with no chemical reactions, and no exchange of energy across the boundaries of said systems. Therefore, the energy conservation equation and its discussion is beyond the scope of this study, and only the conservation of mass and momentum will be discussed.

The conservation of mass, or the continuity equation is presented in vector notation, irrespective of the coordinate system as (Chung, 2009; (Bird et al., 2002):

$$
\frac{\partial \rho_{f}}{\partial t}+\left(\nabla \cdot \rho_{f} \boldsymbol{u}\right)=0
$$

For Newtonian and incompressible fluids, the conservation of momentum equations reduce to the Navier-Stokes Equations (Bird et al., 2002):

$$
\rho_{f} \frac{D \boldsymbol{u}}{D t}=(-\nabla p)+\mu_{f} \nabla^{2} \boldsymbol{u}+\rho_{f} \boldsymbol{g}
$$

The solution to the Navier-Stokes equations requires a clear definition of initial and boundary conditions, that is, how the fluid interacts with the system's boundaries, and what are the initial conditions of the fluid. In fluid mechanics, there are a number of boundary conditions which are commonly applied. These boundary conditions are abundant in literature (Bird et al., 2002; Çengel \& Cimbala, 2006; Morrison, 2001). 
The Navier-Stokes equations represent an exact description of the motion of an incompressible, Newtonian fluid. These equations cannot be solved analytically unless fluid motion is simplified by making a number of assumptions. The problem is if too many assumptions are made, the analytical solution may vary considerably from reality, which renders the solution invalid. Alternatively, the Navier-Stokes equations can be solved through numerical approach. One numerical approach that solves for the Navier-Stokes equations is Computational Fluid Dynamics (CFD). More specifically, CFD uses discretization techniques to obtain an approximation to the solution of the Navier-Stokes equations (Anderson \& Wendt, 1995). Discretization techniques transform the non-linear Navier-Stokes equations into a number of algebraic equations which can be solved by numerical methods and iterative computer programs (Versteeg \& Malalasekera, 1995).

Before discretization techniques can be discussed, it is important to briefly review common CFD software currently applied in industry and research. CFD codes can either be commercial or open source. Some commercial codes include: ANSYS Fluent, ANSYS CFX, COMSOL, PHOENICS, and FLOW3D, while some open source CFD codes include: OpenFOAM, SU2, and TYPHON. The advantage of open source software is their accessibility and the ability to run parallel simulations, but usually may not include a user-friendly user interfaces (UIs). However, commercial software often aim for the "full package" and include a user friendly UI, a solver, pre and post processing, CAD-integration, and so on, but acquiring licenses can be very expensive (Tu et al., 2013).

The commercial software used in this study (ANSYS-Fluent) applies the finite volume method (FVM) for discretization. The FVM splits the geometry into multiple finite volumes, commonly known as 'cells'. These cells can be triangular and quadrilateral (2D), or tetrahedral and hexahedra (3D), or any of their combination, and the conservation equations are solved on discrete locations within these cells, usually at the center. The main advantage of FVM is that it produces meshes that can handle complex geometries; this is why FVM is the most used discretization method in CFD codes, both open source and commercial (Chung, 2009; J. Ge et al., 2015; Tu et al., 2013). The remainder of this CFD section will discuss mesh and geometry generation, obtaining and analyzing CFD solutions, and turbulence models found in CFD applications. 
There are multiple computer programs with simple UIs that allow the creation of geometry using computer-aided-design (CAD) drawings including SOLIDWORKS, ANSYS Workbench, and many more. After the geometry is defined, the next step is to generate a mesh. The CFD solution is critically dependent on the mesh density, and how this density varies along the geometry is directly related the quality and accuracy of a given solution. An improperly designed mesh can easily lead to divergence from the expected solution and high instability (D. C. Barber et al., 2007). A mesh can be either structured or unstructured. Unstructured meshes generally require higher number of cells, and need longer computational times. There is no consensus in literature however, as to whether unstructured meshes produce better results, and results generally vary from one application to the other. As an example, De Santis et al. (2010) studied the use of hexahedral structured meshes in CFD modelling of coronary arteries, and showed that the hexahedral structured meshes required 6 times less cells, and 14 times less computational time to produce the same accuracy of results as an unstructured tetrahedral mesh. Therefore, the choice of using structured vs. unstructured meshes is geometry and application specific.

Once the geometry and mesh are created, the next step is to obtain the numerical solution. The numerical solution is obtained by the CFD solver through five steps: initialization, solution control, monitoring solution, CFD calculation, and checking for convergence (Tu et al., 2013). The initialization step requires the input of discrete values of flow properties, such as velocities and pressure, which act as the boundary conditions. The solution control step is where the velocitypressure coupling algorithms are solved by iterative methods. Monitoring the solution, CFD calculation, and checking for convergence steps are interlinked, as the convergence of the iteration step requires constant monitoring of the conservation of flow parameters (Versteeg \& Malalasekera, 1995).

Once the solution is acquired, a visual representation of the numerical solution can be obtained by using the post-solver CFD element. Many CFD codes include in-built GUIs which can present the obtained solution through many visual representations including: X-Y plots, vector plots, and contour plots.

\subsubsection{CFD: Turbulence Modeling}

When the Reynolds number of a fluid exceeds a certain critical value, $R e_{c r i t}$, the flow is said to be turbulent. Almost all industrial applications with fluid flow experience turbulence, and turbulent 
processes are often preferred because they provide superior mixing. Many processes benefit from this quality because heat and mass transfer are hugely magnified, which is especially important in fluidized bed processes (Launder \& Spalding, 1974).

In the turbulent region, the flow is chaotic and unpredictable, and local flow parameters, such as velocity and pressure, fluctuate chaotically over time. Therefore, to account for turbulence in various processes, turbulence models were developed. There are many turbulence models present in literature including: mixing length models, k- $\varepsilon$ model, Reynolds stress equations model, and algebraic stress equations models. The most commonly used model to describe turbulence is the $k-\varepsilon$ model. The $k-\varepsilon$ model solves for the turbulent kinetic energy, $k$, and for the dissipation of turbulent kinetic energy, $\varepsilon$ (Versteeg \& Malalasekera, 1995). The $k-\varepsilon$ model was first introduced by Launder \& Spalding (1974) and is given as follows:

$$
\begin{gathered}
\frac{\partial(\rho k)}{\partial t}+\operatorname{div}(\rho k \boldsymbol{u})=\operatorname{div}\left[\frac{\mu_{t}}{\sigma_{k}} \operatorname{grad} k\right]+2 \mu_{t} \boldsymbol{E}_{i j} . \boldsymbol{E}_{i j}-\rho \varepsilon \\
\frac{\partial(\rho \varepsilon)}{\partial t}+\operatorname{div}(\rho \varepsilon \boldsymbol{u})=\operatorname{div}\left[\frac{\mu_{t}}{\sigma_{\varepsilon}} \operatorname{grad} \varepsilon\right]+C_{1 \varepsilon} \frac{\varepsilon}{k} 2 \mu_{t} \boldsymbol{E}_{i j} \cdot \boldsymbol{E}_{i j}-C_{2 \varepsilon} \rho \frac{\varepsilon^{2}}{k} \\
\mu_{t}=\rho C_{\mu} \frac{k^{2}}{\varepsilon}
\end{gathered}
$$

where $\mu_{t}$ is the turbulent viscosity, $\boldsymbol{E}_{i j}$ is the mean rate of deformation, and $C_{\mu}, C_{1 \varepsilon}, C_{2 \varepsilon}, \sigma_{k}$, and $\sigma_{\varepsilon}$ are adjustable constants. Versteeg \& Malalasekera (1995) reported the standard values for these constants as:

$$
\mathrm{C}_{\mu}=0.09 ; \sigma_{k}=1.00 ; \sigma_{\varepsilon}=1.30 ; C_{1 \varepsilon}=1.44 ; C_{2 \varepsilon}=1.92
$$

This concludes the discussion on CFD. The next subsection discusses how DEM and CFD simulation methods are coupled to provide a complete representation of the LSFB system.

\subsubsection{CFD-DEM Coupling}

In the opening remarks of this chapter, it was stated that there are three approaches used to model liquid-particle flows: Continuum-Continuum approach (TFM), discrete-discrete approach and continuum-discrete approach. It was explained that the continuum-discrete approach, and 
particularly the CFD-DEM approach, provides a superior level of detail relative to TFM, with a considerably less computational time relative to the discrete-discrete approach, and thus CFDDEM approach was chosen to be the topic of this study (Tu et al., 2013; Van der Hoef et al., 2004, 2006, 2008; Zhu et al., 2007). In the CFD-DEM approach, DEM models the solid particles and evaluates the equations of motion of individual particles (see section 2.3.2), while CFD models the liquid phase by solving the locally-averaged Navier-Stokes equations.

There exists two volume (locally)-averaged Navier-stokes models in literature, Gidaspow (1994) simply called them Model A and Model B. In this research, Model A was chosen, and the reader is referred to Gidaspow (1994) for further explanation. Model A of the locally averaged NavierStokes equations is given as:

$$
\begin{gathered}
\rho_{f} \varepsilon_{f}\left(\frac{\partial \overline{\boldsymbol{u}}}{\partial t}+\nabla \cdot(\overline{\boldsymbol{u}} \overline{\boldsymbol{u}})\right)=-\varepsilon_{f} \nabla p-\boldsymbol{F}^{\boldsymbol{f}}+\nabla \cdot\left(\varepsilon_{f} \boldsymbol{\tau}\right)+\rho_{f} \varepsilon_{f} \boldsymbol{g} \\
\frac{\partial \varepsilon_{f}}{\partial t}+\left(\nabla \cdot \varepsilon_{f} \overline{\boldsymbol{u}}\right)=0
\end{gathered}
$$

where $\overline{\boldsymbol{u}}$ is the volume-averaged liquid velocity, $\boldsymbol{F}^{\boldsymbol{f}}$ is the fluid-particle interaction force in a cell, and $\boldsymbol{\tau}$ is the volume-average stress tensor. As previously mentioned in section 2.3.2, the coupling

is done through the interaction force. Noting that the particle-fluid interaction force $\boldsymbol{f}_{i}^{f}$ is positive in eq. (2-10) (for DEM), and negative in eq. (2-25) (for CFD), this satisfies Newton's third law. However, before the connection between $\boldsymbol{F}^{\boldsymbol{f}}$ (fluid-particle interaction force in a cell, CFD) and $\boldsymbol{f}_{i}^{f}$ (particle-fluid interaction force) is established, it is important to discuss how this force is formulated.

\subsubsection{Fluid-Particle Interaction Forces}

The fluid-particle interaction force is a combination of multiple forces: drag force, Basset force, virtual mass force, lift forces, and the pressure gradient force (which includes buoyancy force) (Zhu et al., 2007). As briefly mentioned in the opening remarks of section 2.1, the interaction forces are forces that act on the particles as the liquid is pumped through the fluidization system. The particle-fluid interaction force, $\boldsymbol{f}_{i}^{f}$, can be written in equation form as such:

$$
\sum \boldsymbol{f}_{i}^{f}=\boldsymbol{F}_{\text {Basset }}+\boldsymbol{F}_{V m}+\boldsymbol{F}_{\text {Saff }}+\boldsymbol{F}_{M a g}+\boldsymbol{F}_{p}+\boldsymbol{F}_{d}
$$


where $\boldsymbol{F}_{\text {Basset }}$ is the Basset force, $\boldsymbol{F}_{p}$ is the pressure gradient force, $\boldsymbol{F}_{V m}$ is the virtual mass force, $\boldsymbol{F}_{\text {Saff }}$ and $\boldsymbol{F}_{\text {Mag }}$ are Saffman and Magnus lift forces, respectively, and $\boldsymbol{F}_{d}$ is the drag force.

The Basset force accounts for viscous effects. It describes the time delay that the boundary layer surrounding the particle's surface experiences as the particle changes its relative velocity (MorenoCasas \& Bombardelli, 2016) and given as:

$$
\left.\boldsymbol{F}_{\text {Basset }}=\frac{3}{2} d_{p}^{2} \sqrt{\pi \rho_{f} \mu_{f}}\left[\int_{t_{0}}^{t} \frac{(\boldsymbol{u}-\boldsymbol{v})}{\sqrt{t-t^{\prime}}} d t^{\prime}+\frac{(\boldsymbol{u}-\boldsymbol{v})_{0}}{\sqrt{t}}\right]\right]
$$

where, $\boldsymbol{u}$ is the fluid superficial velocity, $\boldsymbol{v}$ is the particle translational velocity, $t_{0}$ is the initial time, $t$ is at any time, and $(\boldsymbol{u}-\boldsymbol{v})_{0}$ is the initial velocity difference.

The virtual mass force is often called "apparent mass force". It occurs when particles accelerate within a fluid, and its effect is similar to adding mass to the particles (Moreno-Casas \& Bombardelli, 2016). The virtual mass is given as (Odar \& Hamilton, 1964):

$$
\begin{aligned}
\boldsymbol{F}_{V m} & =C_{V m} V_{P} \rho_{f} \frac{(\dot{\boldsymbol{u}}-\dot{\boldsymbol{v}})}{2} \\
C_{V m} & =2.1-\frac{0.132}{\left(0.12+A_{c}^{2}\right)} \\
A_{C} & =\frac{(\boldsymbol{u}-\boldsymbol{v})^{2}}{d_{p} \frac{d(\boldsymbol{u}-\boldsymbol{v})}{d t}}
\end{aligned}
$$

where $C_{V m}$ is the virtual mass coefficient, $V_{P}$ is the volume of particle, $\dot{\boldsymbol{u}}$ and $\dot{\boldsymbol{v}}$ are particle and fluid acceleration, respectively.

Lift forces occur due to the rotation of particles, and are divided into two forces: Saffman and Magnus. The Saffman force is due to the pressure distribution caused by the velocity gradient, and Magnus force occurs due to the pressure differential present on the surface of the particle as it rotates. The Lift forces are given as (Rubinow \& Keller, 1961; Saffman, 1965; Zhu et al., 2007):

$$
\begin{gathered}
\boldsymbol{F}_{\text {Saff }}=1.61 d_{p}^{2}\left(\rho_{f} \mu_{f}\right)^{0.5}\left|\boldsymbol{\omega}_{\boldsymbol{c}}\right|^{-0.5}\left[(\boldsymbol{u}-\boldsymbol{v}) \times \boldsymbol{\omega}_{\boldsymbol{c}}\right] \\
\boldsymbol{\omega}_{\boldsymbol{c}}=\nabla \times \boldsymbol{u}
\end{gathered}
$$




$$
\boldsymbol{F}_{M a g}=\frac{\pi}{8} d_{p}^{2} \rho_{f}\left[\left(\frac{1}{2} \nabla \times \boldsymbol{u}-\boldsymbol{\omega}_{d}\right) \times(\boldsymbol{u}-\boldsymbol{v})\right]
$$

where $\boldsymbol{\omega}_{c}$ is the vorticity, and $\boldsymbol{\omega}_{d}$ is the particle rotation.

As the fluid is pumped through the fluidized bed, it experiences a pressure drop due to friction with particles and wall of the column. This results in a pressure gradient across the height of the column. The pressure gradient force generally includes buoyancy force due to gravity and acceleration pressure in fluid, and is given by (Anderson \& Jackson, 1967; Zhu et al., 2007):

$$
\boldsymbol{F}_{p}=-V_{p} \frac{d p}{d x}=-V_{p}\left(\rho_{f} \boldsymbol{g}+\rho_{f} \frac{\boldsymbol{u} d \boldsymbol{u}}{d x}\right)
$$

Lift, Basset, and Virtual mass forces are called "transient forces", and they usually occur in unsteady flows. If the relative velocity between the fluid and the particle is constant, the flow is steady, and thus the transient forces become negligible (Drew \& Lahey, 1990). Also, the Basset

and virtual mass terms become negligible when $\left(\frac{\rho_{f}}{\rho_{s}}\right) \approx 10^{-3}$ (Zhu et al., 2007). Some sources combine transient forces with the drag force and the combination is called "general drag forces" (Yu et al., 2014).

\subsubsection{Drag Models}

The driving force for fluidization comes mainly from the drag force (Zhu et al., 2007). In literature, there have been many empirical correlations and computer simulation methods developed to determine the particle-fluid drag force. These are commonly known as "drag models". There are many drag models in literature, some based on bed pressure drop or bed expansion experiments, such as the Zaki-Richardson drag model (Couderc, 1985). Other models are based on LatticeBoltzmann computer simulations, such as the Hill Koch Ladd model (Lundberg \& Halvorsen, 2008). The reader is referred to Beetstra et al. (2007) and Van der Hoef et al. (2004, 2006, 2008) for a more detailed discussion on the various drag models currently available. The following drag models: the Schiller and Naumann (Schiller \& Naumann, 1933), the Gidaspow (Gidaspow, 1994), and the Syamlal and O'Brien (Syamlal \& O’Brien, 1989), are presented as examples of common drag models:

The Schiller-Naumann drag model: 


$$
\begin{gathered}
\boldsymbol{F}_{d}=\frac{C_{D} R e}{24} \\
C_{d}= \begin{cases}\frac{24\left(1+0.15 R e_{p}^{0.687}\right)}{R e_{p}} & R e_{p}>1000 \\
0.44 & R e_{p}<1000\end{cases} \\
R e_{p}=\frac{\rho_{f d_{p} \epsilon_{f}|\boldsymbol{u}-\boldsymbol{v}|}}{\mu_{f}}
\end{gathered}
$$

where, $C_{d}$ is the drag coefficient, $R e$ is the fluid Reynolds number, and $R e_{p}$ is the particle Reynolds number.

The Gidaspow drag model is given as:

$$
\begin{aligned}
& \boldsymbol{F}_{d}=\frac{\beta_{p f}(\boldsymbol{u}-\boldsymbol{v})}{\rho_{f}} \\
& \beta_{p f}= \begin{cases}150 \frac{\left(1-\epsilon_{f}\right)^{2} \mu_{f}}{\epsilon_{f}\left(d_{p}\right)^{2}}+1.75\left(\left(1-\epsilon_{f}\right) \frac{\rho_{f}}{d_{p}}|\boldsymbol{u}-\boldsymbol{v}|\right. & \epsilon_{f} \leq 0.8 \\
\frac{3}{4} \frac{C_{d}|\boldsymbol{u}-\boldsymbol{v}| \rho_{f}\left(1-\epsilon_{f}\right)}{d_{p}} \epsilon_{f}^{-2.7} & \epsilon_{f}>0.8\end{cases} \\
& C_{d}= \begin{cases}\frac{24\left(1+0.15 R e_{p}^{0.687}\right)}{R e_{p}} & R e_{p}>1000 \\
0.44 & R e_{p}<1000\end{cases}
\end{aligned}
$$

where $\beta_{p f}$ is the momentum exchange coefficient.

The Syamlal-O’brien drag model is given as:

$$
\begin{gathered}
\beta_{p f}=\frac{3}{4} \frac{\epsilon_{s} \epsilon_{f} \rho_{f}}{\boldsymbol{v}_{\boldsymbol{t}}^{2} d_{p}} C_{D}\left(\frac{R e_{s}}{\boldsymbol{v}_{\boldsymbol{t}}}\right)|\boldsymbol{u}-\boldsymbol{v}| \\
C_{D}=\left(\begin{array}{c}
\left.0.63+\frac{4.8}{\sqrt{\frac{R e_{p}}{\boldsymbol{v}_{\boldsymbol{t}}}}}\right)^{2} \\
\boldsymbol{v}_{\boldsymbol{t}}=0.5\left(A-0.06 R e_{p}+\sqrt{\left(0.06 R e_{p}\right)^{2}+0.12 R e_{p}(2 B-A)+A^{2}}\right)
\end{array}\right.
\end{gathered}
$$




$$
\begin{array}{ccc}
A=\epsilon_{f}^{4.14} & B=0.8 \epsilon_{f}^{4.14} & \epsilon_{f} \leq 0.85 \\
A=\epsilon_{f}^{4.14} & B=\epsilon_{f}^{2.65} & \epsilon_{f}>0.85
\end{array}
$$

where $\boldsymbol{v}_{\boldsymbol{t}}$ is the terminal velocity.

\subsubsection{CFD-DEM Coupling: Coupling Schemes}

Depending on the solid volume fraction in a fluid-particle system, CFD-DEM coupling can be done in one of the following three ways: One-way coupling, two-way coupling, or four-way coupling (Elghobashi, 1991; Van der Hoef et al., 2004, 2006, 2008):

1) One-way coupling: When the solid volume fraction in a fluid-particle system is less than $10^{-6}$, the system is very dilute, and thus the particles have no effect on fluid flow. Alternatively, particle flow depends solely on the fluid flow. The solid volume fraction is so small that particle-particle collisions become negligible. Because the only major force present in the system is the force of fluid acting on particle, this coupling scheme is called 'one-way'. An alternative explanation would be, the transfer of momentum only occurs in one way, from the fluid to the particles.

2) Two-way coupling: As the solid volume fraction increases from $10^{-6}$ to $10^{-3}$, the particles become dense enough to affect fluid flow, but not so dense that particle-particle collisions become prevalent. Therefore, in this case the fluid exerts a force on the particles that is equal and opposite of the force that the particles exert on the fluid (Newton's third law). Because momentum is exchanged from particles to fluid and from fluid to particles, this coupling method is called two-way coupling.

3) Four-way coupling: Four-way coupling is needed for systems where particle-particle collisions cannot be ignored. This is particularly true for systems with solid volume fractions higher than $10^{-3}$. Four-way coupling considers all forces previously discussed: contact forces and particle-fluid interaction forces, and provides the highest level of interaction description between the two phases. This coupling method is called four-way because momentum is transferred from particle to fluid, fluid to particle, and particle to particle. 
A visual representation of one-way, two-way, and four-way coupling can be seen in Figure 3 below:

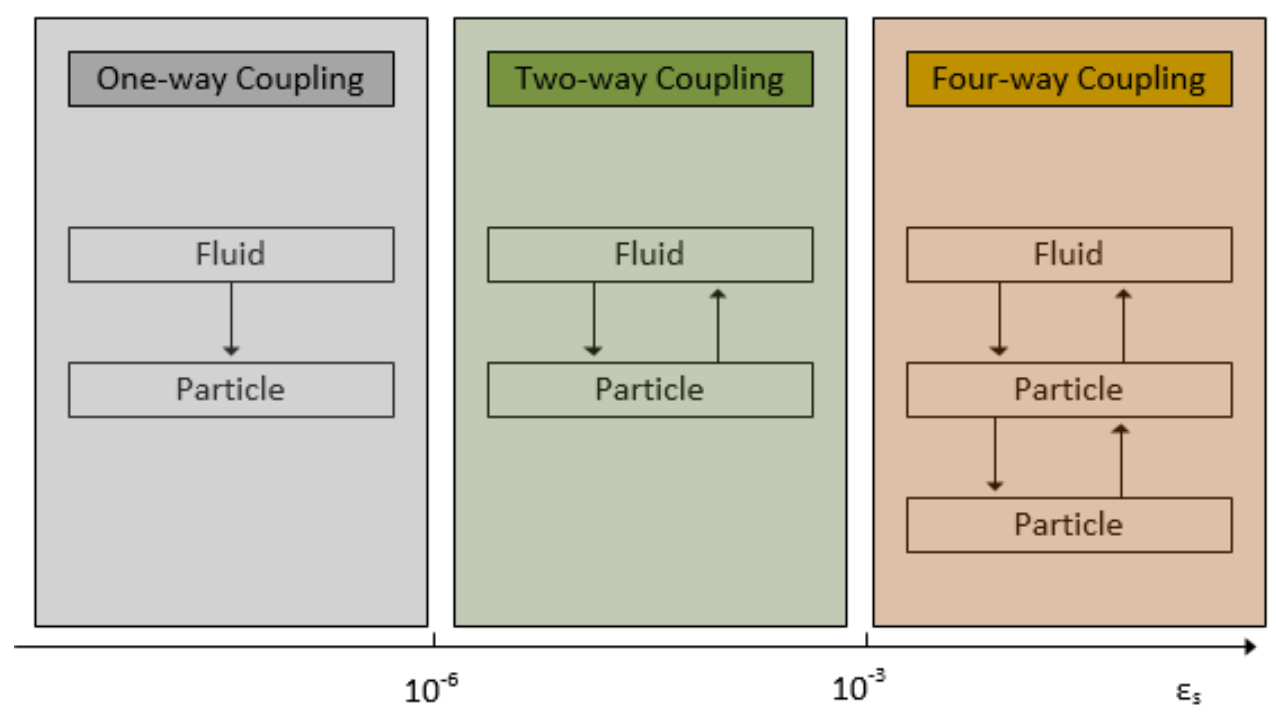

Figure 3: One-way, two-way, and four-way coupling (Van der Hoef et al., 2006).

Coupling between CFD and DEM is only possible due to the particle-fluid interaction forces which can be seen in both the equations of motion for DEM, and the locally averaged Navier-Stokes equations in CFD. There are three coupling schemes that are presented in literature, but only one of which is currently widely accepted. The reader is referred to Zhu et al. (2007) for a detailed discussion on the various coupling scheme, however in this research the coupling scheme presented by $\mathrm{Xu} \& \mathrm{Yu}$ (1997) was employed. This coupling scheme states that at each time step the particle-fluid interaction forces of individual particles present within a computational cell are calculated, by setting initial velocity and position conditions. The sum of the particle-fluid interaction forces acting on the particles is used to obtain the particle-fluid interaction forces acting on the fluid within the same computational cell as such (Hoomans et al., 2000; Jie Li \& Kuipers, 2002; Jintang Li et al., 2004; Xu \& Yu, 1997):

$$
\boldsymbol{F}^{\boldsymbol{f}}=\frac{\sum_{i=1}^{k c} \boldsymbol{f}_{i}^{f}}{\Delta V}
$$

where $\Delta V$ is the volume of the computational cell, and $k_{c}$ is the number of particles present within the computational cell. 
Once $\boldsymbol{F}^{\boldsymbol{f}}$ is known, the relative velocity of fluid and particle can be obtained and used to obtain individual particle-fluid interaction forces of particles for the next time step. A coupling diagram summarizing the coupling scheme is presented in Figure 4 below (Norouzi et al., 2016).

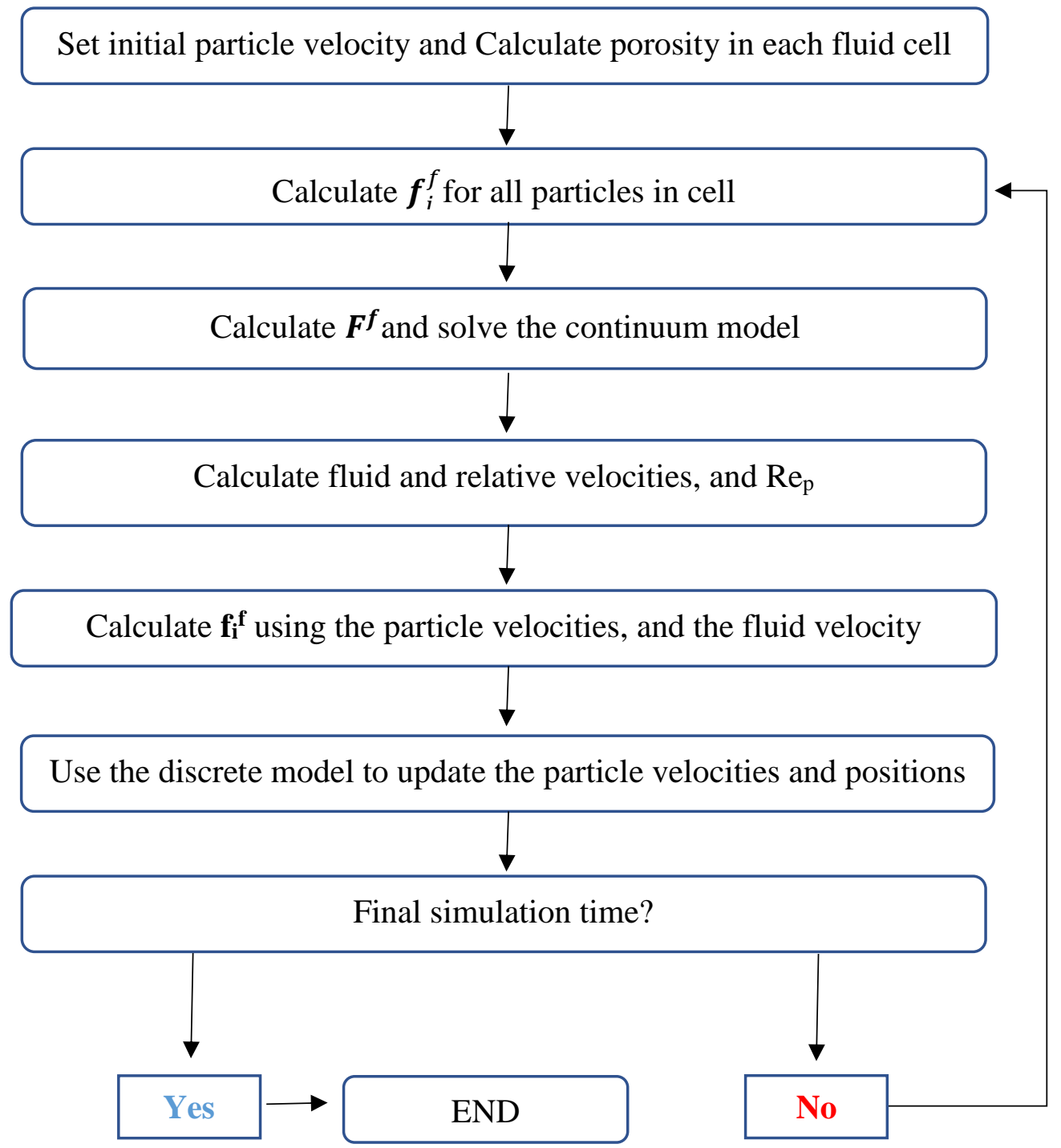

Figure 4: Coupling Diagram (Zhu et al., 2007). 


\subsubsection{Concluding Remarks}

This section introduced various numerical approaches to the simulation of fluid-particle flows: TFM, discrete-discrete, and continuum-discrete approaches. It was explained that CFD-DEM approach is a continuum-discrete approach. The CFD-DEM approach was selected as it provides a compromise between the TFM and discrete-discrete approaches in that it does not require long computational time, while providing adequate level of detail (Zhu et al., 2007). In the CFD-DEM approach, CFD is employed to model the fluid flow by solving the volume averaged Navier-Stokes equations, and DEM is used to model particle flow by solving the equation of motion for each particle. For the purposes of this research, the DEM soft sphere model was chosen, along with the non-linear Hertz-Mindlin contact model. For CFD, Model A was selected as it is pre-implemented by the commercial software used in this research (FLUENT). Finally, the Four-way coupling scheme was selected as it provides useful information about particle-particle contact and particlefluid interaction. The following sections discuss the various statistical models employed in multiphase flow systems, and a stability criterion used to determine the flow regime within a LSFB.

\subsection{Statistical Analysis}

To analyze processes experimentally, statistical design of experiments is often employed. By utilizing statistical methods, the effect of process variables and their interaction on the experimental output can be quantified; this allows for comprehensive process analysis and optimization. One very prominent statistical method is the response surface method (RSM), which employs factorial design of experiments. Many forms of the RSM have been proposed in open literature such as the Central Composite Design (CCD) (Box \& Wilson, 1951), Box-Behnken Design (BBD) (Box \& Behnken, 1960), and D-Optimal Design (H.Myers et al., 2016).

The choice of RSM form ultimately depends on the nature of the design variables and the number of factorial coded levels required. Kazemzadeh et al., (2016) applied the RSM to design ERT experiments and to study the rheological properties of Herschel-Buckley fluids in coaxial mixers. By employing statistical design of experiments, the authors found that the consistency index, the yield stress, and their interaction significantly affect mixing time (experimental output). The use of RSM for process optimization was shown by Pakzad \& Azimi (2017), where the authors employed the RSM for ERT experiments to study the dynamics of particle clouds in stagnant 
water. Therefore, statistically designing experiments can be an extremely valuable tool for process analysis and optimization (Ghafoori et al., 2014; Kazemzadeh et al., 2017b; Kirmizakis et al., 2014; Pakzad et al., 2013; Pakzad \& Azimi, 2017).

\subsection{LSFB Stability}

The hydrodynamics of a LSFB depends greatly on the flow regime which is classified as homogeneous, intermediate (also indeterminate stability, pseudo-homogeneous, or heterogeneous), or bubbling (Foscolo \& Gibilaro, 1987; Gibilaro, 2001; Joshi et al., 2001; Wallis, 1962). A very common method to determine the prevailing flow regime in fluidized beds is the stability criterion suggested by Wallis (1962) and Gibilaro (2001). Wallis (1962) and Gibilaro (2001) presented stability as a dimensionless number directly related to fluidized bed porosity and the various fundamental particle and liquid properties (particle and fluid densities, fluid viscosity, and particle diameter). For a detailed explanation of the Wallis stability criterion, the reader is referred to Gibilaro (2001). The criterion for glass beads fluidized with water is as follows:

$$
\begin{aligned}
& \left(\frac{1.79}{n}\right)\left(\frac{\boldsymbol{g} d_{p}}{\boldsymbol{v}_{t}^{2}}\right)^{0.5}\left(\frac{\rho_{p}-\rho_{f}}{\rho_{p}}\right)^{0.5}\left(\frac{\varepsilon_{f}^{1-n}}{\left(1-\varepsilon_{f}\right)^{0.5}}\right)-1 \\
& =\left\{\begin{array}{ccc}
\left\{\begin{array}{c}
>\text { 0: Homogeneous } \\
0: \text { Stability Limit } \\
<0: \text { Bubbling }
\end{array}\right. & \text { for } & d_{p}<0.0012 \mathrm{~m} \\
\text { Indeterminate Stability } & \text { for } & d_{p}>0.0012 \mathrm{~m}
\end{array}\right.
\end{aligned}
$$

where $n$ and $\boldsymbol{v}_{\boldsymbol{t}}$ (terminal velocity) may be calculated as follows:

$$
\begin{gathered}
n=\frac{4.8+2.4 \alpha}{\alpha+1} \\
\alpha=0.043 A r^{0.57} \\
\boldsymbol{v}_{\boldsymbol{t}}=\frac{R e_{t} \mu_{f}}{d_{p} \rho_{f}}
\end{gathered}
$$

where $A r$ is Archimedes number and $R e_{t}$ is the terminal Reynold's number. $R e_{t}$ can be calculated using the Dallavalle (1948) correlation, applicable to creeping and inertial flow regimes:

$$
R e_{t}=\left[-3.809+\left(3.809^{2}+1.832 A r^{0.5}\right)^{0.5}\right]^{2}
$$


As explained by Gibilaro (2001), indeterminate stability may be observed at specific combinations of liquid and solid densities, liquid viscosity, and particle size. For the case of glass beads and water fluidization, Gibilaro (2001) reported that indeterminate stability may be observed for particle diameters roughly greater than $0.0012 \mathrm{~m}$. This is because the stability values are expected to be close to zero for any given porosity. The author explained that in this region, perturbation growth/decay rates are so small that the observed fluidization behavior cannot be easily identified as either stable or unstable. For the case of glass beads and water fluidization, Gibilaro (2001) presented a range of particle diameter sizes where the indeterminate stability region is encountered; this can be seen in Figure 5. As is evident from this figure, the studied case is clearly located within the indeterminate stability region.

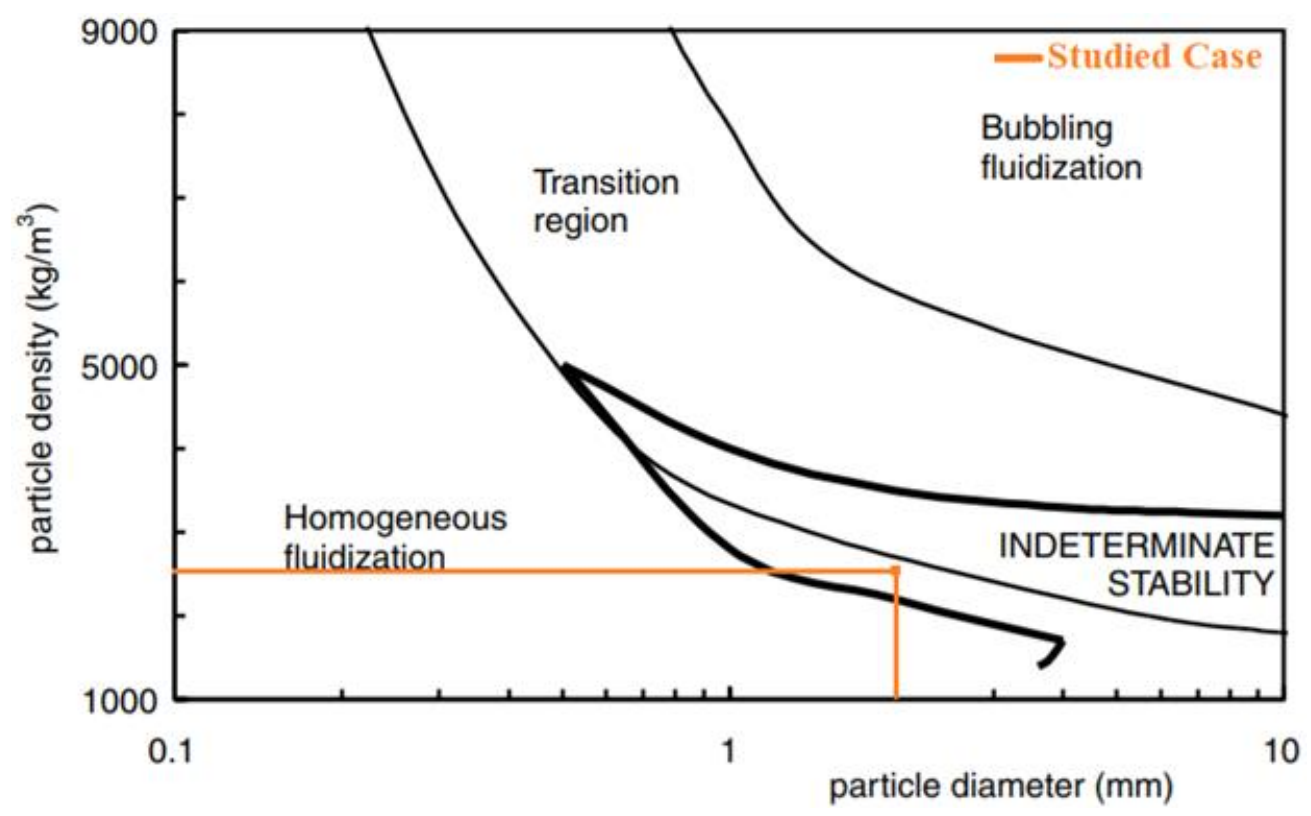

Figure 5: The various achievable flow regimes for particles of different densities and diameters fluidized with water (Gibilaro, 2001).

The next and final section of this chapter presents a detailed literature review of the application of the CFD-DEM simulation method as applied to LSFB processes. The discussion will focus on the gaps that are currently present in the literature. 


\subsection{Application of CFD-DEM in LSFB Literature}

This section presents all relevant studies related to the application of the CFD-DEM method in the simulation of LSFBs. In accordance with the objective of this study the literature review mainly focused on: The ERT experimental method, the influence of various interaction forces and particleparticle contact forces on fluidization behavior, bed expansion behavior and flow regimes, and the effect of liquid superficial velocity on particle and liquid flow behavior.

Many experimental methods have been used to investigate LSFBs, and the reader is referred to section 2.2 for an overview of such experimental techniques. Among the aforementioned techniques, ERT has been commonly used for liquid-solid (two-phase) systems due to its simplicity and its ability to provide visualizations of particle concentrations without disturbing the flow (Kazemzadeh, 2016; Pakzad et al., 2008; Hosseini et al., 2010; Mishra \& Ein-Mozaffari, 2016; Mishra \& Ein-Mozaffari, 2017). Hou et al. (2001) conducted ERT experiments to study mixing characteristics in a magnetically stabilized LSFB. Razzak et al. $(2007,2009)$ successfully employed ERT to determine gas and solids holdup and their radial distribution in a gas-liquidsolid fluidized bed. Furthermore, Razzak et al. (2010) investigated axial hydrodynamics in gasliquid-solid fluidization. Although ERT is highly prevalent in literature concerning multi-phase flows, the application of ERT to study LSFBs remains relatively scarce in literature.

The inclusion of interaction forces in CFD-DEM simulations has been shown to affect the hydrodynamic behavior of LSFBs (Malone et al., 2006). Among the interaction forces between the particles and the fluid, the drag force is considered to be the main interaction force, and is the driving force for fluidization (Deen et al., 2007). Although numerous studies state the significance of selecting an accurate drag model (Beetstra et al., 2007; Lundberg \& Halvorsen, 2008; Van der Hoef et al., 2008), the influence of different drag models on LSFB performance have yet to be analyzed. A summary of the drag models employed in CFD-DEM simulations of LSFB found in literature is presented in Table 1. Malone et al. (2006) showed that LSFB simulations with only drag force resembled gas-solid fluidization, and the addition of other interaction forces including the pressure gradient, virtual mass, and Magnus lift forces changed the bed behavior to visually resemble liquid-solid fluidization. However, their findings were not supported experimentally. In a few studies, interaction forces (other than drag) were neglected without any reasoning (AlArkawazi et al., 2017; Sen et al., 2014). Peng et al., $(2014,2016)$ neglected interaction forces other 
than drag and pressure gradient and stated that their formulations were similar to Anderson \& Jackson (1967) for initial derivation of the governing equations; the influence of other interaction forces on simulation results were not addressed. Di Renzo \& Di Maio (2007) and Di Renzo et al. (2011) neglected interaction forces other than drag and pressure gradient, and rationalized that their motivation was due to the lack of reliability and accuracy of formulations. Nonetheless, the importance of virtual mass force in LSFB processes was discussed in great detail by Ghatage et al. (2014), and was shown to drastically affect LSFB stability, especially in the transition from homogeneous to heterogeneous flow regimes. Malone et al. (2006, 2007, 2008) included the Magnus lift force in their CFD-DEM simulations of a LSFB, however, Blais \& Bertrand (2015) comprehensively argued that Magnus force can be neglected in liquid-solid systems as particle rotation is hindered to a great degree by the surrounding liquid. No study has been conducted to date in CFD-DEM simulations of LSFBs that included Saffman lift force. In addition, a consensus can be found in literature regarding the validity of the omission of Basset force in CFD-DEM simulations of LSFBs. The explanation was due to the lack of reliable formulations (Wang et al., 2012), and the increase in computational time (Moreno-Casas \& Bombardelli, 2016). A summary of the inclusion and omission of interaction forces in CFD-DEM simulations of LSFB literature is presented in Table 1. As summarized in Table 1, the interaction forces are usually selected without any profound explanation, and the contribution of individual interaction force to simulation accuracy have yet to be analyzed. 
Table 1: Interaction forces as included in literature of CFD-DEM simulations of LSFB

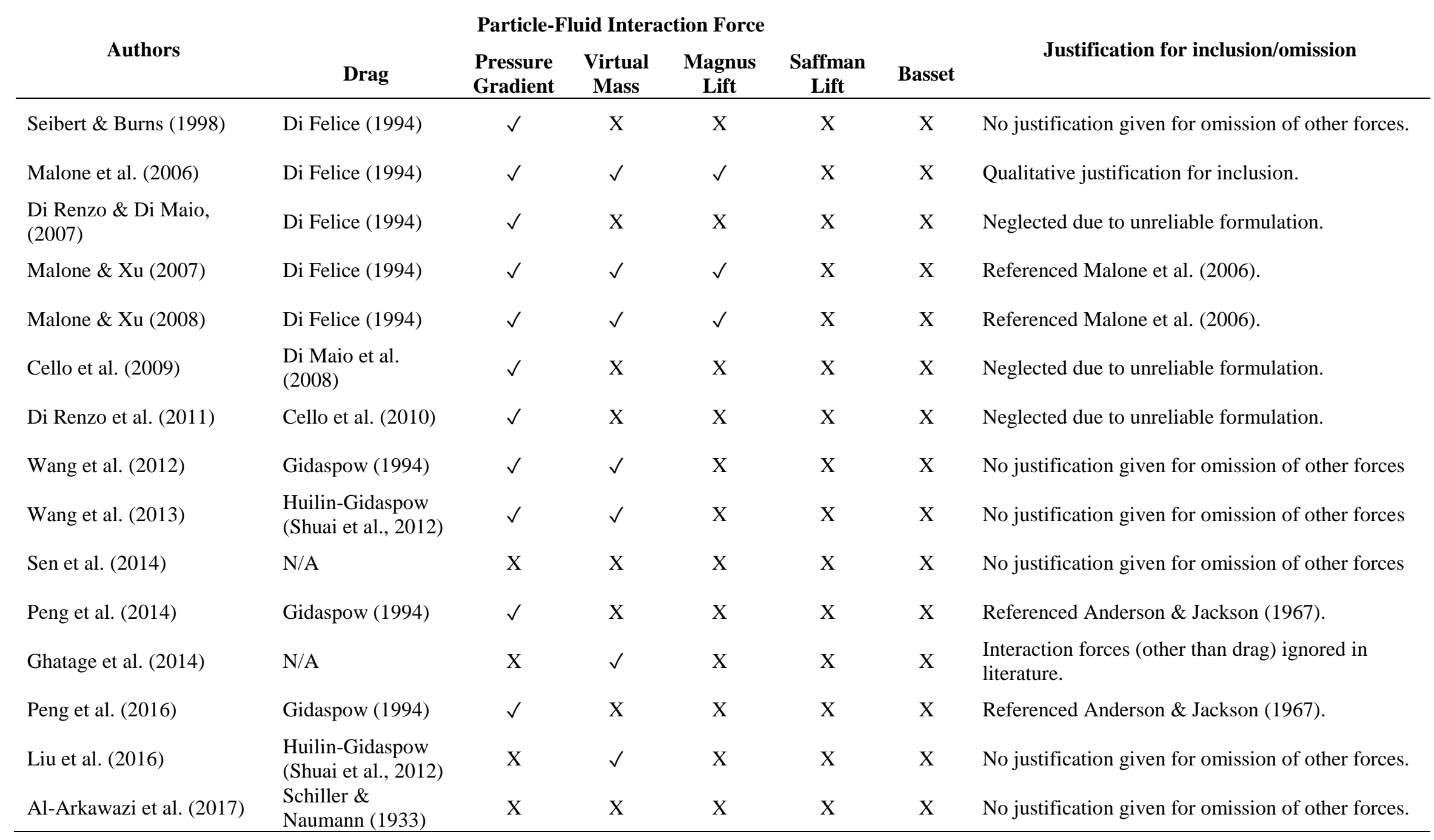


In solid-liquid systems, the presence of an interstitial liquid between particles and particlegeometry has been shown to drastically change particle-particle and particle-geometry contact characteristics compared to gas-solid systems (Ma et al., 2015). It has been discussed that the kinetic energy of particles immersed in liquid can be dissipated by viscous stress imposed on them by the liquid in addition to contact inelasticity (Li et al., 2012). Moreover, the liquid surrounding the particles can change the particles' rotational characteristics as well as the friction between them (Buck et al., 2017). However, the use of dry particle contact parameters (i.e. the coefficient of restitution $(\mathrm{COR})$, coefficient of sliding friction $\left(\mu_{\mathrm{s}}\right)$, and coefficient of rolling friction $\left(\mu_{\mathrm{r}}\right)$ is highly prevalent in CFD-DEM simulations of LSFBs (Al-Arkawazi et al., 2017; Di Renzo et al., 2011; Di Renzo \& Di Maio, 2007; Ghatage et al., 2014; Peng et al., 2014, 2016). Wang et al., (2012) and Sen et al. (2014) reduced the COR to one constant value of 0.3, and 0.2, respectively, to account for the presence of surrounding liquid, however, no further calibration was conducted. Gollwitzer et al. (2012) and Joseph et al. (2001) developed an equation for COR based on stokes number, to account for the viscous dissipation in solid-liquid systems. However, the Liu et al. (2016) study of a 2D LSFB showed that the discrepancy between the simulation and experimental data decreased minimally when the "dynamic" COR introduced by Gollwitzer et al. (2012) was used compared to the cases when dry particles COR was utilized. Blais \& Bertrand (2017) calibrated contact parameters individually and observed that varying $\mu_{\mathrm{s}}$ and $\mu_{\mathrm{r}}$ played a key role in particle suspension in viscous solid-liquid mixing vessels, noting that varying the COR had minimal effects on particle suspension. Though the authors only tested the two extreme values of COR (i.e. 0.9 and 0.01), and more intermediate COR values were not investigated. Di Renzo \& Di Maio (2007) and Di Renzo et al. (2011) explained that in liquid-solid fluidization, the simulation time can be significantly reduced by employing a less than realistic Young's Modulus. The authors applied a Young's Modulus value of $10^{7} \mathrm{~Pa}$ in their CFD-DEM simulations, and reported that this did not influence their simulation results. A systematic calibration of particle contact parameters in liquid-solid fluidization has yet to be conducted in order to study the influence of each parameter on simulation accuracy.

The hydrodynamics of a LSFB has been shown to be a strong function of the flow regime. A common method to determine the flow regime in fluidized beds is the Wallis (1962) stability criterion. The Wallis criterion is directly related to fluidized bed porosity, and the bed porosity itself is directly influenced by the fluid superficial velocity and the number of particles. Di Renzo 
\& Di Maio (2007) conducted CFD-DEM simulations of a 2D gas-solid fluidized bed. Using the Wallis (1962) stability criterion, the authors successfully identified the particle volume fraction (PVF) at which the transition from homogeneous to bubbling regime occurred. The authors also conducted CFD-DEM simulations of a 2D LSFB and reported a constant spatially averaged PVF value along the bed height for all simulation conditions studied, which is the characteristic of homogeneous fluidization. Di Renzo et al. (2011) observed the homogeneous flow regime for all cases studied, and showed that the locally moving vortices are the main cause for the homogeneous distribution of particles throughout the bed in CFD-DEM simulation of a 2D LSFB. Similar to Di Renzo \& Di Maio (2007) study, Wang et al. (2012) also reported a constant PVF value along the bed height in their simulations. Their findings implied the homogeneous flow regime for all cases studied, however, bubbling behavior could be easily identified by observing their instantaneous snapshots; this was not addressed by the authors. Liu et al. (2016) conducted 2D simulations of a LSFB for water and $0.002 \mathrm{~m}$ glass beads where intermediate behavior is expected based on Wallis (1962) criterion regardless of the liquid superficial velocity. The authors reported a constant distribution of particles along the bed at various liquid superficial velocities for $0.002 \mathrm{~m}$ particles implying the presence of the homogeneous flow regime. Their conclusion of homogeneous flow regime may be doubtful due to the $2 \mathrm{D}$ nature of the simulations. Ghatage et al. (2014) employed a different stability criterion, termed the 1D linear stability criterion suggested by Joshi et al. (2001), and showed that this criterion could identify the point of transition from homogeneous to bubbling regimes in a 3D LSFB. Furthermore, the authors showed a highly random and irregular trend in the radial PVF in the unstable (bubbling) flow regime. Although some studies have investigated the hydrodynamic behavior in homogeneous and bubbling flow regimes, the hydrodynamic behavior of LSFB systems operating in the intermediate flow regime remains lacking in literature.

The influence of liquid superficial velocity on the hydrodynamic behavior of a LSFB has also been investigated in some studies. Di Renzo et al. (2011) and Peng et al. (2016) conducted CFD-DEM simulations on a bi-dispersed LSFB and found that greater particle segregation is attainable with an increase in liquid superficial velocity. Wang et al. (2012) found that the liquid and particle axial velocities differ in magnitude and trend with varying liquid superficial velocities. Liu et al. (2016) also reported varying trends in axial particle velocity profiles with a change in liquid superficial velocity when studying a 2D LSFB. Although the weight fraction of particles (i.e. number of particles) has been shown to significantly influence the flow behavior in multiphase systems such 
as gas-liquid-solid fluidized beds (Fan et al., 1985) and gas-solid downers (Zhao et al., 2010), a detailed investigation has yet to be performed to study the influence of particle weight fraction on the hydrodynamics of a LSFB.

A summary of important parameters used in literature of CFD-DEM simulations of LSFBs are presented in Table 2 below for comparison. 
Table 2: CFD-DEM studies on liquid-solid fluidized beds published to date

\begin{tabular}{|c|c|c|c|c|c|c|c|c|c|c|c|c|c|c|c|c|}
\hline \multirow{2}{*}{ Authors } & \multicolumn{2}{|c|}{$\begin{array}{c}\text { Column } \\
\text { Specifications }\end{array}$} & \multicolumn{2}{|c|}{ Liquid phase } & \multicolumn{4}{|c|}{ Solid Phase } & \multicolumn{2}{|c|}{$\begin{array}{c}\text { Number of } \\
\text { particles }\end{array}$} & \multicolumn{2}{|c|}{$\begin{array}{c}\text { Friction } \\
\text { Coefficient }\end{array}$} & \multirow{2}{*}{$\begin{array}{c}\text { Spring } \\
\text { Stiffness } \\
\\
\kappa_{n}(N / m)\end{array}$} & \multicolumn{2}{|c|}{$\begin{array}{l}\text { Restitution } \\
\text { coefficient }\end{array}$} & \multirow{2}{*}{$\begin{array}{c}\text { Time Step } \\
\text { t (s) }\end{array}$} \\
\hline & $\begin{array}{c}\mathrm{D} \\
(\mathbf{c m})\end{array}$ & $\begin{array}{c}\mathbf{H} \\
(\mathbf{c m})\end{array}$ & $\begin{array}{c}\rho f \\
(\mathrm{~kg} / \mathrm{m3})\end{array}$ & $\begin{array}{c}\mu\left(\mathbf{x} 10^{-3}\right. \\
\text { Pa.s) }\end{array}$ & $d_{p 1}(\mathbf{m m})$ & $\begin{array}{c}\mathbf{d}_{\mathrm{p} 2} \\
(\mathbf{m m})\end{array}$ & $\rho_{p 1}(\mathrm{~kg} / \mathrm{m3})$ & $\begin{array}{c}\rho_{\mathrm{p} 2} \\
\left(\mathrm{~kg} / \mathrm{m}^{3}\right)\end{array}$ & $\mathbf{N}_{p 1}$ & $\mathrm{~N}_{\mathrm{p} 2}$ & $\mu_{\mathrm{s}}$ & $\mu_{\mathrm{r}}$ & & $\begin{array}{l}\text { COR } \\
(\mathbf{P}-\mathbf{P})\end{array}$ & $\begin{array}{l}\text { COR } \\
(\mathbf{P}-W)\end{array}$ & \\
\hline $\begin{array}{l}\text { Siebert \& Burns } \\
\text { (1998) }\end{array}$ & 1.5 & 20 & 1000 & 1 & 0.15 & 0.2 & $2000-2900$ & 2450 & 500 & 500 & NA & NA & NA & NA & NA & NA \\
\hline $\begin{array}{l}\text { Malone et al. } \\
\text { (2006) }\end{array}$ & 20 & 100 & 1000 & 1 & 5 & none & 2750 & none & 1600 & none & 0.3 & 0.3 & $1.5 \times 10^{6}$ & NA & NA & $5 \times 10^{-7}$ \\
\hline $\begin{array}{l}\text { Di-Renzo \& Di- } \\
\text { Maio (2007) }\end{array}$ & 1.8 & 8 & 1000 & 1 & 0.2 & none & 2500 & none & 15000 & none & 0.3 & 0.3 & NA & 0.9 & 0.9 & $1 \times 10^{-5}$ \\
\hline $\begin{array}{l}\text { Malone et al. } \\
\text { (2007) }\end{array}$ & 15 & 130 & 1000 & 1 & 5 & 2.5 & 2750 & 3200 & 1000 & 8000 & 0.3 & 0.3 & $4 \times 10^{4}$ & wet & wet & $5 \times 10^{-7}$ \\
\hline $\begin{array}{c}\text { Malone \& Xu } \\
\text { (2008) }\end{array}$ & 5 & 70 & 1000 & 1 & 1.5 & none & 11000 & none & 2000 & none & 0.3 & 0.3 & $4.7 \times 10^{4}$ & NA & NA & $1 \times 10^{-6}$ \\
\hline $\begin{array}{c}\text { Cello \& Di-Renzo } \\
\text { (2009) }\end{array}$ & 5 & 70 & 1000 & 1 & 0.214 & 0.776 & 2450 & 1500 & $\mathrm{Na}$ & $\mathrm{Na}$ & 0.3 & 0.3 & NA & NA & NA & NA \\
\hline $\begin{array}{l}\text { Di-Renzo et al. } \\
\text { (2011) }\end{array}$ & 4 & 50 & $\begin{array}{l}1000 \\
1070\end{array}$ & $1,0.14$ & 0.65 & 1.08 & 3950 & 2910 & 9938 & 2064 & 0.3 & 0.3 & $\begin{array}{l}0.1 \mathrm{GPa} \\
\text { (Young's } \\
\text { Modulus) }\end{array}$ & 0.9 & 0.9 & $5 \times 10^{-6}$ \\
\hline Wang (2012) & 14 & 50 & 998.2 & 1 & 3 & none & 2500 & none & 9500 & none & 0.3 & 0.3 & 800 & NA & NA & $1 \times 10^{-4}$ \\
\hline Wang (2013) & 10 & 150 & 998.2 & 1 & 2 & 2 & 2540 & 2540 & 1400 & 700 & NA & NA & 2000 & 0.3 & 0.3 & $1 \times 10^{-4}$ \\
\hline Sen et al. (2014) & 10 & 29 & 1000 & 1 & 2 & none & 1030 & none & 50000 & none & 0.5 & 0.01 & NA & 0.2 & 0.2 & $3.3 \times 10^{-5}$ \\
\hline Peng et al. (2014) & 5 & 100 & 998.2 & 1 & 3,5 & $6,5,4$ & 2300 & 7800 & 17000 & 1 & 0.3 & 0.018 & 10000 & 0.9 & 0.9 & $5 \times 10^{-5}$ \\
\hline $\begin{array}{c}\text { Ghatage et al. } \\
\text { (2014) }\end{array}$ & 5 & 150 & 998.2 & 1 & 5 & $6,7,8,9$ & 2300 & 7800 & 3600 & 1 & 0.3 & 0.018 & 10000 & 0.9 & 0.9 & $5 \times 10^{-5}$ \\
\hline Peng et al. (2016) & 2 & 40 & 998.2 & 1 & 1.09 & 1.09 & 1600 & 1900 & NA & NA & 0.35 & 0.075 & 10000 & 0.9 & 0.9 & $1 \times 10^{-5}$ \\
\hline Liu et al. (2016) & 5.12 & 204 & 998.2 & 1 & $\begin{array}{c}2,3,4,6 \\
\text { (glass), } \\
4.5 \\
\text { (steel) }\end{array}$ & none & $\begin{array}{c}2540 \text { (glass), } \\
7780 \text { (steel) }\end{array}$ & none & $\begin{array}{l}5000 \\
\text { or less }\end{array}$ & none & NA & NA & $\begin{array}{l}50.5 \mathrm{GPa} \\
\text { (Young's } \\
\text { Modulus) }\end{array}$ & $0.9 /$ wet & $0.9 /$ wet & $10^{-3} / 10^{-4}$ \\
\hline $\begin{array}{l}\text { Al-Arkawazi et al. } \\
\qquad(2017)\end{array}$ & $\begin{array}{l}9.6 \\
4.2\end{array}$ & $\begin{array}{l}100 \\
100\end{array}$ & 998.2 & 1 & 2 & none & 2300 & none & 2400 & none & 0.3 & 0.3 & $\begin{array}{l}70 \mathrm{GPa} \\
\text { (Young's } \\
\text { modulus) }\end{array}$ & 0.9 & 0.9 & $1 \times 10^{-4}$ \\
\hline
\end{tabular}




\subsection{Research Objectives}

From the literature review presented above, it was apparent that research gaps exist in analyzing LSFB systems. This study will attempt to address these gaps, and the objectives of this research study are as follows:

- Conduct ERT experiments to study the LSFB system, and compare the experimental and simulation results. The application of ERT experiments to study LSFBs remains lacking in literature.

- Investigate various drag models, as the drag model is often simply selected in literature. The influence of applying different drag models on LSFB performance is lacking in literature.

- Perform interaction force analysis as the individual interaction forces (i.e. pressure gradient, virtual mass, and Saffman lift forces) are usually selected without any profound explanation. Their individual contribution to simulation accuracy have yet to be analyzed.

- Explore the influence of individual contact parameter on CFD-DEM simulation accuracy (i.e. coefficient of restitution, coefficient of rolling friction, and the coefficient of sliding friction). Such parameters employed in available literature often do not account for the particles being wet. A systematic calibration of particle contact parameters in liquid-solid fluidization has yet to be conducted in order to study the influence of each parameter on simulation results.

- Assess the hydrodynamic behavior of LSFB systems operating within the intermediate flow regime, as such information remains lacking in literature.

- Investigate the influence of the liquid superficial velocity and the number of particles on the hydrodynamic behavior of a 3D LSFB, as such analysis have yet to be determined.

The next chapter presents the research methodology which was designed to address the above gaps in literature. 


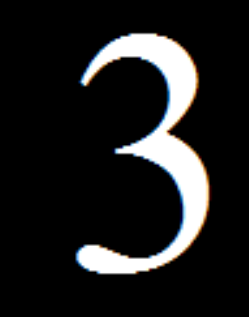

\section{Experimental}

\section{and}

Simulation

Methodologies 


\subsection{Introduction}

This chapter presents the experimental and simulation methodologies applied to study the hydrodynamic behavior in a LSFB. The ERT experimental method was explained in section 2.2.5, and the reader is referred to that section for an in-depth explanation regarding ERT components and methodology. Furthermore, this chapter presents the CFD-DEM simulation setup including the mesh used and the initial contact parameters employed.

\subsection{ERT Experimental Setup}

The schematic diagram of the LSFB used in this study can be seen in Figure 6 and Figure 7. By utilizing a centrifugal pump, water was pumped from its holding tank through a set of two rotameters. A recycle globe valve was used to control the liquid velocity. A calming section was installed below the fluidization column, and packed with $0.003 \mathrm{~m}$ particles to ensure the generation of a uniform water velocity profile in the LSFB. Directly above the calming section, a distributor was placed to further control water distribution, and to prevent the fluidizing particles from falling through to the calming section. The fluidization column was $0.8 \mathrm{~m}$ in height and $0.1 \mathrm{~m}$ in diameter. The solid particles used in this study were mono-dispersed $0.002 \mathrm{~m}$ glass beads with $2500 \mathrm{~kg} / \mathrm{m}^{3}$ density. The particle size was chosen to be $0.002 \mathrm{~m}$ since it was desired to produce the intermediate flow regime, as previously discussed in section 2.5. The variables used in the experiments were: fluid superficial velocity (factor A) and number of particles (factor B). The experimental variables and their levels are summarized in Table 3.

Table 3: Experimental design

\begin{tabular}{ccc}
\hline Particle Size & Liquid velocity $(\mathrm{A})(\mathrm{m} / \mathrm{s})$ & Number of Particles $(\mathrm{B})$ \\
\hline & 0.12 & \\
& 0.16 & 50,000 \\
0.20 & \\
$0.002 \mathrm{~m}$ & 0.12 & 75,000 \\
& 0.16 & \\
& 0.20 & 100,000 \\
& 0.12 & \\
\hline
\end{tabular}

Connected to the fluidization column were four tomography sensor planes. Each plane consisted of 16 equally spaced electrodes and the planes were placed $0.06 \mathrm{~m}$ apart. The planes were numbered one through four (P1 to P4) from top to bottom, and the bottom-most plane was placed 
$0.06 \mathrm{~m}$ above the distributor. Each sensor plane was connected to the data acquisition system (DAS), and the latter was connected to a computer for data storage and image reconstruction. 


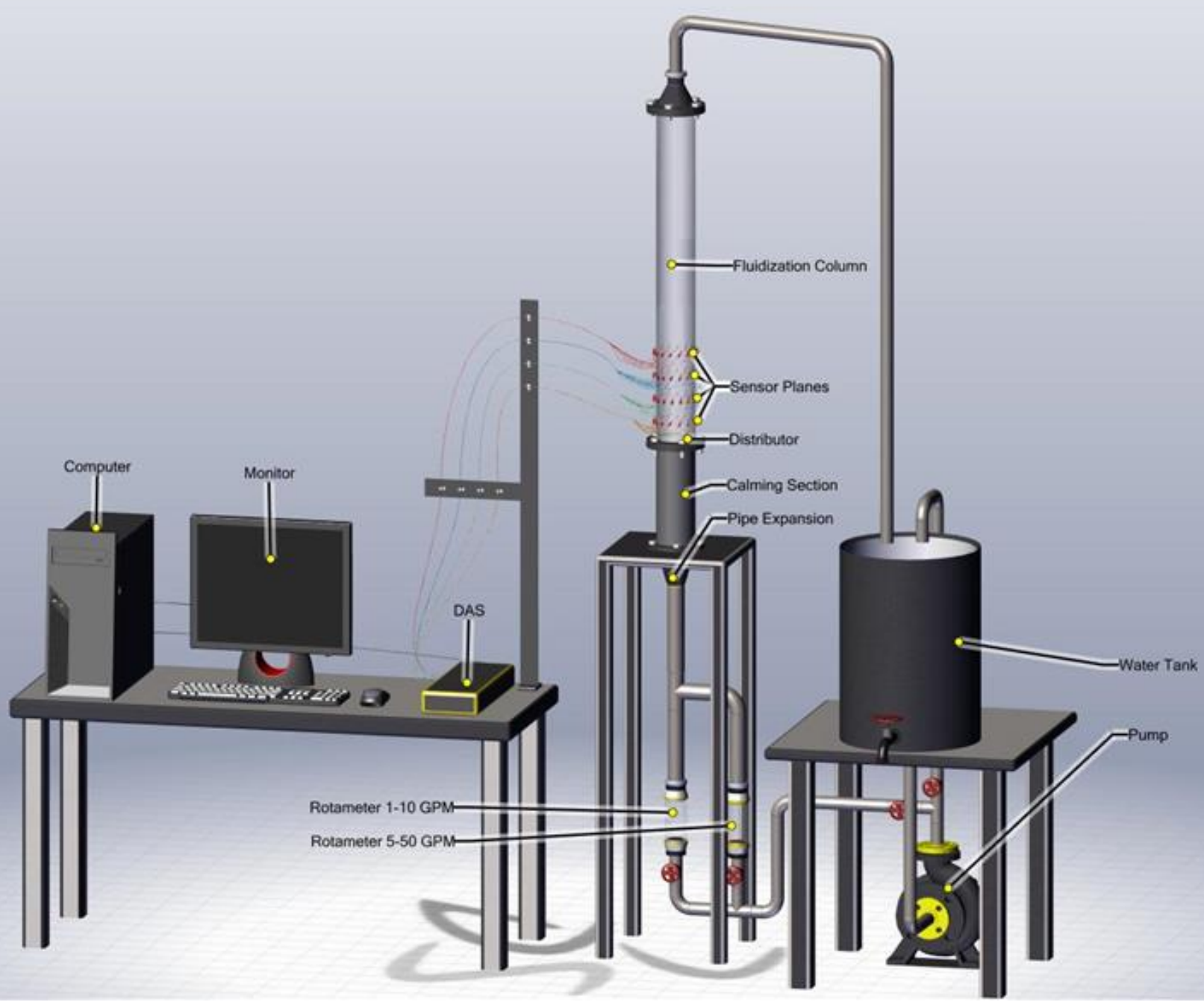

Figure 6: Experimental Setup.

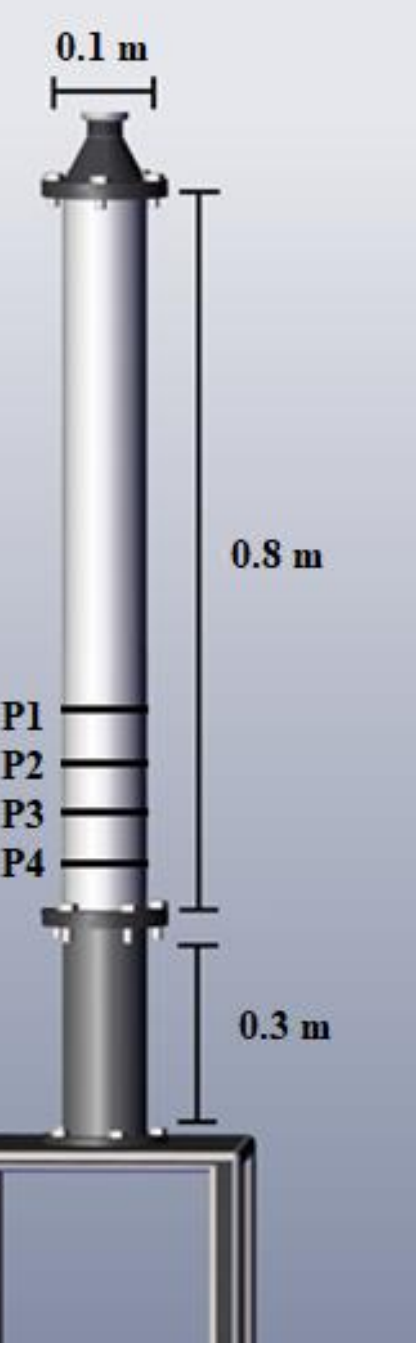

Figure 7: Fluidized bed dimensions. 
The ERT experimental settings used in this study are presented in Table 4. ERT results were provided as a set of contour plots, as well as spatially averaged planar particle volume fractions (PPVF). The reproducibility of experiments was checked by repeating each experimental run three times. A set of ERT results is presented in Figure 8. The temporally averaged PPVF (\%) values at each plane were used for quantitative comparison between experimental and simulation results.

Table 4: ERT Experimental Settings

\section{DAS Settings}

\section{Data collection}

\begin{tabular}{cccc}
\hline Number of Sensing Planes & 4 & Frames per reference & 50 \\
Electrodes per plane & 16 & Sampling time interval (ms) & 50 \\
Samples per frame & 8 & Maximum number of frames & 50 \\
Injection current (mA) & 1.5 & & \\
\hline
\end{tabular}

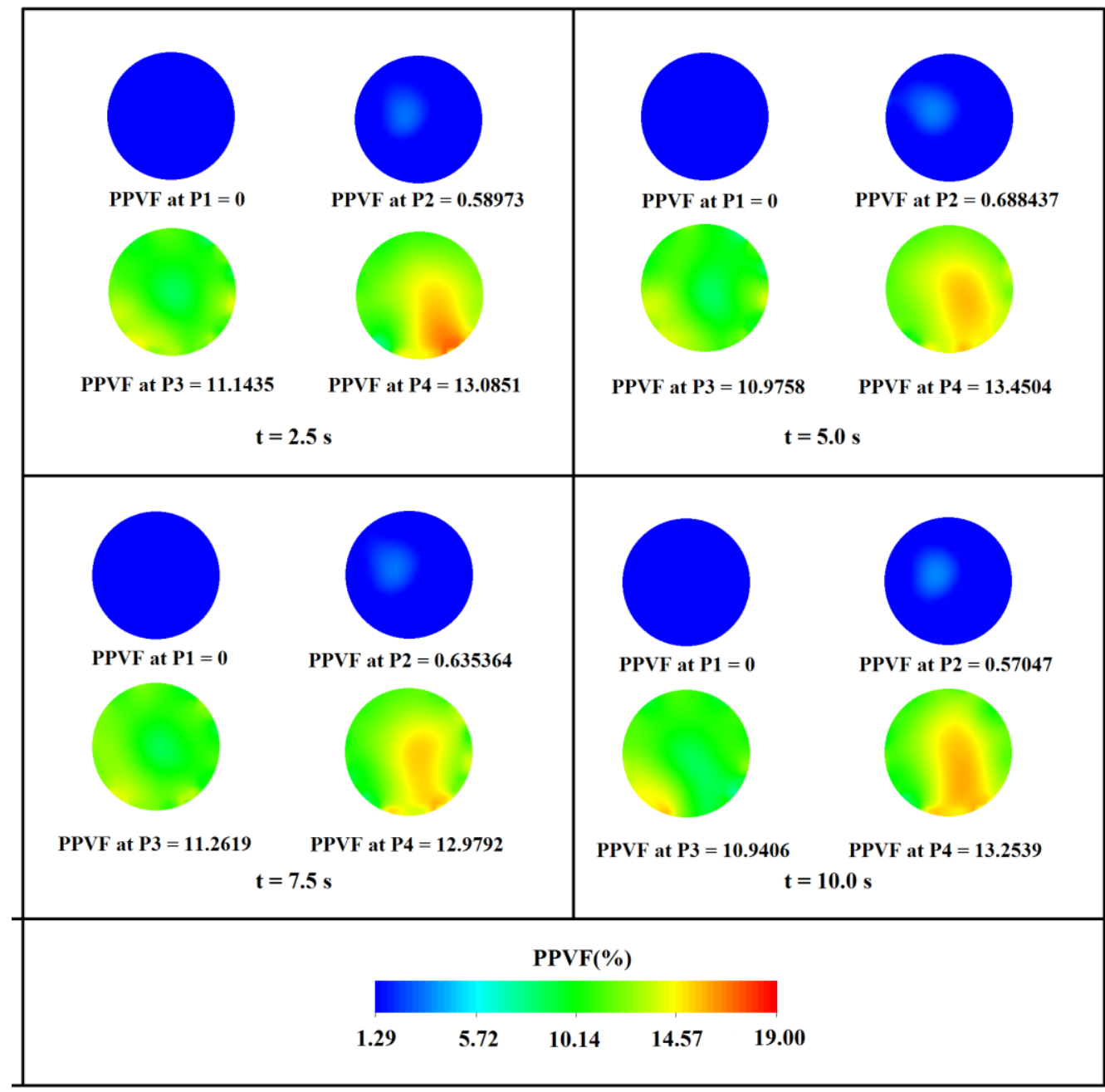

Figure 8: Instantaneous ERT results for 50,000, $0.002 \mathrm{~m}$ particles, fluidized at $0.20 \mathrm{~m} / \mathrm{s}$ at $2.5 \mathrm{~s}, 5.0 \mathrm{~s}, 7.5$ $\mathrm{s}$, and $10.0 \mathrm{~s}$ timed pursuant to visual confirmation of steady state conditions. 


\subsection{CFD-DEM Simulation Setup}

The simulation geometry was created to replicate that of the experiments; $0.8 \mathrm{~m}$ in height, and 0.1 $\mathrm{m}$ in diameter. A tetrahedral unstructured mesh with 21040 tetrahedral elements and 12312 nodes was selected for the simulations (Figure 9). This mesh satisfied the mesh criterion of the CFDDEM approach in which the mesh element volume must be larger than particle volume (Anderson \& Jackson, 1967). The fluid velocity profiles obtained from this mesh were compared with those obtained from other finer meshes with 36800, 84640, and 350640 tetrahedral elements, and minimal differences between fluid velocity profiles were observed.

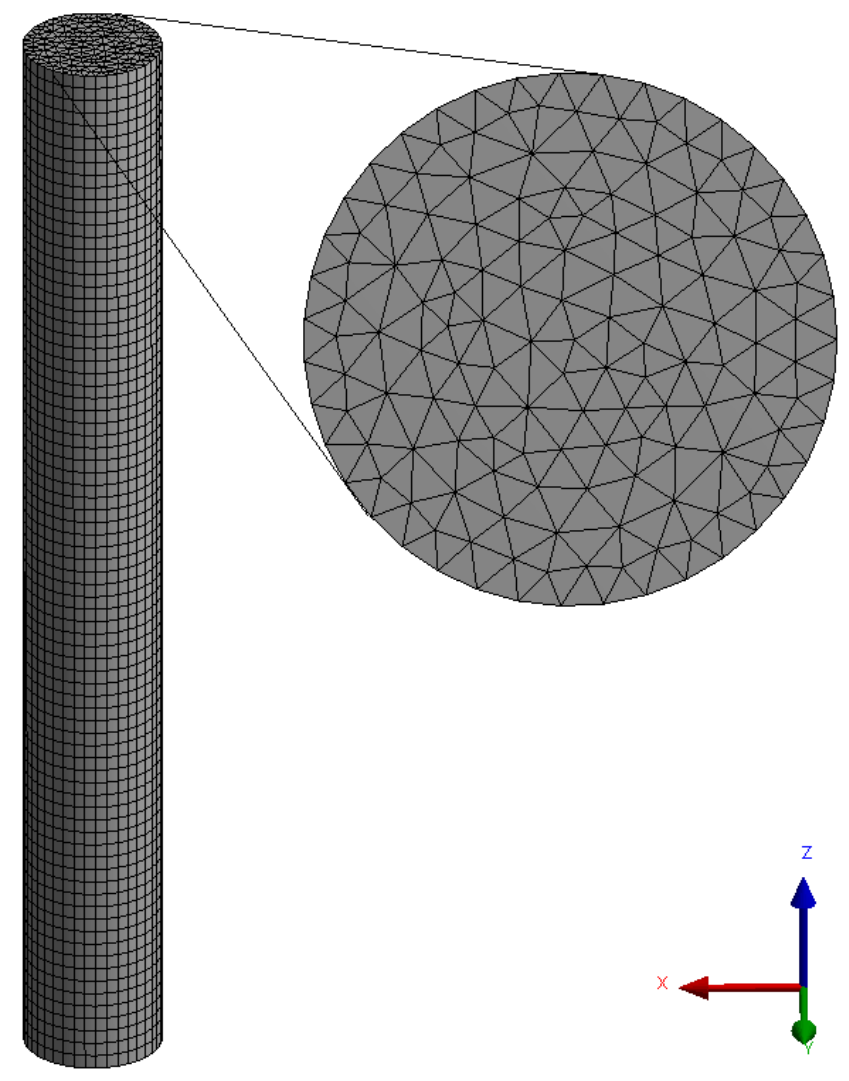

Figure 9: Computational geometry and mesh.

In the current study, the drag force analysis, interaction force analysis, and particle contact parameter calibration were performed with 50,000, $0.002 \mathrm{~m}$ particles, fluidized at $0.16 \mathrm{~m} / \mathrm{s}$. Once such analysis was done, the liquid superficial velocity and the number of particles were varied to investigate the influence of such parameters on the hydrodynamic behavior within the LSFB. In all simulations, the particle diameter was maintained constant $(0.002 \mathrm{~m})$, the DEM time-step was 
set to $23 \%$ Rayleigh time, which was equivalent to $2.00 \mathrm{E}-5 \mathrm{~s}$, and the CFD time-step was 100 times larger than the DEM time-step. Table 5 summarizes all simulation parameters applied for FLUENT and EDEM.

Table 5: Particle, geometry, and Fluid properties

\section{CFD-DEM Simulations parameters}

\section{Geometry}

Material

Density $\left(\mathrm{kg} / \mathrm{m}^{3}\right)$

Height (m)

Width (m)

\section{Fluent (Eulerian phase)}

Material

Density $\left(\mathrm{kg} / \mathrm{m}^{3}\right)$

Viscosity (Pa.s)

Velocity (m/s)

Turbulence Model
PVC

1400

0.8

0.01

EDEM (Lagrangian phase)

Material

Density $\left(\mathrm{kg} / \mathrm{m}^{3}\right)$

Particle diameter (m)

Number of particles

Water
1000
1.002 E-3
0.16
$k-\varepsilon$ model

Glass

2500

0.002

50,000

As the particle material used in Blais \& Bertrand (2017) study was identical to those used in this study, their initial particle-particle (P-P) choice of contact parameters was applied. However, in this study, the experimental apparatus material was different than that of the particles, which was not the case for Blais \& Bertrand (2017). As a result, it was observed that employing a 0.9 COR (P-W) (as suggested by Blais \& Bertrand (2017)) caused less stable simulations. Therefore, the initial COR $(\mathrm{P}-\mathrm{W})$ value was reduced with the hindsight that COR $(\mathrm{P}-\mathrm{W})$ and all other particle contact parameters will be calibrated in later simulations. The value of $0.9 \mathrm{COR}(\mathrm{P}-\mathrm{W})$ was nonetheless investigated to quantify its effect on simulation results. The Young's Modulus value of $10^{7} \mathrm{~Pa}$ employed in this study was based on Di Renzo et al. (2011). The P-P and P-W contact parameter values initially used in the simulations are presented in Table 6. 
Table 6: Initial contact parameters

\section{Contact Parameters}

\begin{tabular}{ll}
\hline Coefficient of Restitution & $0.25(\mathrm{P}-\mathrm{W}), 0.9(\mathrm{P}-\mathrm{P})$ \\
Coefficient of sliding friction & $0.3(\mathrm{P}-\mathrm{W}), 0.3(\mathrm{P}-\mathrm{P})$ \\
Coefficient of rolling friction & $0.1(\mathrm{P}-\mathrm{W}), 0.1(\mathrm{P}-\mathrm{P})$ \\
Young's Modulus $(\mathrm{Pa})$ & $10^{7}$ \\
Poison's ratio & $0.4(\mathrm{P}-\mathrm{W}), 0.25(\mathrm{P}-\mathrm{P})$ \\
\hline
\end{tabular}

Each simulation was run for 20 s of simulation time. The fluidization behavior was observed to have reached steady state after $10 \mathrm{~s}$ of simulation time; steady state conditions were confirmed visually. Time averaged PPVF values at steady state conditions at $0.06 \mathrm{~m}$ and $0.12 \mathrm{~m}$ were obtained as the simulation output (using a MATLAB code, see Appendix Figure 31), and compared to PPVF values retrieved from ERT experiments at identical conditions. The steady state condition for ERT experiments was confirmed by observing the PPVF values over time and noting no significant changes $( \pm 0.5 \mathrm{PPVF}(\%)$ standard deviation). 


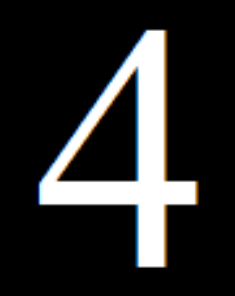

Results and

Discussion 


\subsection{Introduction}

Based on the experimental and simulation setups discussed in chapter three, this chapter sequentially addresses: the design of experiments (section 4.1, includes statistical analysis and experimental results), the effect of various interaction forces (drag force (section 4.2), other interaction forces (section 4.3) on simulation results, and the systematic calibration of contact parameters (section 4.4). These sections were followed by an in-depth study which aims to compare the local particle volume fraction values obtained experimentally and through simulations (section 4.5). Sections 4.6 and 4.7 address the influence of the liquid superficial velocity and the number of particles, respectively, on the hydrodynamic behavior of LSFBs. Sections 4.6 and 4.7 also include LSFB stability analysis which was employed to determine the flow regime at various operating conditions (combinations of liquid superficial velocities and number of particles).

\subsection{Design of Experiments}

Table 7 describes the bed height relative to the sensor planes, as observed during the experimental runs. As expected, the bed height increased with increasing number of particles and increasing fluid flowrate.

Table 7: Experimental observations

\begin{tabular}{ccccc}
\hline $\begin{array}{c}\text { Particle } \\
\text { size }(\mathbf{m})\end{array}$ & $\begin{array}{c}\text { No. of } \\
\text { particles }\end{array}$ & Fluid velocity $(\mathbf{m} / \mathbf{s})$ & Bed height $(\mathbf{m})$ & Comments \\
\hline & \multirow{2}{*}{50,000} & 0.12 & 0.105 & Few particles reach P3 \\
& & 0.16 & 0.185 & Barely reaches P2 \\
& & 0.20 & 0.240 & Barely reaches P1 \\
0.002 & 0.12 & 0.150 & Few particles reach P2 \\
& \multirow{2}{*}{75,000} & 0.16 & 0.235 & Barely reaches P1 \\
& & 0.20 & 0.340 & Reaches past all planes \\
& \multirow{2}{*}{100,000} & 0.12 & 0.240 & Reaches P1 \\
& & 0.16 & 0.310 & Reaches past all planes \\
& 0.20 & 0.450 & Reaches past all planes \\
\hline
\end{tabular}

As previously mentioned, the recorded experimental result was PPVF. However, as seen in Table 7, not all sensor planes were surpassed by the particles for all experimental runs. Therefore, to conduct meaningful analysis, an average of relevant PPVFs at their respective planes was taken, and the criteria for selection of relevant planes was that particles must distinctly clear that plane. For example, Figure 8 shows results for 50,000 particles fluidized at $0.16 \mathrm{~m} / \mathrm{s}$, and Table 7 shows that at these conditions, only P2 sensor plane was reached. Since P4 and P3 sensor planes were 
distinctly cleared, the PPVF values of these two planes were averaged, while the PPVF values at P2 and P1 were ignored. This averaged value represents the bed-averaged particle volume fraction (henceforth, BPVF). The BPVF values for all experimental runs were utilized to develop a statistical model, and are presented in Table 8.

Table 8: BPVF values selected for statistical analysis

\begin{tabular}{cccc}
\hline $\begin{array}{c}\text { Particle } \\
\text { Size }\end{array}$ & $\begin{array}{c}\text { Number of } \\
\text { Particles }\end{array}$ & $\begin{array}{c}\text { Fluid } \\
\text { velocity } \\
(\mathrm{m} / \mathrm{s})\end{array}$ & BPVF (\%) \\
\hline \multirow{4}{*}{50,000} & 0.12 & 22.69 \\
& & 0.16 & 12.34 \\
& & 0.20 & 9.98 \\
& \multirow{2}{*}{75,000} & 0.12 & 23.29 \\
& & 0.16 & 13.66 \\
& \multirow{2}{*}{100,000} & 0.20 & 12.10 \\
& & 0.12 & 30.29 \\
& & 0.16 & 22.77 \\
\end{tabular}

In this study, the RSM D-Optimal design was selected to analyze the effect of fluid superficial velocity (factor A), the number of particles (factor B), and their interaction, on the BPVF in a LSFB. The analysis of variance can be seen in Table 9. Since the p-value for the model was less than 0.05 , the model was significant. The chance of an F-value of 272.86 could occur due to noise was $0.03 \%$. The significant factors were $\mathrm{A}, \mathrm{B}, \mathrm{A}^{2}$ and $\mathrm{B}^{2}$, which implied that the fitted model was quadratic. The RSM D-Optimal design equation was obtained as:

$$
\mathrm{BPVF}=14.11+4.61 \mathrm{~A}-5.96 \mathrm{~B}+3.90 \mathrm{~A}^{2}+3.36 \mathrm{~B}^{2}
$$

Since the interaction between the factors was insignificant, as seen in Table 9, the AB term was removed from the design equation. Eq. 4-1 is a coded design equation, and by default, the levels of each factor were designated $-1,0$, and 1 , for the low, mid, and high levels, respectively. The coefficient of determination, $\mathrm{R}^{2}$, value obtained was 0.9978 , which implies that only $0.22 \%$ of the BPVF response cannot be explained by the model, signifying near identical fit. The model was plotted against experimental results and presented in Figure 10. 
Table 9: ANOVA table

\begin{tabular}{ccccccc}
\hline \multicolumn{7}{c}{ Analysis of Variance Table } \\
\hline Source & Sum of Squares & df & $\begin{array}{c}\text { Mean } \\
\text { Square }\end{array}$ & F Value & $\begin{array}{c}\text { p-value } \\
\text { Prob }> \\
\text { F }\end{array}$ & Significance \\
\hline Model & 393.66 & 5 & 78.73 & 272.86 & 0.0003 & Significant \\
Number particles (A) & 127.59 & 1 & 127.59 & 442.18 & 0.0002 & Significant \\
Fluid Flowrate(B) & 213.07 & 1 & 213.07 & 738.43 & 0.0001 & Significant \\
AB & 0.015 & 1 & 0.015 & 0.053 & 0.8323 & Insignificant \\
A $^{2}$ & 30.38 & 1 & 30.38 & 105.29 & 0.0020 & Significant \\
B $^{2}$ & 22.61 & 1 & 22.61 & 78.35 & 0.0030 & Significant \\
Residual & 0.87 & 3 & 0.29 & & & \\
Cor Total & 394.52 & 8 & & & & \\
\hline
\end{tabular}

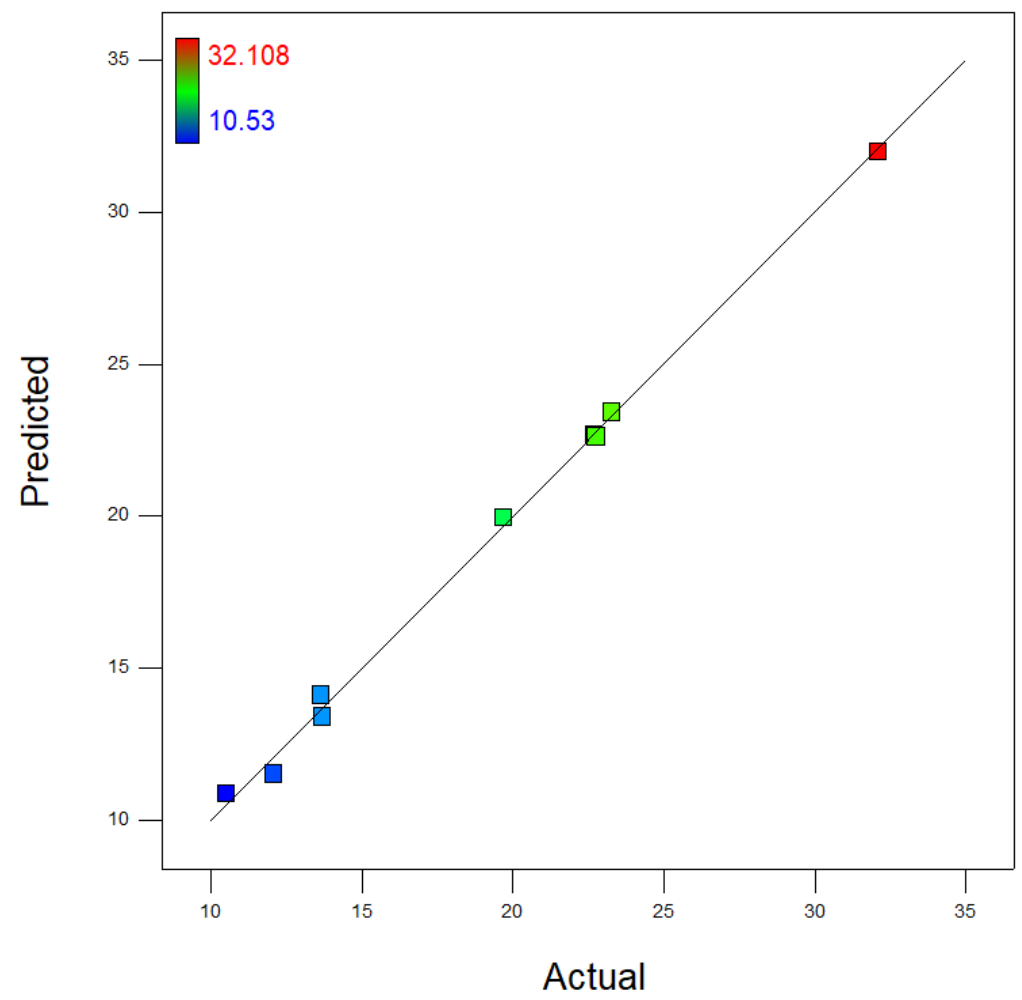

Figure 10: Model prediction as compared to actual results for $0.002 \mathrm{~m}$ particle fluidization.

Figure 11 presents the normal probability plot given by the RSM model. The viability of the statistical model can be evaluated by ensuring that the experimental error is normally distributed, as is the requirement for RSM. The normality of error distribution can be clearly seen in Figure 11, as no response transformation was required. Therefore, the normal probability plot supports the good estimate of the response, in the range of design variables studied. 


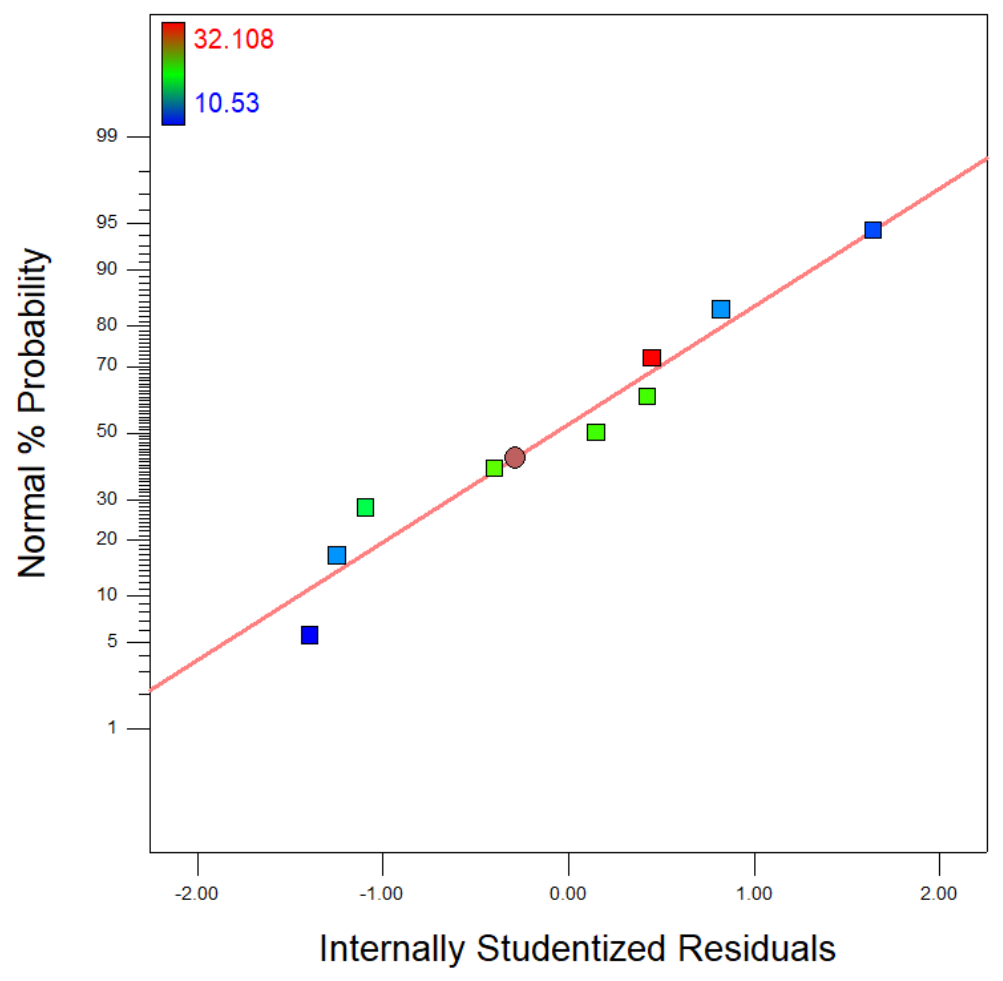

Figure 11: Normal probability plot of residuals.

A 3D surface plot, predicted by the statistical model, is presented in Figure 12. This surface plot is particularly useful for processes where an optimal BPVF is needed. When the number of particles increased at any specific fluid velocity, the BPVF increased. This was expected because the fluidized bed naturally became denser. Similarly, the BPVF decreased with increasing fluid velocity at any specific number of particles due to further upwards expansion of the bed. This further emphasizes the robustness of statistical methods with their ability to draw valuable information. The major advantage of statistical design of experiments, however, is that it can be used to potentially predict the BPVF for experiments which have not been performed, within the studied range of the design variables. 


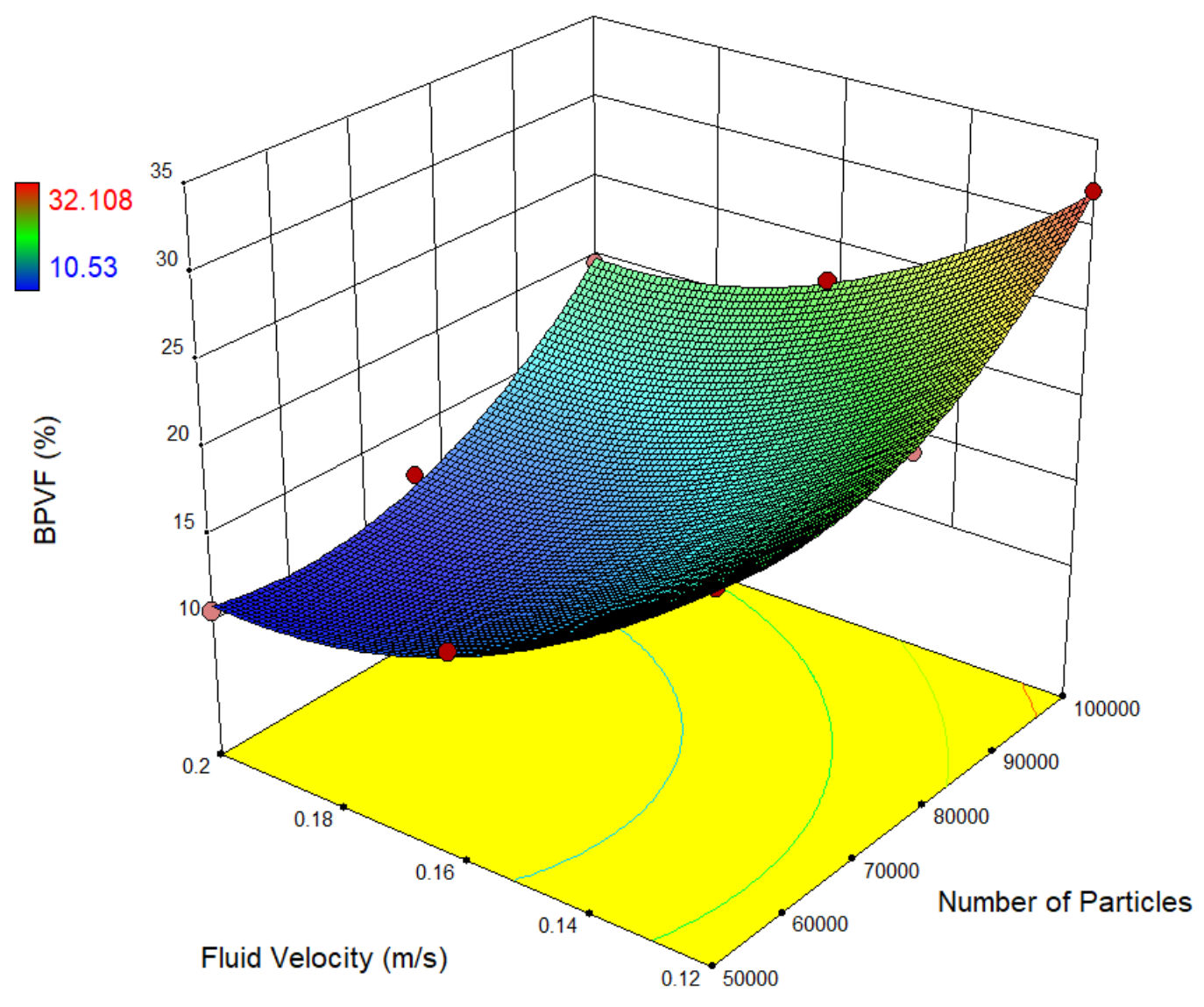

Figure 12: 3D Surface of the $0.002 \mathrm{~m}$ particle fluidization predicted model.

\subsection{Drag Force Analysis}

As the drag force is the dominant interaction force in liquid-solid fluidization (Zhu et al., 2007), initially it was attempted to analyze the influence of this force on LSFB behavior. In current literature of CFD-DEM simulations of LSFBs, the drag model is often chosen, and the influence of different drag models on liquid-solid fluidization has yet to be analyzed. Therefore, three common drag models were compared in this study: The Schiller-Naumann drag model, the Gidaspow drag model, and the Syamlal-O’brien drag model.

To test the influence of each drag model on simulation results, the percentage error (((experimental value - simulation value) /experimental value) $\times 100 \%$ ) was calculated for each simulation. All contact parameters were set as in Table 6. Moreover, no other interaction forces were added (i.e. no pressure gradient, virtual mass, or Saffman lift), and only drag force was considered. As 
previously mentioned, all simulations employed 50,000 particles of $0.002 \mathrm{~m}$ diameter fluidized at $0.16 \mathrm{~m} / \mathrm{s}$.

An experimental image and a snapshot of the simulation results are presented in Figure 13 and Figure 14, respectively. The maximum experimental bed height was observed to be $0.195 \mathrm{~m}$. It is worth mentioning that the bed height analysis was conducted based on the maximum bed height reached for all cases studied (repeated three times). It is clear from Figure 14a, that the SchillerNaumann drag model underestimated the fluidization behavior with $0.110 \mathrm{~m}$ bed height. Alternatively, the Syamlal-O'brien drag model (Figure 14c) overestimated the fluidization behavior with $0.38 \mathrm{~m}$ bed height. However, the Gidaspow drag model (Figure 14b) slightly overestimated the fluidization behavior, with $0.235 \mathrm{~m}$ bed height. Moreover, the Gidaspow drag model was able to replicate the instantaneous inclined particle arrangement at the bed surface seen experimentally from Figure 13. Therefore, among the studied drag models, the Gidaspow drag model provided the most accurate visual representation of experiments when compared to the Schiller-Naumann and Syamlal-O’brien drag models.

It can be seen, from Figure 13, that P2 was not fully covered by particles, however, the particles distinctly cleared the two bottom-most planes (P3 and P4). Therefore, to quantitatively compare the simulation and experimental results, the PPVF values of P3 and P4 were used, as was the case for the statistical model developed above.

Figure 15 shows a comparison between PPVF values obtained experimentally, and those estimated by simulations employing different drag models. As can be seen, the PPVF value predicted by the Schiller-Naumann drag model was considerably higher than experimental measurements obtained at $0.06 \mathrm{~m}$ (P4) with $110.46 \%$ difference, and considerably lower at $0.12 \mathrm{~m}$ (P3), with $98.18 \%$ difference. The observed deviation is a clear result of the gross underestimation of the bed height as seen in Figure 14a. The average percentage difference between the Schiller-Naumann drag model simulations and the experiments was $104.30 \%$.

Using the Syamlal-O'brien drag model, agreeable approximation of the PPVF was observed at $0.06 \mathrm{~m}$ height, with $10.61 \%$ difference. However, considerable deviation was observed at $0.12 \mathrm{~m}$, with $35.11 \%$ difference. This can be explained by the considerable overestimation of bed height when the Syamlal-O'brien drag model was employed in the simulations, as seen in Figure 14c. 
The average percentage error between the simulations using the Syamlal-O'brien drag model and experimental results was $22.86 \%$.

For simulations employing the Gidaspow drag model, an almost identical PPVF was noticed at $0.06 \mathrm{~m}$, when compared to experimental results, amounting to $2.24 \%$ difference. However, at 0.12 $\mathrm{m}$, a percentage error of $23.49 \%$ was observed, resulting in an average percentage error of $12.87 \%$. Though the deviation from experimental results can be attributed, in part, to the Gidaspow drag model slightly overestimating the experimental bed height (Figure 14b), this slight deviation can be due to the omission of interaction forces (other than drag), and due to the use of uncalibrated particle parameters initially employed. It has been shown that the omission of interaction forces (other than drag) causes liquid-solid fluidization behavior to resemble that of gas-solid systems by Malone et al. (2006). Also, the use of dry particle parameters in wet particle systems has been consistently shown to severely alter particle behavior in liquid-solid systems (Blais \& Bertrand, 2017; Ma et al., 2015). The justification of the omission of interaction forces, and the use of uncalibrated contact parameters, can be applied in full to the Syamlal O'brien and SchillerNaumann drag model simulations, however, significant deviation was witnessed when these two drag models were employed. The Gidaspow drag model is widely accepted in literature, and has been commonly used in CFD-DEM simulations of LSFBs (Liu et al., 2016; Peng et al., 2014, 2016). Overall, the data presented in Figures 13-15 demonstrate that the Gidaspow drag model produced results with the closest agreement with experiments in this study, and thus Gidaspow drag was selected for further analysis. 


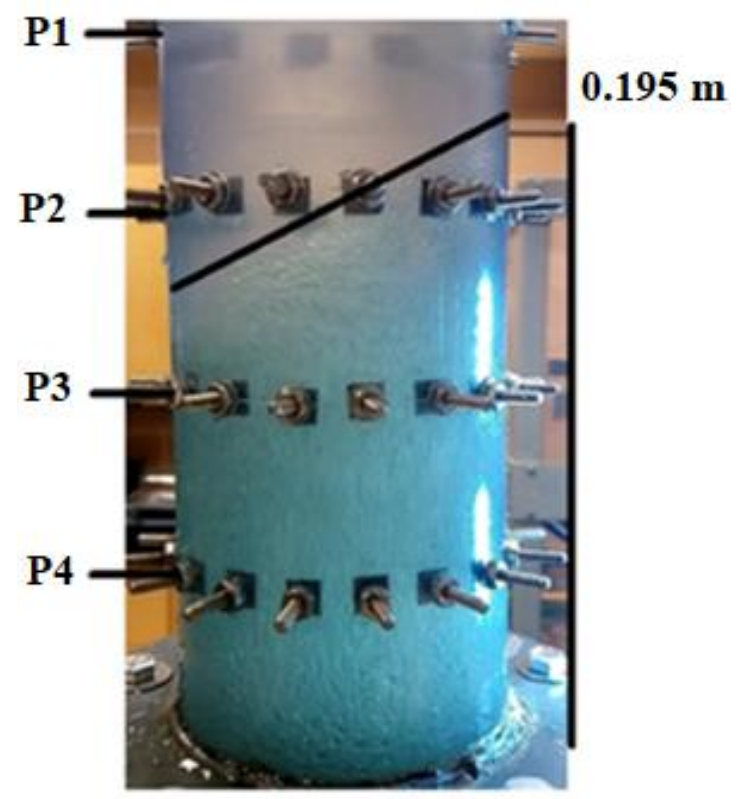

Figure 13: Experimental image of particle position during the fluidization of 50,000 particles fluidized at $0.16 \mathrm{~m} / \mathrm{s}$.

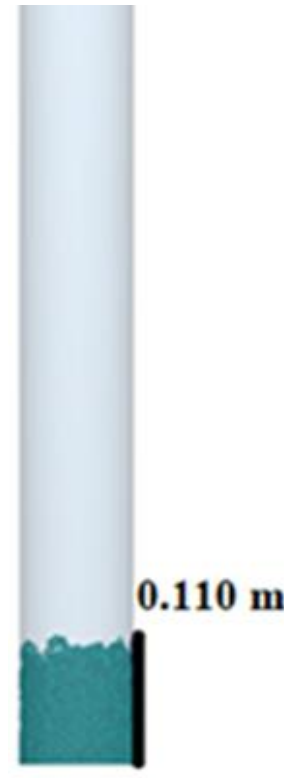

(a)

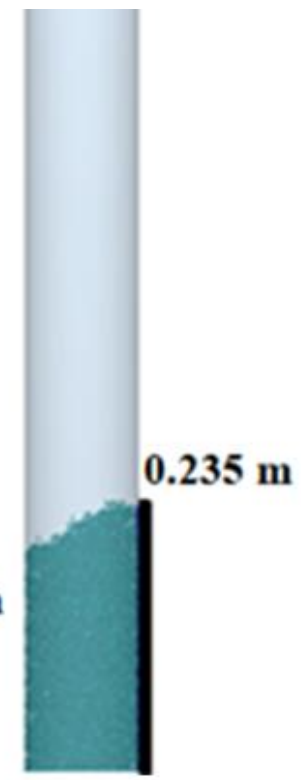

(b)

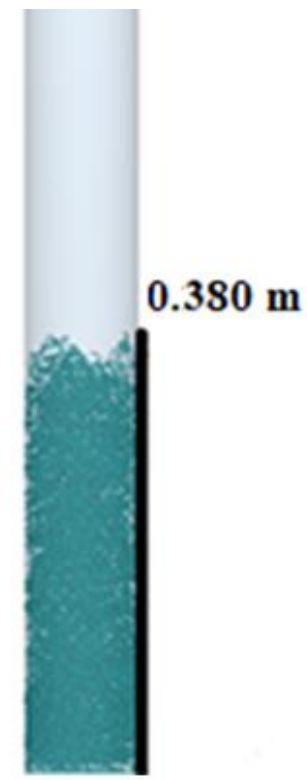

(c)

Figure 14: Comparison between fluidized bed height predicted by various drag models for 50,000, 0.002 $\mathrm{m}$ particles, at $0.16 \mathrm{~m} / \mathrm{s}$ a) Schiller-Naumann b) Gidaspow c) Symlal-O'brien. 


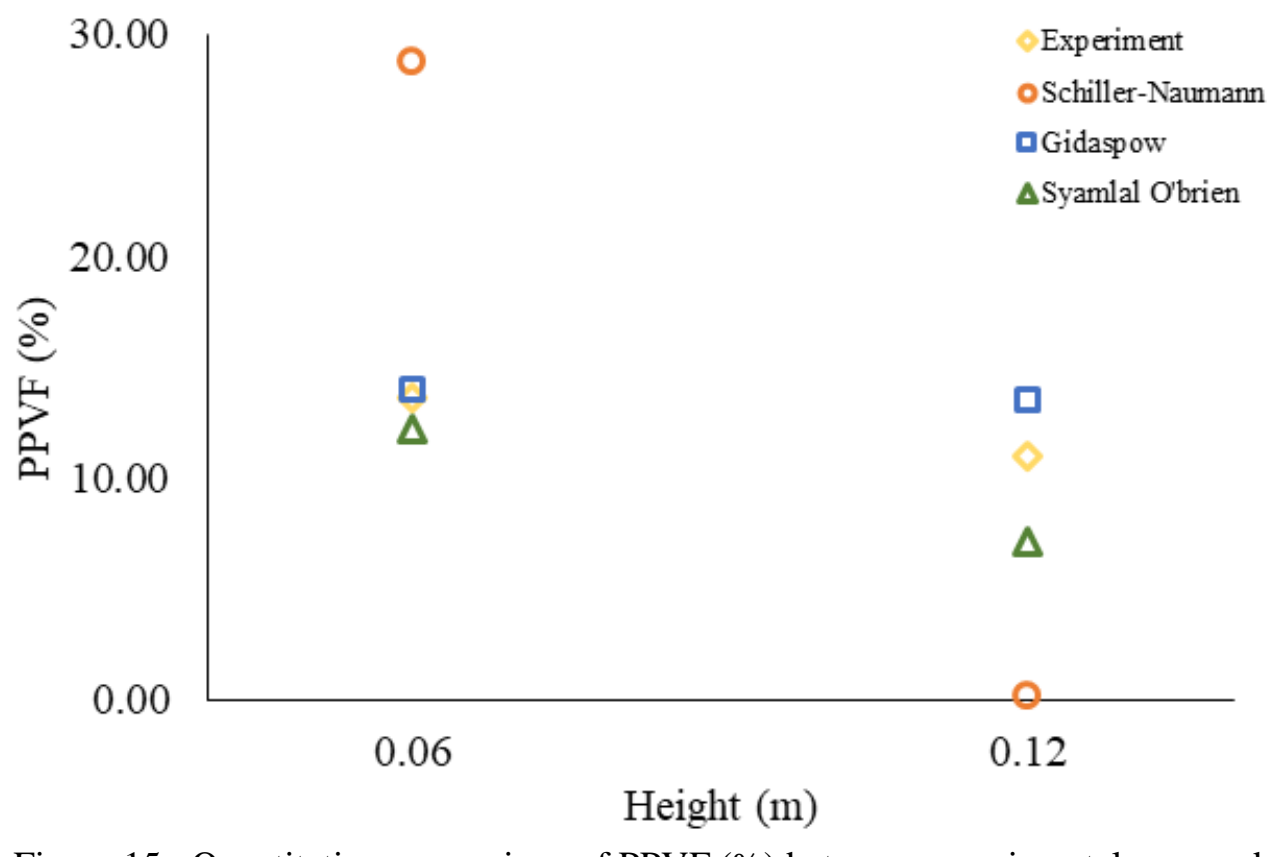

Figure 15: Quantitative comparison of PPVF (\%) between experimental runs and simulations using various drag models.

\subsection{Interaction Force Analysis}

In this section, the individual effect of interaction forces (other than drag) is quantified. Pressure gradient, virtual mass, and Saffman lift forces were added to investigate their individual influence on fluidized bed performance. Similar to drag model analysis, contact parameters were maintained as in Table 6. The simulation results of bed height and the percentage error for the addition of interaction forces are presented in Figure 16 and Figure 17, respectively. It is worth mentioning that the percentage error for the following sections represent the averaged percentage errors of both $0.06 \mathrm{~m}$ and $0.12 \mathrm{~m}$ planes.

The effect of pressure gradient on simulation results is presented in Figure 16b and Figure 17 . As can be seen in Figure 16b, the bed height reduced from $0.235 \mathrm{~m}$ to $0.230 \mathrm{~m}$ (Figure 16a to Figure 16b), compared to $0.195 \mathrm{~m}$ measured experimentally. Although the inclusion of pressure gradient force resulted in a minor reduction in percentage error, from $12.87 \%$ to $12.69 \%$, the decrease in bed height better estimated the experimental bed expansion and thus, the pressure gradient force was included in further analysis.

The effect of the inclusion of virtual mass force, alongside drag and pressure gradient forces, is presented in Figure 16c and Figure 17. Although no difference in bed height was observed in 
Figure 16c, the percentage error reduced from $12.69 \%$ to $10.30 \%$ (Figure 17). Virtual mass force accounts for the added mass the particles experience as they accelerate through a viscous fluid. The inclusion of this force may have caused the particles to re-arrange differently, which ultimately resulted in a closer match to experimental results. The significance of the inclusion of virtual mass force in liquid-solid fluidization was also observed by Ghatage et al. (2014), where the authors reported that the virtual mass had a dominant effect on the simulation results. In this study, the inclusion of virtual mass force reduced the percentage error by roughly $20 \%$. Therefore, the virtual mass force was included in further analysis.

The effect of the inclusion of Saffman lift force on simulation results is presented in Figure 16d and Figure 17. As can be seen in Figure 17, the addition of Saffman lift resulted in an increase in percentage error from $10.30 \%$ to $12.57 \%$. This can be attributed to a drastic increase in bed height from $0.230 \mathrm{~m}$ to $0.280 \mathrm{~m}$; vs. $0.195 \mathrm{~m}$ experimentally (As seen in Figure 16d). The inclusion of Saffman lift force in liquid-particle systems has been shown to greatly affect particle suspension in liquids (Blais \& Bertrand, 2017). However, in the current study this effect deteriorated the simulation accuracy. Thus, Saffman lift force was neglected from further analysis.

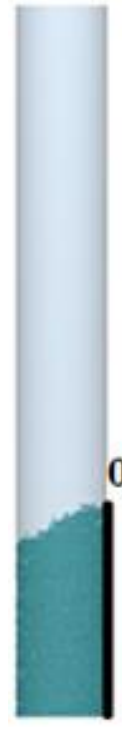

(a)

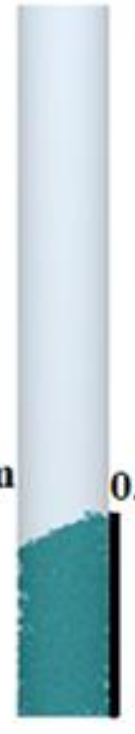

(b)

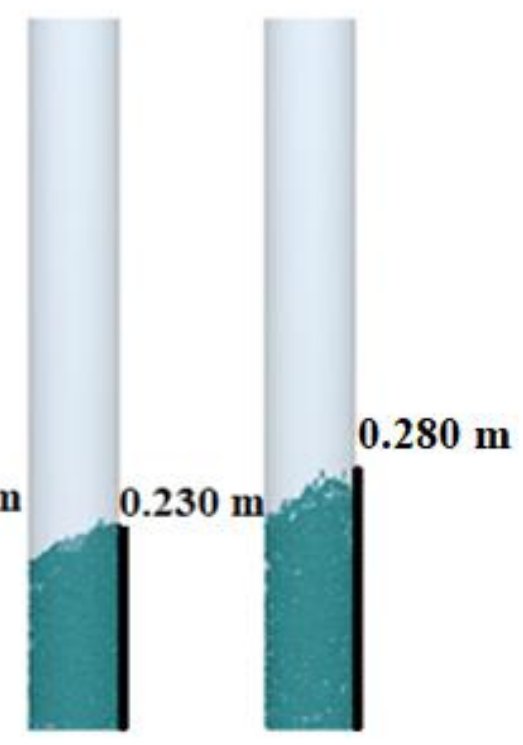

(c)

(d)

Figure 16: The influence of the inclusion of various interaction forces on simulation results (a) Gidaspow Drag Only (b) Drag + Pressure gradient (Pg) (c) Drag + Pg + Virtual mass (Vm) (d) Drag + Pg + Vm + Saffman lift (Saff). 


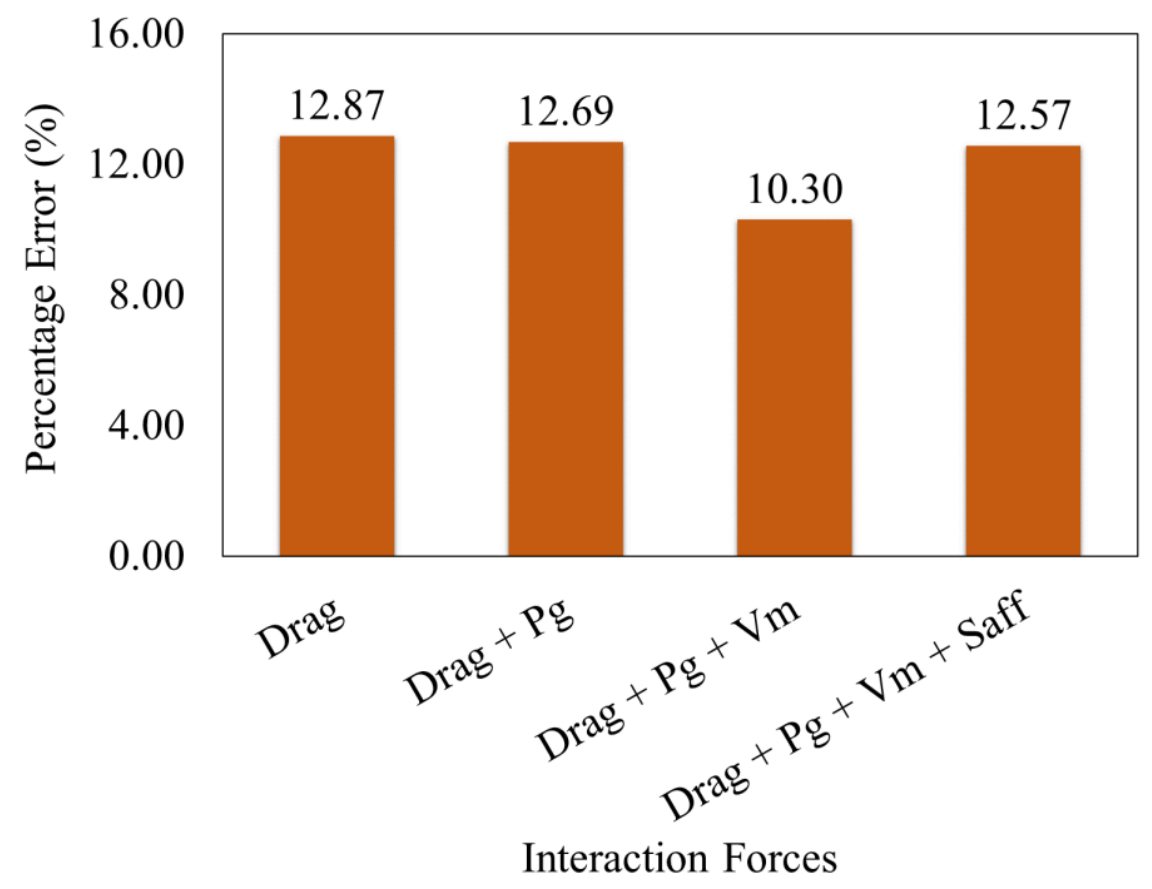

Figure 17: Percentage error pursuant to the addition of interaction forces.

The time-averaged magnitude of each interaction force is presented Table 10. As can be seen, the drag force accounts for almost all of the interaction force, which is also well documented in open literature (Al-Arkawazi et al., 2017; Sen et al., 2014; Zhu et al., 2007). Of interest is that the drag force is four orders of magnitude larger than pressure gradient and virtual mass forces. However, in this study, the inclusion of these two forces played a role in reducing the percentage error between the simulations and experiments. A similar result (qualitative only) was reported by Malone et al. (2006), where the authors showed that the inclusion of interaction forces (other than drag) improved the simulation results. Also, the virtual mass force is observed to be almost 4 times larger than the pressure gradient force. This may explain the minor reduction of percentage error observed in pressure gradient analysis $(12.87 \%$ to $12.69 \%)$, in comparison to the improvement seen in virtual mass force analysis (12.69\% to $10.30 \%$ ). Finally, the Saffman lift force magnitude was the largest of all interaction forces (other than drag), which explains its significantly higher effect on simulation results when compared to pressure gradient and virtual mass forces.

Table 10: Time-averaged magnitude of the interaction force.

\begin{tabular}{ccccc}
\hline Interaction force & Drag & Pressure Gradient & Virtual Mass & Saffman Lift \\
\hline $1.0258 \mathrm{E}-04 \mathrm{~N}$ & $1.0239 \mathrm{E}-04 \mathrm{~N}$ & $1.2800 \mathrm{E}-08 \mathrm{~N}$ & $4.8800 \mathrm{E}-08 \mathrm{~N}$ & $1.6200 \mathrm{E}-07 \mathrm{~N}$ \\
\hline
\end{tabular}




\subsection{Contact Parameter Analysis}

The time-averaged magnitude of the contact force (P-P and P-W) was found to be 4.99 E-05 N, which is almost half of that of the interaction force (Table 10). Since the interaction force was shown to greatly influence the simulation results in this study, the calibration of contact parameters was expected to produce a comparable improvement. In this section, the particle contact parameters are calibrated individually, initially with COR P-W, followed by COR P-P, $\mu_{\mathrm{s}} \mathrm{P}-\mathrm{W}, \mu_{\mathrm{s}}$ $\mathrm{P}-\mathrm{P}, \mu_{\mathrm{r}} \mathrm{P}-\mathrm{W}$, and finally, $\mu_{\mathrm{r}} \mathrm{P}-\mathrm{P}$. Once each contact parameter was calibrated, the calibrated value was used for all pursuant calibration simulations.

Table 11 presents the range of values for each contact parameter investigated in this study. The calibration method used by Blais \& Bertrand (2017) was applied, however, a different range of values were used in this study, as it was desired to investigate the effect of employing high, mid, and low values, of individual contact parameters, on simulation accuracy.

Table 11: Contact parameter calibration values

\begin{tabular}{cc}
\hline Contact Parameter & Range \\
\hline Coefficient of Restitution & $0.25,0.5,0.9$ \\
Coefficient of sliding friction & $0.1,0.3,0.9$ \\
Coefficient of rolling friction & $0.01,0.1,0.3$ \\
\hline
\end{tabular}

Initially, the coefficient of restitution (COR) P-W was varied from 0.25 , to 0.5 , and 0.9 . The remaining contact parameters were maintained as their initial values (Table 6). The results of varying COR P-W are presented in Figure 18a. As can be seen, by increasing the COR P-W to 0.5, the percentage error was reduced to $9.73 \%$. However, further increase of COR P-W to 0.9 increased the percentage error to $19.55 \%$. A high COR value (usually around 0.9 for glass beads in contact) is the classical 'dry' COR value for glass beads in air or vacuum, however, wet particles have been shown to behave with greater complexity than their dry counterparts (Gondret et al., 2002). The presence of a liquid between particles and particle-wall causes a transfer of kinetic energy from the particles to the liquid due to viscous dissipation (Gondret et al., 2002). Therefore, a lower COR is expected in wet systems. This was clearly observed in this study, as the percentage error was greatly increased by employing the dry COR P-W value. Alternatively, applying a very low COR P-W value had the opposite effect, as it resulted in higher viscous dissipation than was expected. This is observed when comparing Figure 18a for COR P-W 0.25 and 0.5 values. Since 
the percentage error was reduced by adjusting COR P-W value to 0.5 , this value was employed for further analysis.

COR P-P was varied from 0.9, to 0.25, and 0.5. As explained above, a COR value of 0.9 is representative of 'dry' particle contact. Therefore, reducing the COR P-P is expected to improve simulation results. Figure $18 \mathrm{~b}$ shows that calibrating the COR P-P value to 0.5 resulted in a decrease in percentage error from $9.73 \%$ to $6.51 \%$. It again verifies that the use of dry-dry contact parameters cannot be reliably applied to CFD-DEM simulations of LSFBs, and special attention must be paid when these parameters are selected for such simulations. Further reducing the COR P-P to 0.25 resulted in an increase in percentage error. As was explained above, higher than expected viscous dissipation led to a higher percentage error at 0.25 COR P-P. Thus, the calibrated COR P-P value of 0.5 was employed for further analysis.

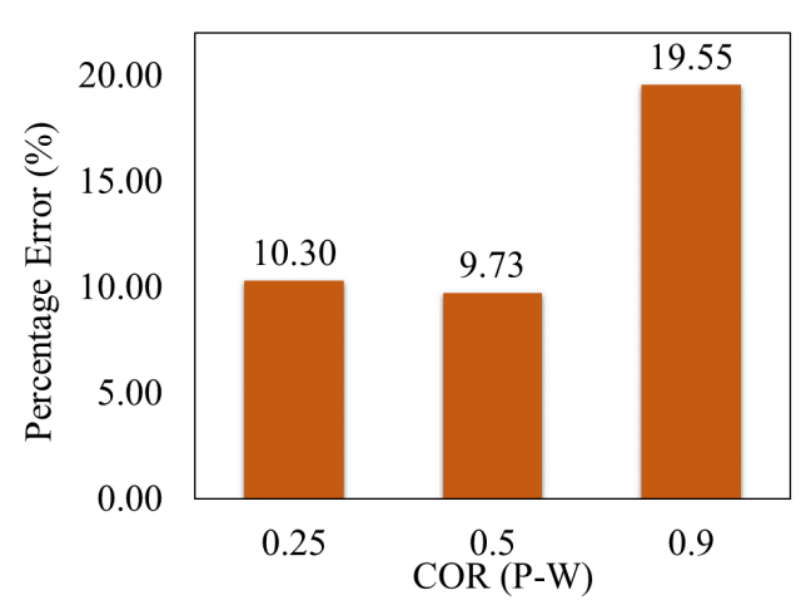

(a)

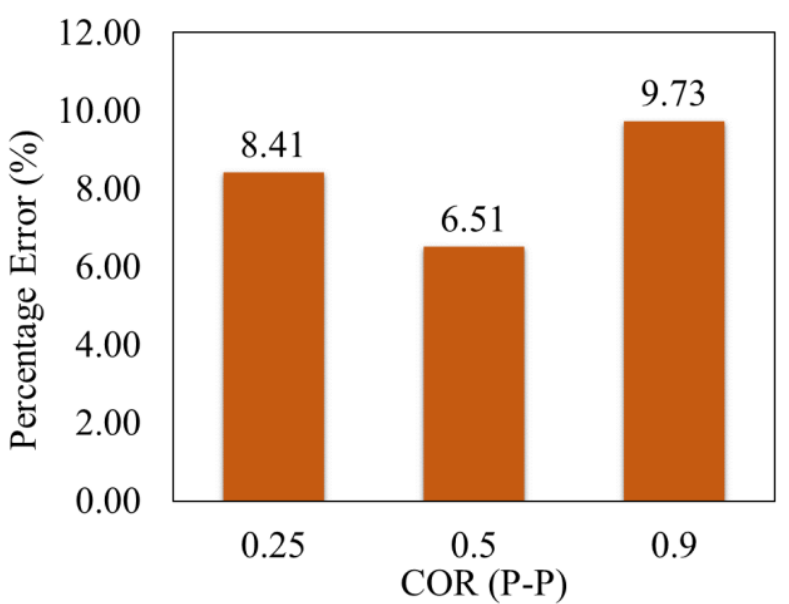

(b)

Figure 18: Coefficient of restitution calibration a) COR P-W b) COR P-P

To study the effect of $\mu_{\mathrm{s}}$ on simulation results, $\mu_{\mathrm{s}}$ was varied from 0.3 , to 0.1 , and 0.9 , for both P$\mathrm{W}$ and P-P contacts. Initially, the $\mu_{\mathrm{s}} \mathrm{P}-\mathrm{W}$ was calibrated, and the results are presented in Figure 19a. From the previous calibration steps (COR P-W and P-P), the percentage error between simulations and experiments was reduced to $6.51 \%$. Figure $19 \mathrm{a}$ shows that varying the initial $\mu_{\mathrm{s}} \mathrm{P}-$ $\mathrm{W}$ value of 0.3 increased the percentage error (for the range investigated). In liquid-particle systems, the liquid that covers the particle and geometry surfaces can act as a lubricant, reducing resistance to sliding motion (Wang et al., 2012). An increase in $\mu_{\mathrm{s}} \mathrm{P}-\mathrm{W}$ value implies a higher resistance to sliding motion, and applying this caused an increase in percentage error in this study. Alternatively, reducing the $\mu_{\mathrm{s}} \mathrm{P}-\mathrm{W}$ value to 0.1 unrealistically reduced the $\mathrm{P}-\mathrm{W}$ friction, 
consequently allowing particles to slide onto the wall with relative ease. Therefore, employing $\mu_{\mathrm{s}}$ $\mathrm{P}-\mathrm{W}$ value of 0.9 or 0.1 for PVC-glass contact in a water was concluded to be unrealistic, and thus, the $\mu_{\mathrm{s}} \mathrm{P}-\mathrm{W}$ value of 0.3 was maintained for further analysis.

Figure $19 \mathrm{~b}$ shows the effect of calibrating $\mu_{\mathrm{s}} \mathrm{P}-\mathrm{P}$ on simulation results. As can be seen, it was found that reducing $\mu_{\mathrm{s}} \mathrm{P}-\mathrm{P}$ to 0.1 , improved the percentage error to $4.74 \%$. Meanwhile, minimal differences were observed by increasing $\mu_{\mathrm{s}} \mathrm{P}-\mathrm{P}$ to 0.9 . The difference between the calibrated $\mu_{\mathrm{s}} \mathrm{P}-$ $\mathrm{P}(0.1)$ and $\mu_{\mathrm{s}} \mathrm{P}-\mathrm{W}(0.3)$ values was a direct consequence of the difference in the particles' and wall materials. Where there was a greater resistance to glass beads' ability to slide on a PVC surface, a lower resistance to sliding motion was observed between glass beads in contact with one another. An increase in $\mu_{\mathrm{s}} \mathrm{P}-\mathrm{P}$ to 0.9 was also unrealistic since a high $\mu_{\mathrm{s}} \mathrm{P}-\mathrm{P}$ value implied that particles adhered to one another, which was clearly not the case in this study. Since employing the $\mu_{\mathrm{s}} \mathrm{P}-\mathrm{P}$ value of 0.1 resulted in the least percentage error, this value was used for further analysis.

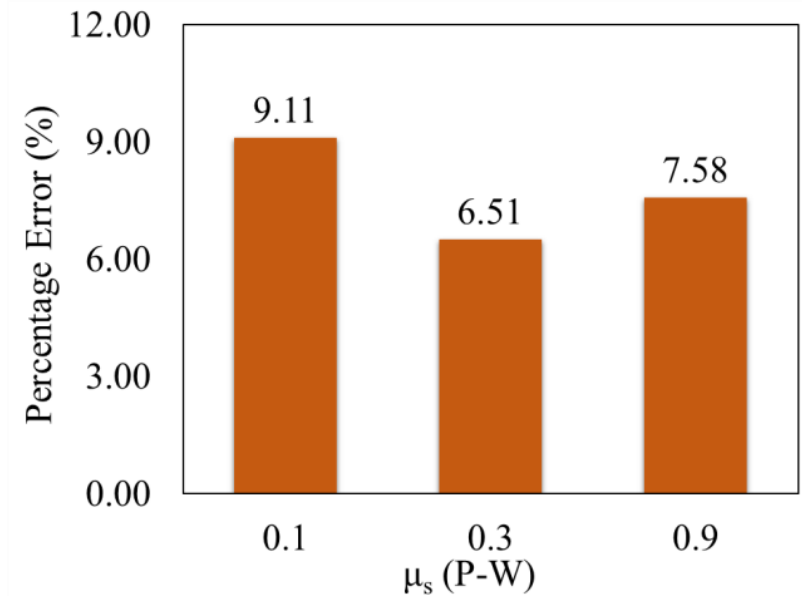

(a)

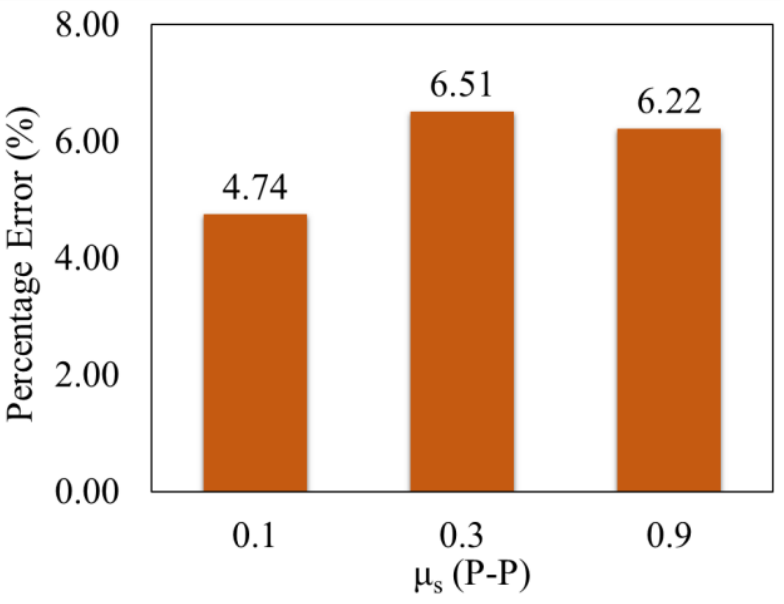

(b)

Figure 19: Coefficient of sliding friction calibration a) $\mu_{\mathrm{s}} \mathrm{P}-\mathrm{W}$ a) $\mu_{\mathrm{s}} \mathrm{P}-\mathrm{P}$

The effect of $\mu_{\mathrm{r}}$ on simulation results was investigated by varying $\mu_{\mathrm{r}}$ from 0.1 , to 0.01 and 0.3 , for both P-W and P-P contacts. As can be seen from Figure 20a, reducing the $\mu_{\mathrm{r}} \mathrm{P}-\mathrm{W}$ value to 0.01 resulted in an increase in the percentage error. This observation can be described by the presence of an interstitial liquid, which causes damping in particle rotation (Blais \& Bertrand, 2017). This corresponds to an increased rolling friction value. Therefore, selecting a low value of 0.01 for $\mu_{\mathrm{r}}$ cannot represent the reality. Also, as can be seen in Figure 20a, increasing the $\mu_{\mathrm{r}}$ value to 0.3 further increased the percentage error between simulations and experiments. A $\mu_{\mathrm{r}}$ value of 0.3 implies that the particles' ability to roll on the surface is hindered to a greater degree than expected. Therefore, 
employing 0.01 or $0.3 \mu_{\mathrm{r}} \mathrm{P}-\mathrm{W}$ values were concluded to be unrealistic, and thus, the $\mu_{\mathrm{r}} \mathrm{P}-\mathrm{W}$ value was maintained at 0.1. Similar results are observed in Figure $20 \mathrm{~b}$ for $\mu_{\mathrm{r}} \mathrm{P}-\mathrm{P}$ contact. Increasing or reducing the initial $\mu_{\mathrm{r}} \mathrm{P}-\mathrm{P}$ value (i.e. 0.1) again resulted in an increase in percentage error, and the reasoning mentioned for the calibration of $\mu_{\mathrm{r}} \mathrm{P}-\mathrm{W}$, also applies to calibration of P-P rolling friction. This further confirms that dry particles behave differently when wetted.

The final, calibrated contact parameter values are presented in Table 12. A summary of the effect of interaction force analysis, and contact parameters calibration on the percentage error is presented in Figure 21. This Figure shows the selected drag model, the relevant interaction forces, and the calibrated contact parameter values leading to the closest agreement between simulation and experimental results.

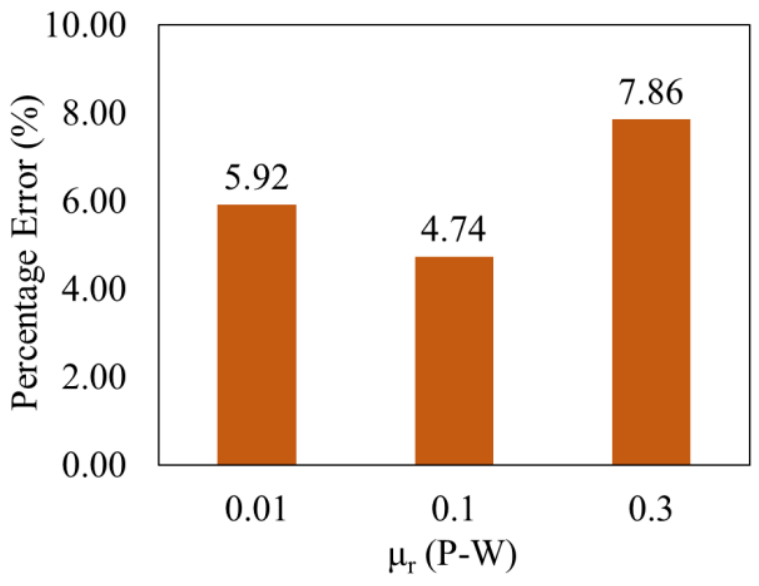

(a)

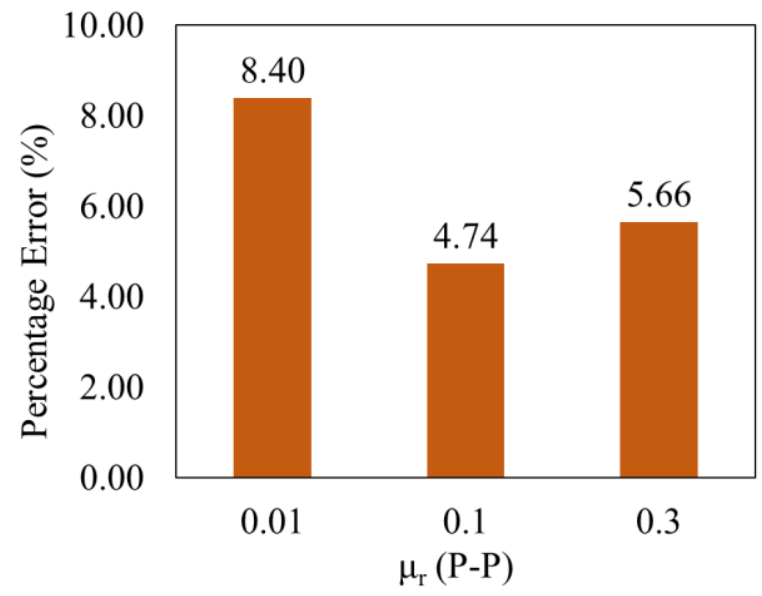

(b)

Figure 20: Coefficient of rolling friction calibration a) $\mu_{\mathrm{r}} \mathrm{P}-\mathrm{W}$; b) $\mu_{\mathrm{r}} \mathrm{P}-\mathrm{P}$

Table 12: Final calibrated particle parameter values

\section{Contact Parameters}

Coefficient of Restitution (P-W)

Coefficient of sliding friction $(\mathrm{P}-\mathrm{W})$

Coefficient of rolling friction $(\mathrm{P}-\mathrm{W})$
0.5 (P-W), 0.5 (P-P)

0.3 (P-W), 0.1 (P-P)

$0.1(\mathrm{P}-\mathrm{W}), 0.1(\mathrm{P}-\mathrm{P})$ 


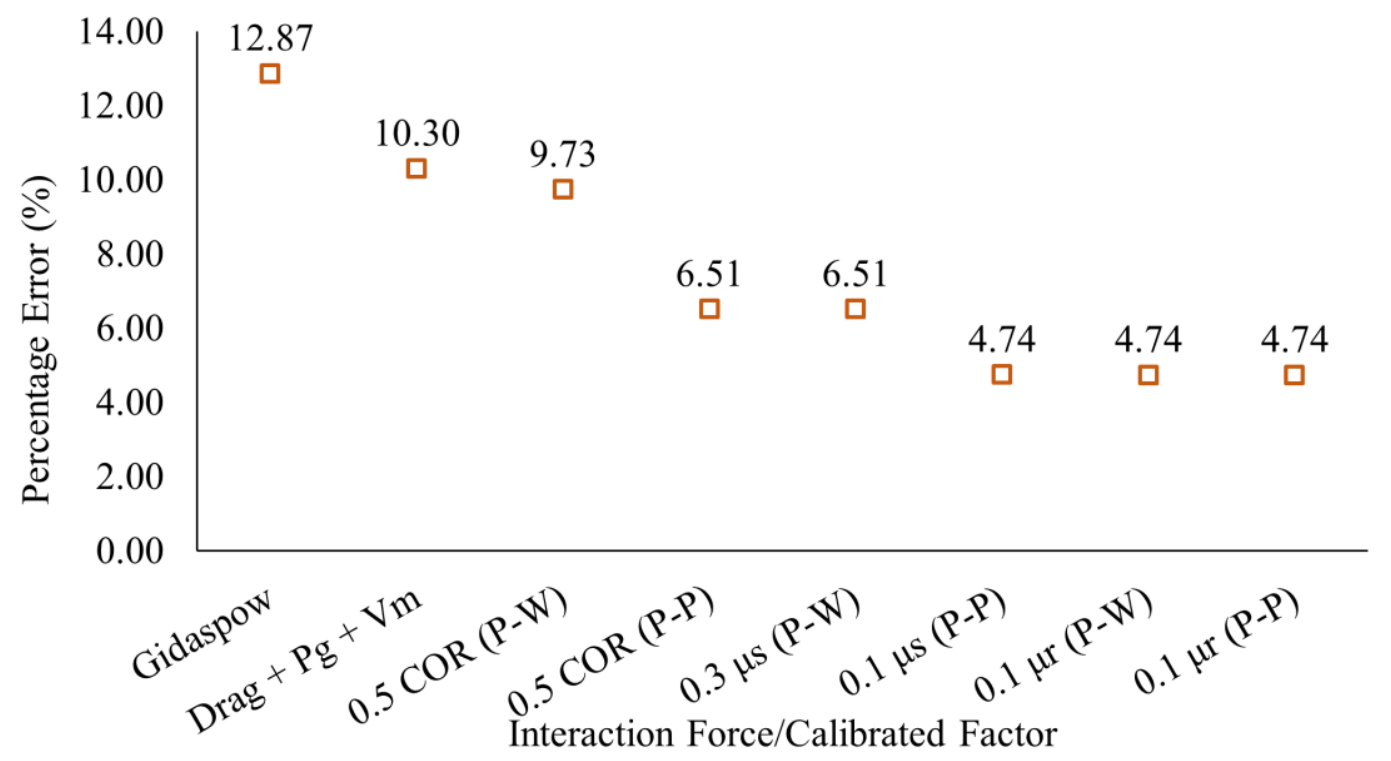

Figure 21: Summary of interaction force analysis and contact parameters calibration. 


\subsection{Local Comparison between Simulation and Experimental Results}

So far, the comparison between simulation and experimental results has been conducted on a global value: the PPVF. This required the temporal and spatial averaging of the particle volume fraction (PVF). Spatial averaging can be problematic as it cannot represent the particle distribution along the plane. Therefore, in this section, the comparison between the simulation and the experiment $(50,000$ particles of $0.002 \mathrm{~m}$ diameter fluidized at $0.16 \mathrm{~m} / \mathrm{s})$ using the combination of the Gidaspow drag, pressure gradient force, virtual mass force, and the calibrated contact parameters was redone to analyze the radial distribution of particles obtained through simulations and ERT experiments. This will provide a starting point to studying the hydrodynamic behavior of the flow regime encountered at various operating conditions (i.e. fluid superficial velocities and number of particles).

Figure 22 shows the experimental measurements and simulation results of the time-averaged particle volume fraction (PVF) at $0.12 \mathrm{~m}$ height, along the bed's radius $\left(0^{\circ}\right.$ angle), for 50,000 particles fluidized at $0.16 \mathrm{~m} / \mathrm{s}$. It is worth mentioning that the spatial resolution of the tomography system is about $\pm 5 \%$ of the column diameter. As observed, the average percentage error between the simulation and experiments was $3.89 \%$, indicating that the simulation and experimental results were within close agreement. As can be seen, the experimental PVF increased minimally from the center of the bed towards the wall. The PVF obtained from the simulation matched the increasing trend near the center of the bed, slightly overestimating the PVF values, and exhibited a minor decreasing trend near the wall, with a slight underestimation. The slight overestimation of experimental data in the central section of the LSFB, may be explained by the choice of the drag model. Peng et al. (2016) and Wang et al. (2012) employed the Gidaspow drag model and observed similar overestimation of results. However, the slight discrepancy between experimental and simulation results near the wall can be attributed to the inherent limitation of the CFD-DEM method in which near-wall regions mesh size cannot be resolved finely (i.e. the mesh size must be larger than the particle diameter). Therefore, the fluid flow near the wall cannot be modelled precisely (Ebrahimi, 2014). Wang et al. (2012) also observed considerable deviation from experimental data of PVF values near the wall, with roughly $16 \%$ difference. The authors mainly attributed this difference to the loss of 1 dimensional information, as their simulations were conducted in $2 \mathrm{D}$ geometry. In this study however, the simulation results were very close to the 
experimental values and were within the experimental data standard deviation range. Comparable results were observed at $0.06 \mathrm{~m}$ bed height.

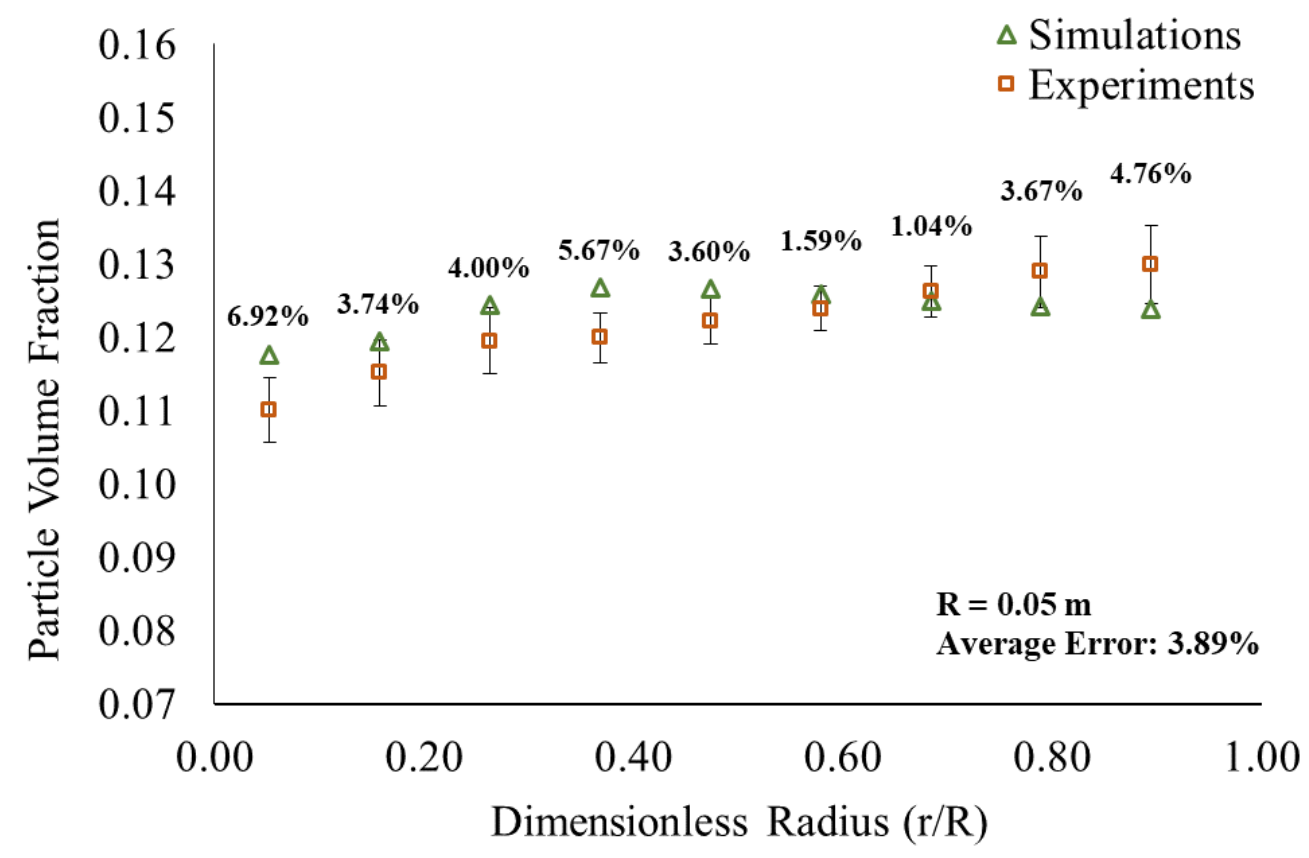

Figure 22: Comparison between experimental and simulation PVF in the radial direction $\left(0^{\circ}\right.$ angle) at $0.12 \mathrm{~m}$ height, for 50,000 particles at $0.16 \mathrm{~m} / \mathrm{s}$. (Percentages above the data points

represent the percentage error between simulations and experiments at the prevailing dimensionless radius).

\subsection{The Influence of Liquid Superficial Velocity on System Hydrodynamics}

In this section, the Wallis (1962) stability criterion and CFD-DEM results were applied to identify and analyze the flow regime at various liquid superficial velocities. Furthermore, the effect of liquid superficial velocity on liquid and particle velocity profiles and PVF was also quantified. The liquid superficial velocity was varied from $0.12 \mathrm{~m} / \mathrm{s}$ to $0.16 \mathrm{~m} / \mathrm{s}$ and $0.20 \mathrm{~m} / \mathrm{s}$, while maintaining the number of particles constant at 50,000.

\subsubsection{Stability Analysis for Various Liquid Superficial Velocities}

Figure 23 shows instantaneous snapshots of the fluidization of 50,000 particles at $0.12 \mathrm{~m} / \mathrm{s}, 0.16$ $\mathrm{m} / \mathrm{s}$, and $0.20 \mathrm{~m} / \mathrm{s}$. As expected, the bed height increased with increasing liquid superficial velocity. For $0.12 \mathrm{~m} / \mathrm{s}$ fluidization, the bed was observed to be very dense in comparison to $0.16 \mathrm{~m} / \mathrm{s}$ and $0.20 \mathrm{~m} / \mathrm{s}$ fluidization, and minimal fluctuations in bed height were observed. As the liquid superficial velocity was increased to $0.16 \mathrm{~m} / \mathrm{s}$, an increase in chaotic behavior was observed. Furthermore, greater fluctuations in bed height were observed for $0.20 \mathrm{~m} / \mathrm{s}$ fluidization in comparison to those for $0.16 \mathrm{~m} / \mathrm{s}$. 


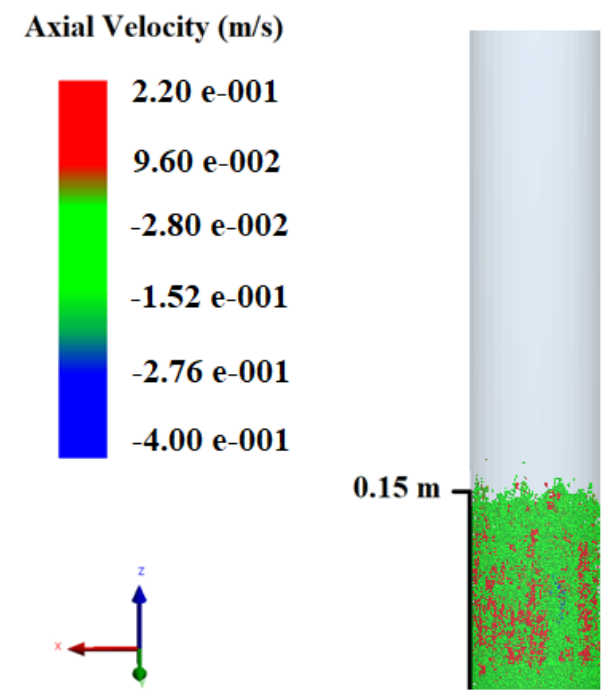

(a)

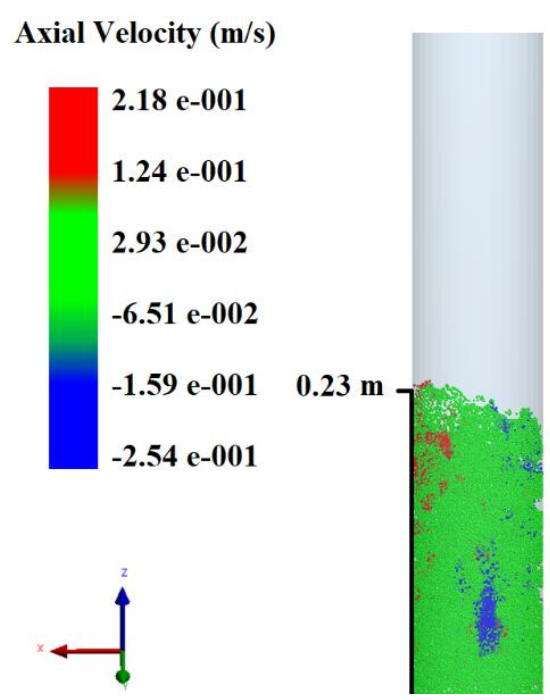

(b)

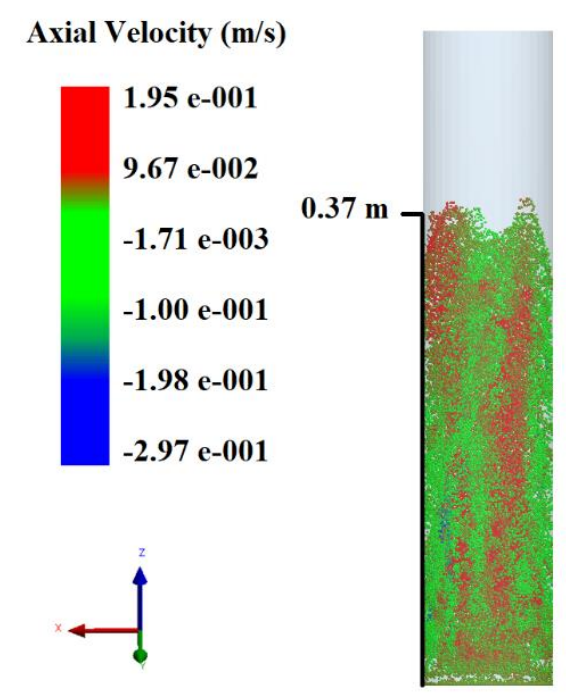

(c)

Figure 23: Instantaneous snapshots of 50,000 particles fluidized at a) $0.12 \mathrm{~m} / \mathrm{s} \mathrm{b}) 0.16 \mathrm{~m} / \mathrm{s}$ and c) 0.20 $\mathrm{m} / \mathrm{s}$.

Figure 24 shows the Wallis (1962) stability criterion for $0.002 \mathrm{~m}$ glass beads fluidized with water as a function of bed porosity. As seen, the stability values were positive (implied homogeneity) regardless of bed porosity. However, as stated by Gibilaro (2001), intermediate stability can be encountered for fluidization of $0.002 \mathrm{~m}$ glass beads with water, since in general the stability criterion values are relatively close to zero. The colored data points in Figure 24 represent the bedaveraged porosity calculated from the CFD-DEM results (averaged over 10 seconds of simulation) 
for the liquid superficial velocities studied. As can be seen, the stability increased with increasing liquid superficial velocity (for the range tested).

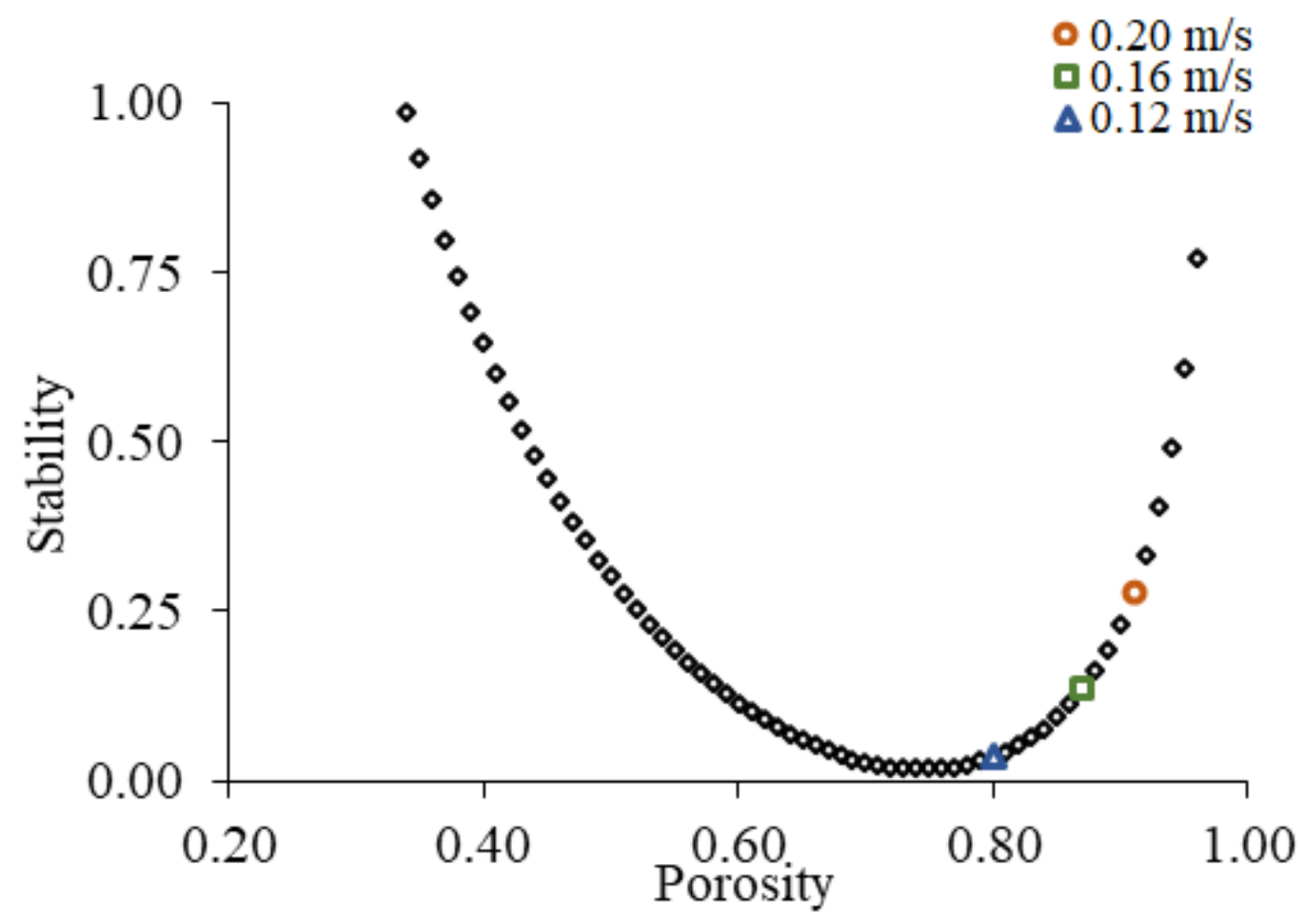

Figure 24: Stability as a function of porosity for $0.002 \mathrm{~m}$ glass beads fluidized with water. Porosities calculated from the CFD-DEM results at various flowrates are indicated by colored points $(50,000$ particles).

To further analyze the flow regime for various liquid superficial velocities, the PVF profiles along the bed height obtained through CFD-DEM simulations is shown in Figure 25. This approach was previously used by Peng et al. (2016) and Wang et al. (2012). As can be seen for $0.12 \mathrm{~m} / \mathrm{s}$ fluidization, the change in PVF along the bed height exhibited a slightly irregular trend where the PVF values near the middle of the bed were higher than near the entrance and close to the top. The Wallis (1962) stability criterion predicted a stability value very close to zero (stability limit, Figure 24) at this condition, which can explain the observed PVF behavior. This was a strong indication that the intermediate flow regime was produced at this liquid superficial velocity. At $0.16 \mathrm{~m} / \mathrm{s}$, stability analysis showed a slight increase in stability when compared to $0.12 \mathrm{~m} / \mathrm{s}$ (Figure 24). This translated into the disappearance of the irregularity seen in Figure 25 for $0.12 \mathrm{~m} / \mathrm{s}$, however, a slight decrease in PVF was observed along the bed height. Since the PVF varied along the bed height (the homogeneous flow regime is characterized by a constant PVF along the bed height) 
and bubbling behavior was not observed at this condition, the flow regime can be classified as intermediate at $0.16 \mathrm{~m} / \mathrm{s}$. At $0.20 \mathrm{~m} / \mathrm{s}$, an increase in stability was observed in comparison to 0.16 $\mathrm{m} / \mathrm{s}$ fluidization of 50,000 particles (Figure 24). The PVF trend at this condition exhibited a relatively minor decrease along the bed height in Figure 25, and thus the trend cannot be confidently assumed to be constant and complete homogeneity may be doubtful.

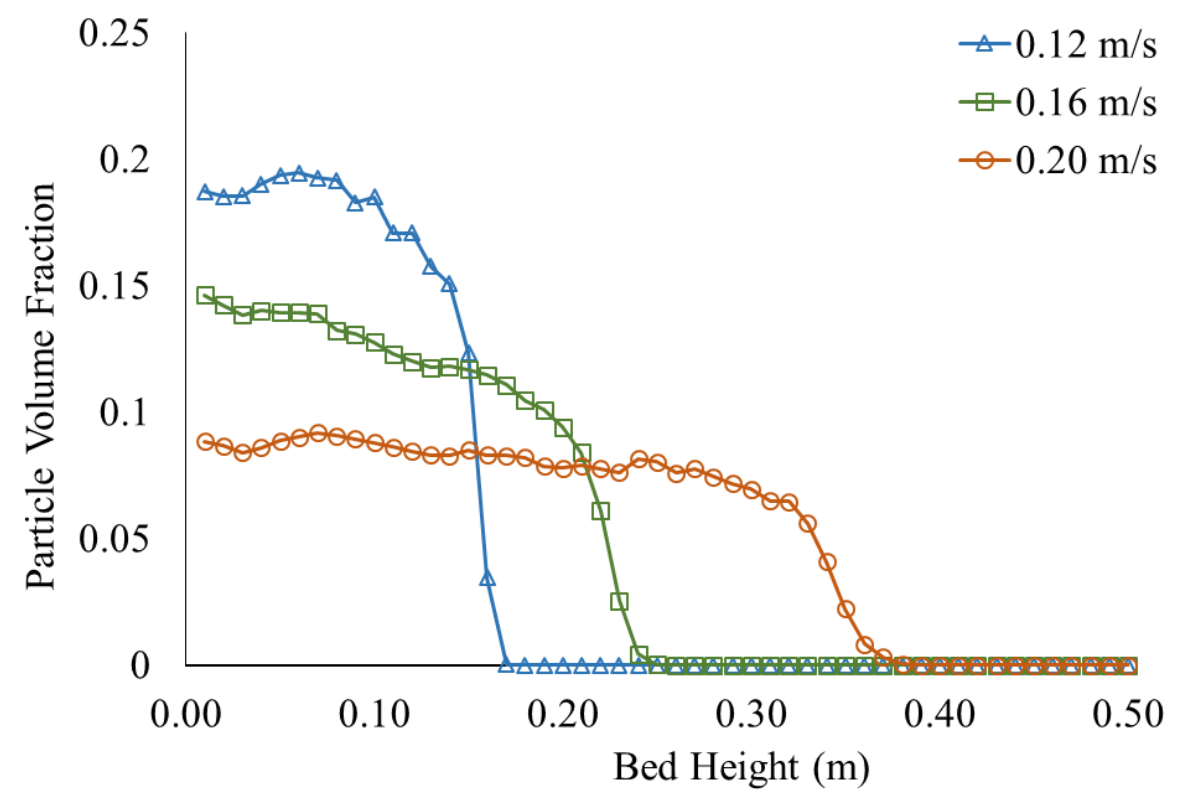

Figure 25: PVF variation along the bed height at various liquid superficial velocities.

To further analyze the flow regime at $0.20 \mathrm{~m} / \mathrm{s}$, the particle velocity vectors at various simulation times are presented in Figure 26. Figure 26 shows two distinct hydrodynamic behaviors: the formation of vortices and gulf-streams (Di Renzo et al., 2011; Epstein, 2003). The vortices appeared at random locations throughout the LSFB as time progressed. However, the gulf streams, solely appeared at two distinct locations slightly off-center; although they may be absent at certain time-steps, and present at others. Studying a 2D LSFB, with predominantly homogeneous flow regime, Di Renzo et al. (2011) explained that homogeneity occurs due to very well-mixed behavior within the LSFB, characterized by locally displacing vortices, similar to what was observed in Figure 26. On the other hand, Epstein (2003) stated that the gulf-streaming phenomenon can be an indication of non-homogeneous flow behavior in LSFBs. Therefore, due to the formation of vortices (contributes to homogeneity) and gulf-streaming (contributes to non-homogeneity) and the clear lack of bubbling behavior (characteristic of fully unstable beds), it can be concluded that the fluidized bed at $0.20 \mathrm{~m} / \mathrm{s}$ also exhibited the intermediate flow regime. 
The gulf-streaming phenomenon was also observed for conditions at $0.16 \mathrm{~m} / \mathrm{s}$, however, the location of the appearance (and disappearance, as it alternates) of gulf-streams was different. Whereas for $0.20 \mathrm{~m} / \mathrm{s}$ two gulf-streams were observed in the off-center, for $0.16 \mathrm{~m} / \mathrm{s}$ a single gulfstream near the center was encountered. This is illustrated in Figure 27. This figure shows the time-averaged liquid velocity contour along the bed height at $0.16 \mathrm{~m} / \mathrm{s}$ and $0.20 \mathrm{~m} / \mathrm{s}$. As seen in Figure 27a, there existed a higher velocity region near the center confirming the single central gulfstream. Furthermore, for $0.20 \mathrm{~m} / \mathrm{s}$, the highest velocity was observed in two locations off-center, thus confirming the two previously discussed gulf-streams. 


\section{— Gulf Streaming \\ - Vortex}

Velocity (m/s)

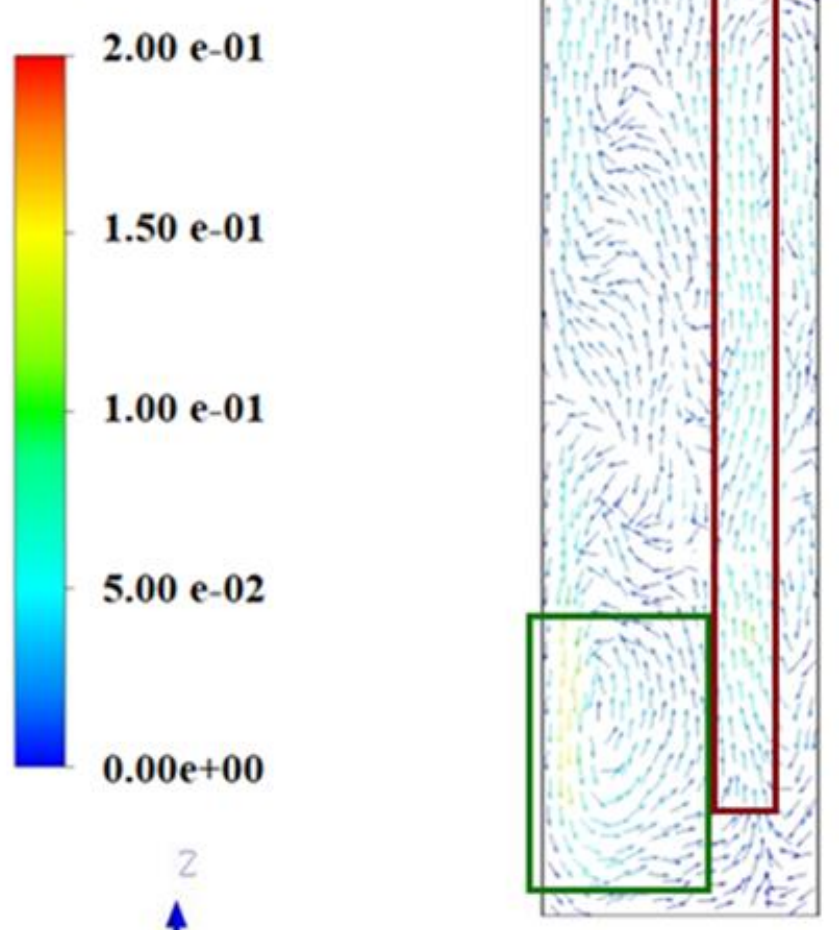

$12.0 \mathrm{~s}$

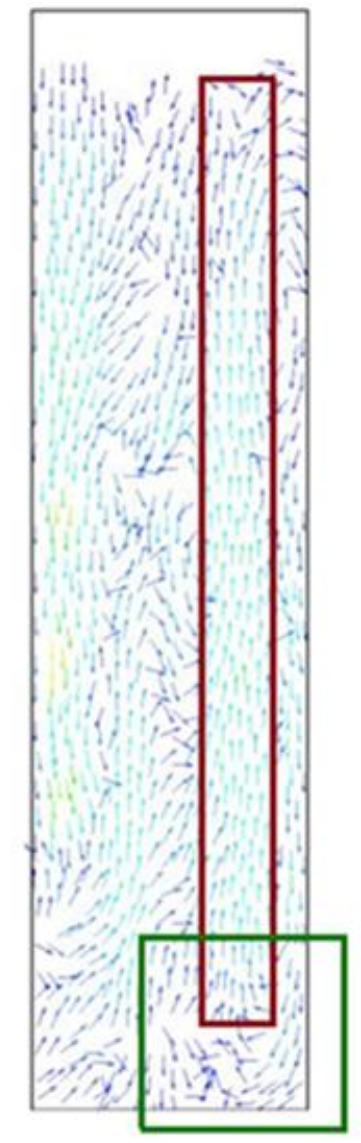

$13.0 \mathrm{~s}$

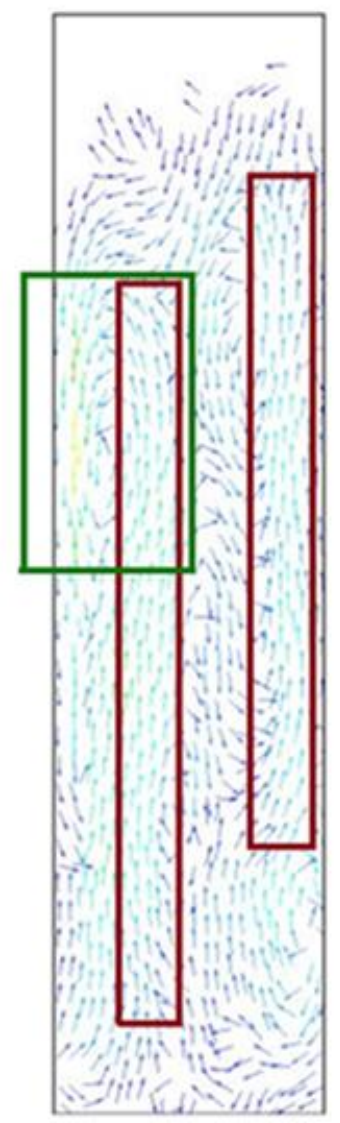

$14.0 \mathrm{~s}$

Figure 26: Particle velocity vectors at various simulation time. 


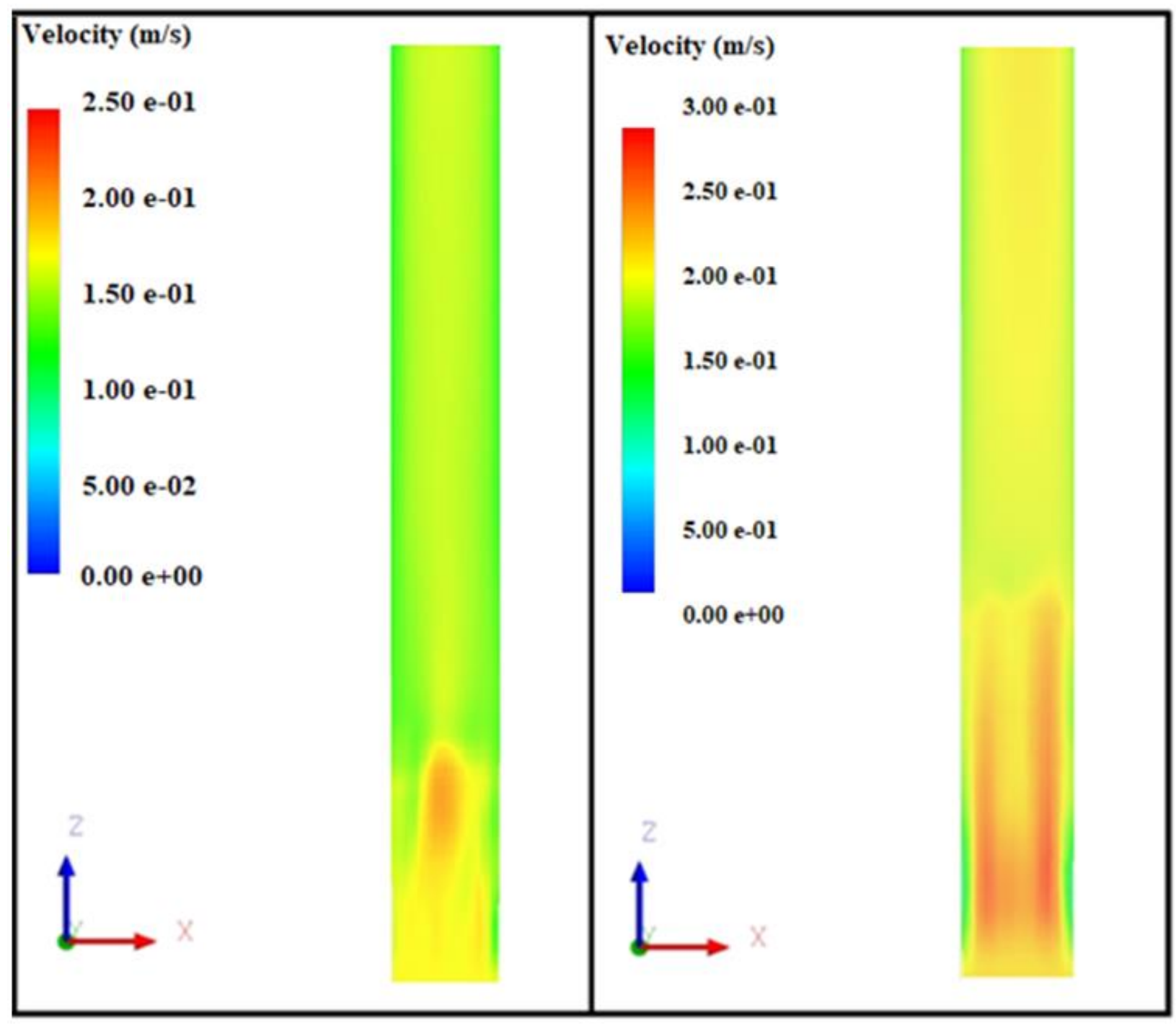

(a)

(b)

Figure 27: Time-averaged liquid velocity contour along the bed height with 50,000 particles. a) $0.16 \mathrm{~m} / \mathrm{s}$ c) $0.20 \mathrm{~m} / \mathrm{s}$.

\subsubsection{The Effect of Liquid Superficial Velocity on the Liquid and Particle Flow}

\section{Behavior}

In this section, the effect of the liquid superficial velocity on liquid and particle flow behaviors was evaluated by quantifying the liquid and particle mean axial velocities (MAVs) and PVF along the radial direction at $0.12 \mathrm{~m}$ height. The liquid and particle MAVs and PVF were averaged over $10 \mathrm{~s}$ proceeding the visual confirmation of the onset of steady state conditions.

Figure 28 shows the liquid (a) and particle (b) MAVs, and PVF (c) at $0.12 \mathrm{~m}$ for simulations including 50,000 particles fluidized at various liquid superficial velocities. As expected, the liquid MAV increased with increasing liquid superficial velocity (Figure 28 a). As can be seen in Figure 
28a, the liquid velocity profile at $0.12 \mathrm{~m} / \mathrm{s}$ was slightly asymmetrical and irregular in trend. This can be explained by the near zero stability predicted by the Wallis (1962) criterion at this condition (section 4.6.1). This figure further exhibited a single slightly asymmetrical peak near the center for fluidization at $0.16 \mathrm{~m} / \mathrm{s}$. Similar trend (single peaks in axial velocity) has been previously reported experimentally by Limtrakul et al. (2005) for the fluidization of $0.002 \mathrm{~m}$ glass beads with water; however, the flow regime was not discussed. As can be seen at $0.20 \mathrm{~m} / \mathrm{s}$ liquid superficial velocity, the liquid MAV showed two relatively symmetrical off-center peaks. The two off-center peaks were a direct result of the gulf-streaming effect discussed in section 4.6.1.

For 0.16 and $0.20 \mathrm{~m} / \mathrm{s}$, the particle MAV (Figure 28b) followed identical trends to the liquid MAV at $0.12 \mathrm{~m}$ height. Di Renzo et al. (2011) explained that particle motion is fully governed by that of the fluid, and proved their results for the homogeneous flow regime. Results in this study also showed that for 0.16 and $0.20 \mathrm{~m} / \mathrm{s}$ fluidizations in the intermediate flow regime, the particles' motion also follows that of the liquid. Generally, for $0.20 \mathrm{~m} / \mathrm{s}$ the particles fell downwards in regions close to the wall, and flowed upwards in the remainder of the fluidized bed. However, for $0.16 \mathrm{~m} / \mathrm{s}$ the particles moved upwards only close to the center, and downwards throughout the rest of the bed. Similar circulation patterns (upwards near the center and downwards near the wall) were reported by Wang et al. (2012) and Liu et al. (2016) when applied the CFD-DEM approach to simulate a 2D LSFB operating in homogeneous regime. For $0.12 \mathrm{~m} / \mathrm{s}$ fluidization, however, it cannot be confidently concluded that the particles MAV have followed an identical trend to that of the liquid. The circulation pattern exhibited a highly irregular trend, where on average, the particles possessed positive MAVs near the center of one side of the bed, however constant particle MAV on the opposite side of the bed, and negative particle MAV near the wall. The irregularity can be explained by the fluidization behavior at these conditions where the bed was shown to be i) near the stability limit (zero), and ii) very dense, with minimal particle movement. As can be seen, the lowest stability case $(0.12 \mathrm{~m} / \mathrm{s})$ exhibited the least amount of symmetricity in particle flow behavior. Although the particle MAVs (Figure 28b) appeared to be comparable at $0.12 \mathrm{~m} / \mathrm{s}$ and $0.16 \mathrm{~m} / \mathrm{s}$ liquid superficial velocities, the spatially averaged MAV at $0.16 \mathrm{~m} / \mathrm{s}$ was $12.5 \%$ greater in comparison to $0.12 \mathrm{~m} / \mathrm{s}$; this result was expected due to the prevailing liquid MAVs at their respective conditions. Furthermore, as expected, it can be seen in Figure 28c the PVF decreased with increasing liquid superficial velocity. The increase in the PVF for 0.16 and 0.20 $\mathrm{m} / \mathrm{s}$ close to wall can be described by the circulation pattern previously explained (the particle 
moved downwards close the wall). At $0.20 \mathrm{~m} / \mathrm{s}$, the PVF showed two minimum regions off-center, and for $0.16 \mathrm{~m} / \mathrm{s}$, a single minimum near the center. The observations were due to the gulfstreaming effect, where the continuous displacement of high velocity particles is expected to slightly decrease the concentration of particles in that region. The PVF trend for $0.12 \mathrm{~m} / \mathrm{s}$ showed an irregular trend with various minor peaks, with the highest PVF near the center of the bed. This may also be attributed to the very low stability of the bed.

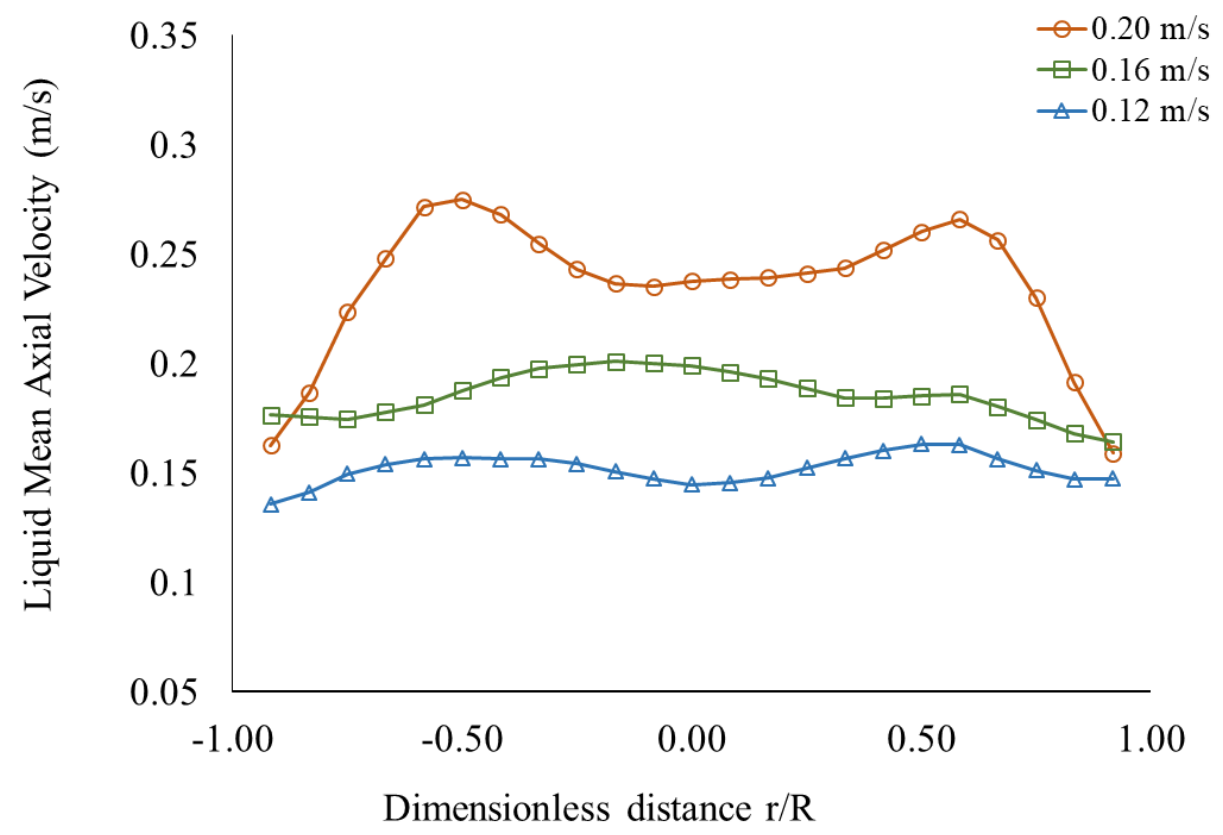

(a) 


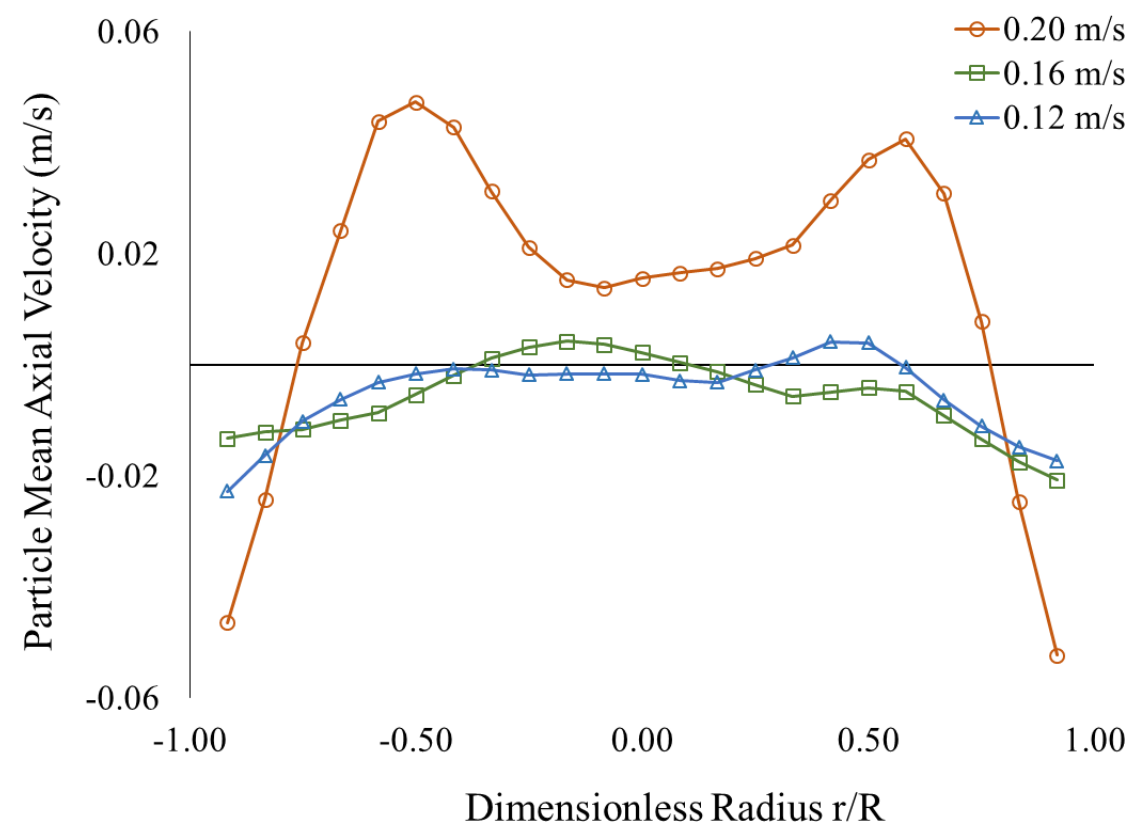

(b)

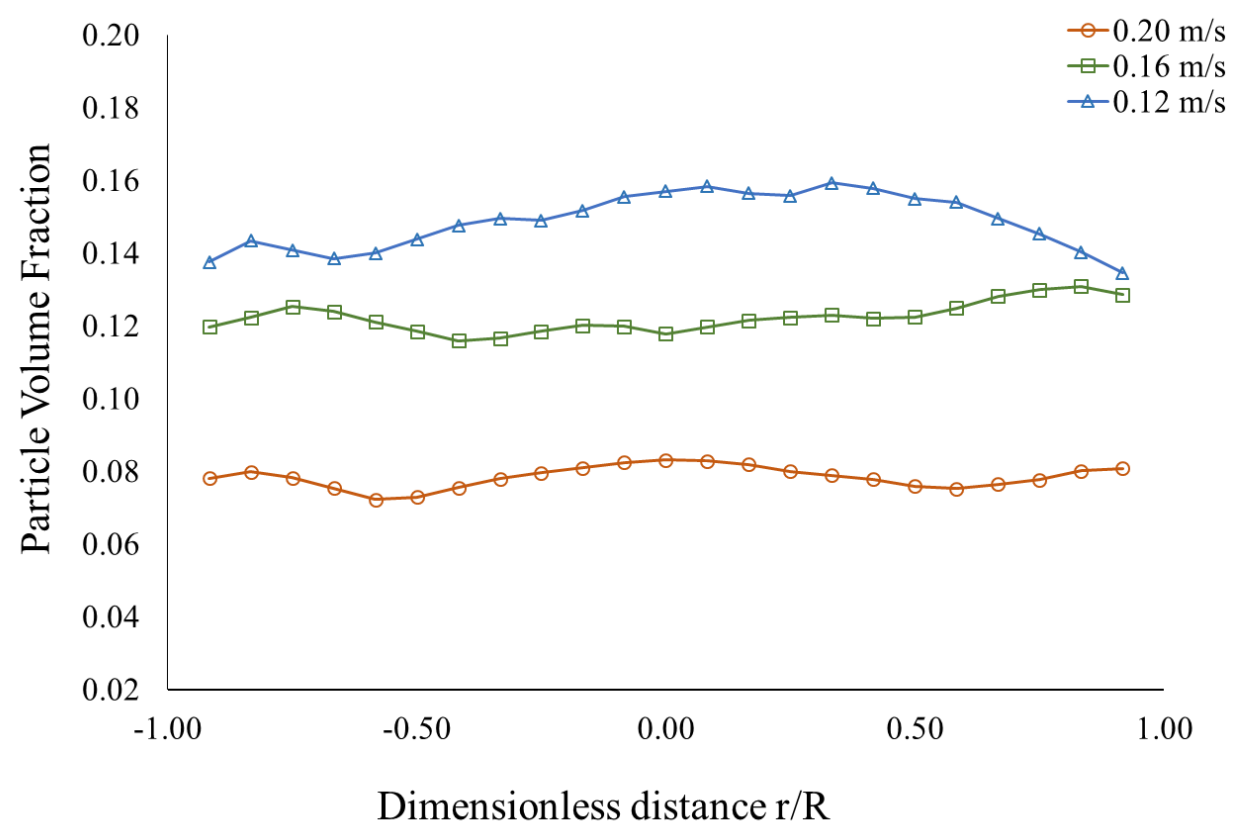

(c)

Figure 28: (a) Liquid MAV, (b) particle MAV and (c) PVF along the radial direction for 50,000 particles fluidized at various liquid superficial velocities calculated at $0.12 \mathrm{~m}$ bed height.

Results in this study showed that the LSFB hydrodynamics were completely altered with the change in liquid superficial velocity. Furthermore, bed stability at the prevailing liquid superficial velocity played a significant role in defining the symmetricity of the particle and liquid flow behavior. 


\subsection{The Effect of Number of Particles on System Hydrodynamics}

The effect of varying the number of particles on the hydrodynamic behavior within a LSFB was investigated by fluidizing 50,000, 75,000, and 100,000 particles at $0.16 \mathrm{~m} / \mathrm{s}$ liquid superficial velocity. The selected range of number of particles was due to computational time restrictions. This section addresses the bed stability and liquid and particle flow behavior at the aforementioned conditions.

\subsubsection{Stability Analysis for Various Number of Particles}

Figure 29 shows the Wallis (1962) stability criterion for $0.002 \mathrm{~m}$ glass beads fluidized with water as a function of the bed porosity. The colored data points in Figure 29 represent the bed-averaged porosity calculated from the CFD-DEM results (averaged over 10 seconds of simulation) for the various number of particles studied. As can be seen, the bed stability increased with decreasing number of particles (for the range investigated).

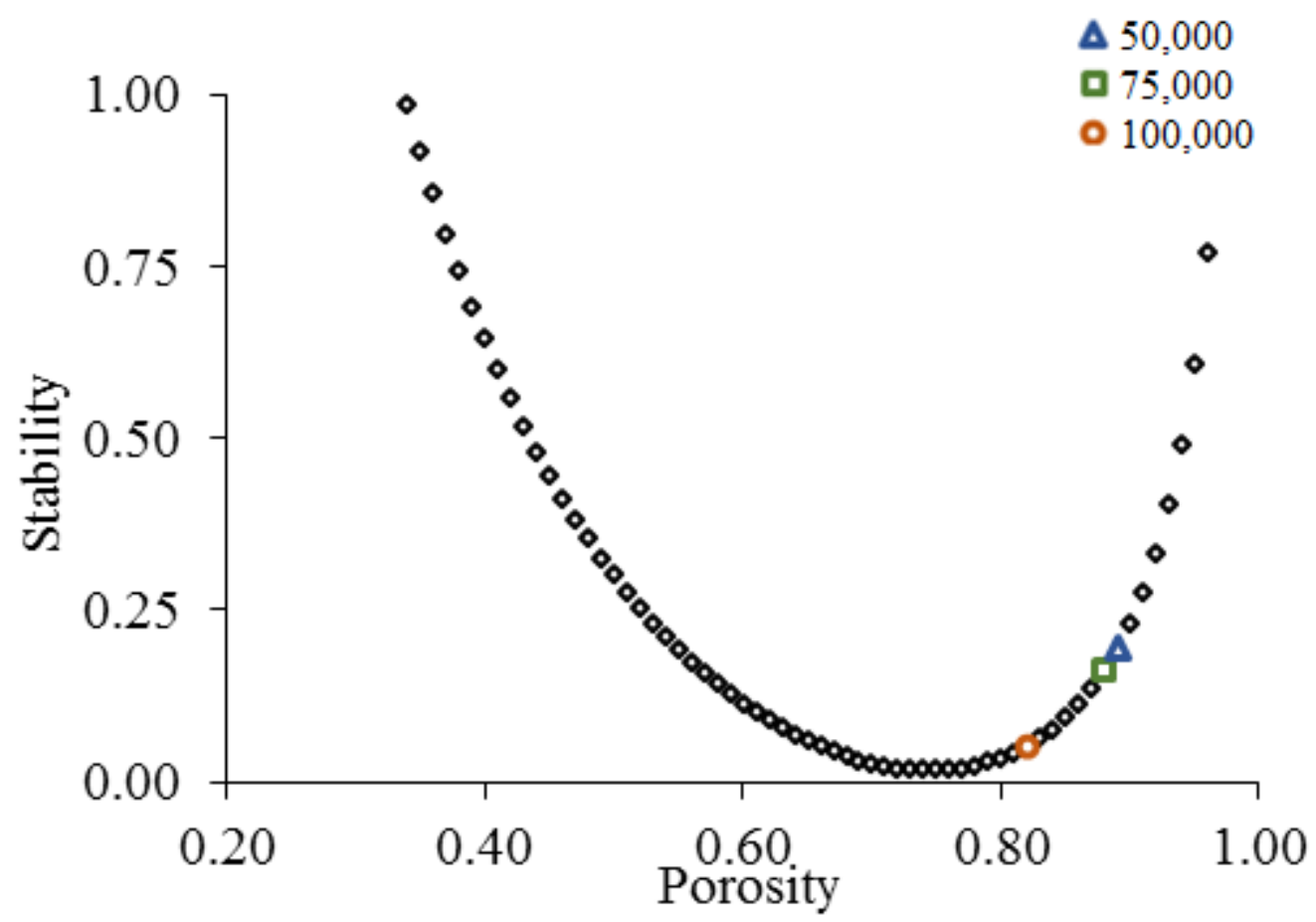

Figure 29: Stability as a function of porosity for $0.002 \mathrm{~m}$ glass beads fluidized with water. Porosities calculated from the CFD-DEM results at various number of particles are indicated by colored points.

In this study, it was found that the transition from one gulf-stream in the center (for 50,000 particles fluidized at $0.16 \mathrm{~m} / \mathrm{s}$ ) to two off-center gulf-streams (similar to 50,000 particles fluidized at 0.20 $\mathrm{m} / \mathrm{s}$ presented in Figure 26) occurred when the number of particles increased from 50,000 to 75,000 
and 100,000. The figures showing gulf-streaming and vortices (as in Figure 26) for 75,000 and 100,000 particles can be seen in the appendix Figures 32 and 33. This proved that the intermediate flow regime was encountered for all number of particles investigated. As presented in section 4.6, an increase in the spatially averaged liquid MAV, due to an increase in liquid superficial velocity from $0.16 \mathrm{~m} / \mathrm{s}$ to $0.20 \mathrm{~m} / \mathrm{s}$, resulted in transition from one gulf-stream to two off-center gulf streams. An increase of number of particles resulted in a decrease in porosity and consequently an increase in the spatially averaged liquid MAV. Therefore, an increase in the number of particles could also lead to the transition from one gulf-stream to two off-center gulf streams.

\subsubsection{The Effect of the Number of Particles on the Liquid and Particle Flow Behavior}

Figure 30 shows the liquid (a) and particle (b) MAVs, and PVF (c) at $0.12 \mathrm{~m}$ for simulations including various number of particles fluidized at $0.16 \mathrm{~m} / \mathrm{s}$ liquid superficial velocity. As can be seen in Figure 30a, the LSFB including 50,000 particles exhibited the lowest liquid MAV in comparison with simulations including 75,000 and 100,000 particles in the central regions. In general, this result was expected since the 50,000 particles system provided the lowest PVF (i.e. there existed a higher available area for liquid flow, which caused a decrease in the liquid MAV) as seen in Figure 30c. The higher liquid MAV close to the wall for the 50,000 particles LSFB (compared to 75,000 and 100,000 particles systems) was anticipated in order to satisfy the conservation of mass.

Figure 30a also shows two off-center peaks for fluidization of 75,000 and 100,000 particles. However, the former possessed more defined peaks in comparison to the latter, which can be explained by the higher stability encountered for the former vs. the latter (Figure 29). It was also observed that the fluidization of 75,000 particles possessed greater liquid MAV magnitudes at the off-center peaks in comparison to the liquid MAVs of simulations with 100,000 particles. This can also be explained by the prevailing stability at their respective fluidization conditions. For simulations with 75,000 particles (higher stability), the frequency of the appearance and disappearance (alternating effect) of gulf streams was expected to be considerably less in comparison with 100,000 particles (less stable). This implied that the enduring gulf-streams were better established for fluidization of 75,000 particles vs. that of 100,000 particles. However, for simulations with 100,000 particles, a higher frequency of the hydrodynamic shift between vortices 
dominant flow and gulf streaming dominant flow was expected due to the lower stability (See the Appendix Figure 33 for an illustration of gulf-stream and vortex dominated flows). This potentially led to the reduction of the two gulf streams' magnitudes in the off-center for 100,000 particles in comparison with that for 75,000 particles. Although the simulation of 75,000 particles provided greater liquid MAV magnitudes at the off-center peaks in comparison with the simulation of 100,000 particles, the spatially averaged liquid MAV was 5\% greater for the latter vs. former simulations. The lower liquid MAV close to the wall for simulations with 75000 particles (in comparison with 100,000 particles) was expected to satisfy the conservation of mass. Furthermore, as seen for the simulation with 100,000 particles, the liquid velocity profile was slightly asymmetrical and irregular in trend. This can be explained by the near zero stability predicted by the Wallis (1962) criterion at this condition (section 4.7.1).

Figure $30 \mathrm{~b}$ illustrates that the motion of particles followed that of the liquid. Moreover, this figure shows the particle circulation pattern, downwards near the wall and upwards throughout the rest of the bed, existed for all particle numbers investigated. For the fluidization of 50,000 particles, a maximum in particle MAVs was observed very close to the center. This was a direct result of the single gulf-stream which was encountered at this condition, as previously discussed in section 4.6.2. For the fluidization of 75,000 particles and 100,000 particles, a maximum in particle MAVs was observed at the two off-center locations. This was a direct result of the two gulf-streams previously discussed for these conditions. This result implied that the particle circulation was greatly more pronounced in the off-center in comparison to the near center location of the bed. For the fluidization of 75,000 particles, the higher liquid MAV peaks at the off-center, and the lower liquid velocity close to wall allowed particles in this case to move downwards with higher velocity compared to the other cases including 50,000 and 100,000 particles. Although the fluidization of 75,000 particles possessed greater particle MAV magnitudes at the off-center peaks in comparison to simulations with 100,000 particles, the spatially averaged particle MAVs were $63 \%$ greater for the latter compared to the former system.

Figure 30c shows that on average the PVF increased with increasing number of particles, as expected. Furthermore, the symmetricity in the PVF trends appeared to increase with decreasing number of particles, and increasing bed stability. 


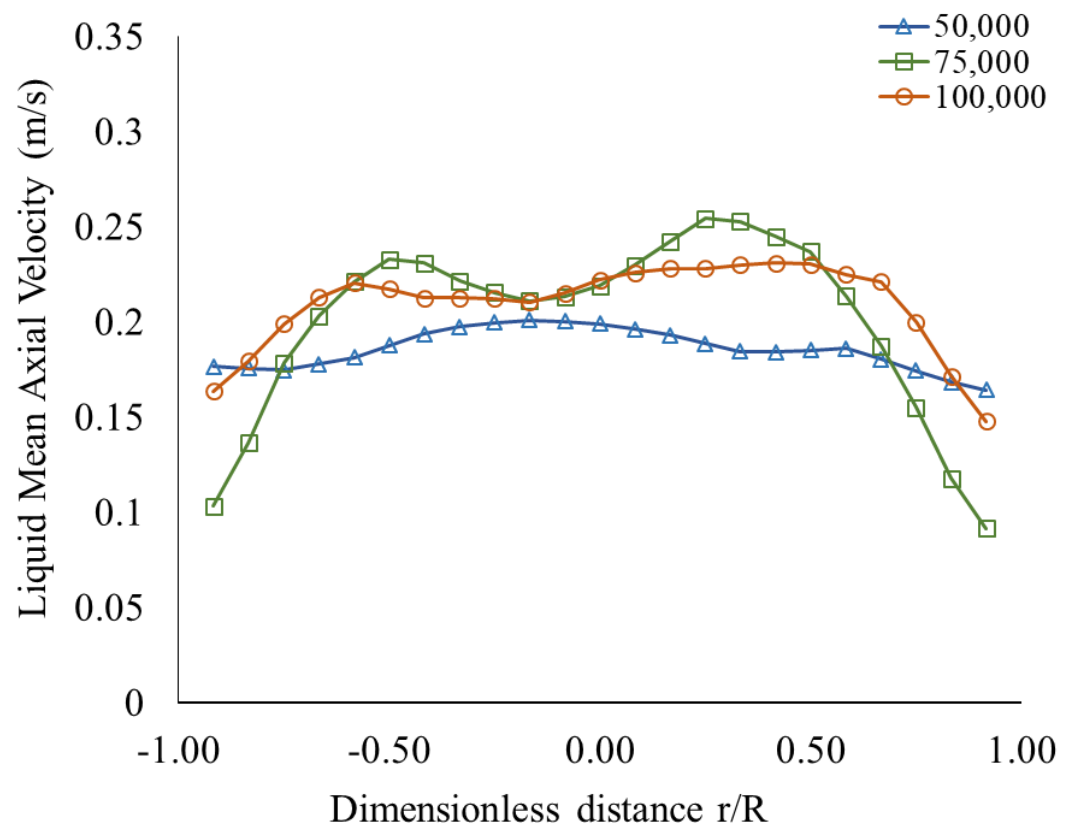

(a)

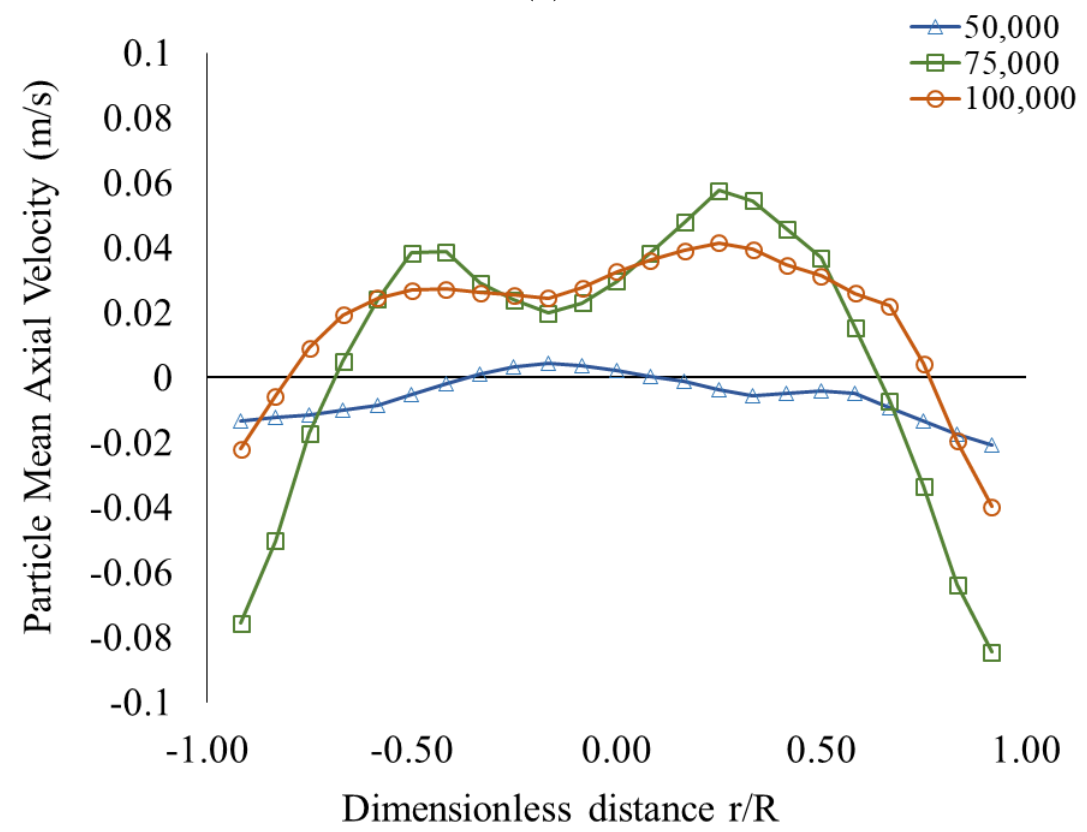

(b) 


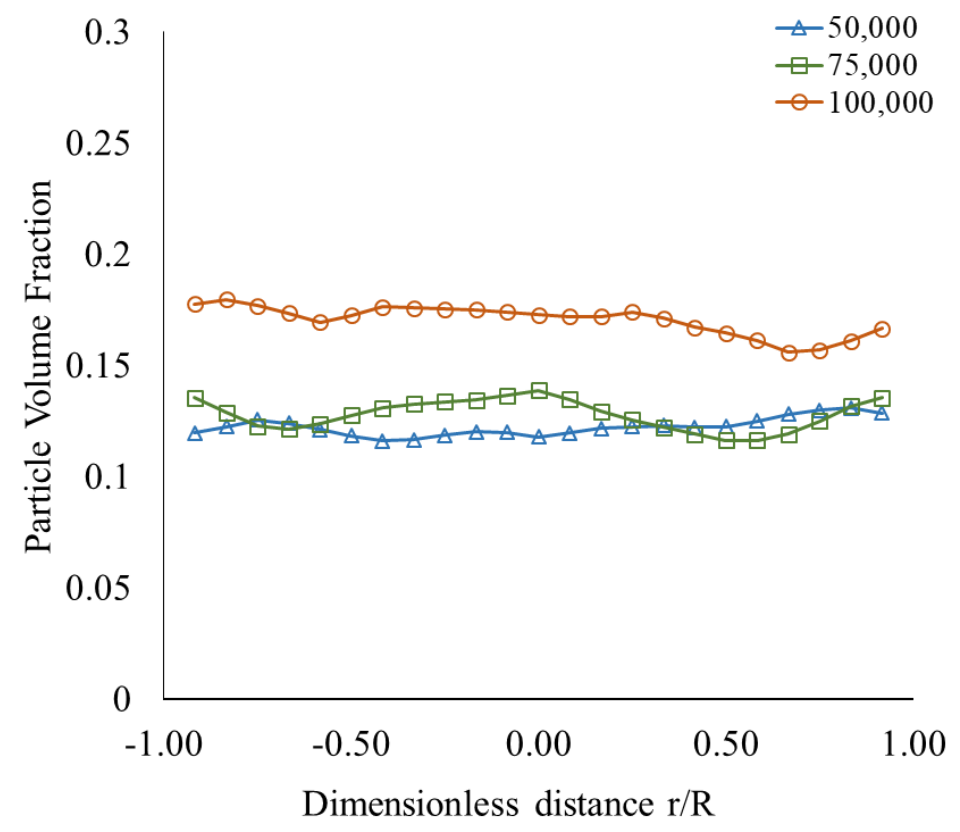

(c)

Figure 30: (a) Liquid MAV, (b) particle MAV and (c) PVF along the radial direction for various number of particles fluidized at $0.16 \mathrm{~m} / \mathrm{s}$ liquid superficial velocity calculated at $0.12 \mathrm{~m}$.

In literature of CFD-DEM simulations of LSFBs, a few studies reduced the number of particles compared to experiments to decrease the computational time (Di Renzo et al., 2011; Peng et al., 2016). Although the geometry was scaled down to account for the lesser number of particles, the scale down procedure was not presented by these studies. The scale down of the experimental setup can be particularly problematic when hydrodynamic similarity is not guaranteed. As was shown in this study, LSFBs behave differently when different particle weight fractions are chosen, and close attention must be paid when attempting to reduce the number of particles employed to approximate experiments with higher number of particles. 


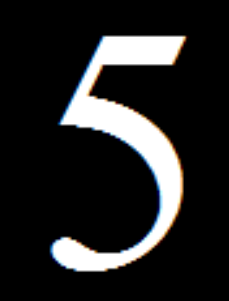

Conclusions \&

Recommendations 
In this study, tomography experiments, statistical modeling, CFD-DEM simulations, and stability analysis were applied to thoroughly investigate a liquid-solid fluidized bed system. ERT was employed to measure the particle concentration at various bed heights in the LSFB for various particle numbers and liquid flow rates. The experimental results were then used to develop a statistical model which could be employed as a tool to predict the BPVF within the studied range of the design variables.

The CFD-DEM model was validated using the ERT experimental method. Initially, it was desired to quantify the influence of each interaction force (drag, pressure gradient, virtual mass, and Saffman lift forces) on simulation results. Since the drag force is the most dominant interaction force, three common drag models were compared: The Naumann-Schiller, Gidaspow, and Syamlal-O’brien drag models. Simulations employing the Gidaspow drag model were found to be in closest agreement with experimental results.

Pursuant to drag model analysis, the effect of the inclusion of individual interaction forces (other than drag) was investigated. It was found that the inclusion of pressure gradient and virtual mass forces, alongside Gidaspow drag, resulted in a reduction in percentage error (i.e. 10.30\%). However, the addition of Saffman lift, alongside Gidaspow drag, virtual mass, and pressure gradient forces, caused a considerable overestimation of the experimental results. Each interaction force was quantified, and the drag force was observed to be considerably greater in magnitude in comparison to other interaction forces. This signified the drag force's dominance, in terms of its influence on the macroscopic behavior of LSFBs, in comparison to other interaction forces.

Contact parameters calibration was conducted to further analyze their effect on the CFD-DEM simulations. The COR, $\mu_{\mathrm{s}}$, and $\mu_{\mathrm{r}}$ were adjusted for P-P and P-W contacts. It was generally observed that dry particle contact parameters should not be employed in wet particle systems. It was concluded that a lower than 'dry' COR for particle-particle and particle-wall contacts needed to be employed in LSFB CFD-DEM simulations to capture the viscous dissipation effect. It was also concluded that employing $0.3 \mu_{\mathrm{s}} \mathrm{P}-\mathrm{W}$ and $0.1 \mu_{\mathrm{s}} \mathrm{P}-\mathrm{P}$ values resulted in closest agreement with experimental results, as these values accounted for lubrication effects due to the presence of an interstitial fluid between particles and particle-wall. The difference between $\mu_{\mathrm{s}} \mathrm{P}-\mathrm{P}$ and $\mu_{\mathrm{s}} \mathrm{P}-\mathrm{W}$ was attributed to the difference between particle and geometry materials. Calibration analysis of $\mu_{\mathrm{r}}$ demonstrated that employing a small value of $\mu_{\mathrm{r}}(0.01)$ could not account for particle rotation 
damping occurring due to the presence of a liquid in a LSFB. Alternatively, choosing a high value for $\mu_{\mathrm{r}}(0.3)$ overestimated the particle rotation damping, leading to an increase in percentage error. A value of $0.1 \mu_{\mathrm{r}}$, for both P-W and P-P, was found to provide the closest prediction of experimental data.

Furthermore, stability analysis and CFD-DEM simulations were applied to investigate a LSFB operating in the intermediate flow regime. The simulation results were employed to obtain particle PVFs, liquid and particle MAVs, and porosity values at various liquid superficial velocities and number of particles. The liquid superficial velocity in this study was varied from $0.12 \mathrm{~m} / \mathrm{s}$ to 0.16 $\mathrm{m} / \mathrm{s}$ and $0.20 \mathrm{~m} / \mathrm{s}$ while maintaining the number of particle constant at 50,000. Also, the number of particles investigated in this study was 50,000, 75,000, and 100,000, while maintaining the liquid superficial velocity at $0.16 \mathrm{~m} / \mathrm{s}$. Based on the Wallis stability criterion and porosity values obtained from the CFD-DEM simulations, it was found that the intermediate flow regime was encountered for all cases studied in this research. Furthermore, the Wallis criterion predicted an increase in stability with an increase in liquid superficial velocity and a decrease in the number of particles (for the range studied).

For simulations with varying liquid superficial velocities (50,000 particles), the time averaged PVF along the bed height was extracted from the CFD-DEM results. For $0.12 \mathrm{~m} / \mathrm{s}$ (lowest stability case), it was found that the PVF trend exhibited a highly irregular behavior. This confirmed the intermediate flow regime for $0.12 \mathrm{~m} / \mathrm{s}$ fluidization since a constant PVF trend is expected for homogeneous flow regimes. An increase in liquid superficial velocity to $0.16 \mathrm{~m} / \mathrm{s}$ resulted in decrease in the irregularity in the PVF trend, implying an increase in stability as predicted by the Wallis criterion. The intermediate flow regime was confirmed due to the decreasing PVF trend observed at $0.16 \mathrm{~m} / \mathrm{s}$. Further increase in the liquid superficial velocity to $0.20 \mathrm{~m} / \mathrm{s}$ resulted in the highest stability case where the PVF trend was inconclusive in determining the prevailing flow regime. For $0.20 \mathrm{~m} / \mathrm{s}$, the intermediate flow regime was demonstrated by presenting the appearance and disappearance of the gulf-streaming effect which is indicative of non-homogeneity; in the absence of the bed bubbling phenomenon. It was further shown that a transition from a single central gulf-stream to two off-center gulf streams occurred when the liquid superficial velocity was increased from $0 / 16 \mathrm{~m} / \mathrm{s}$ to $0.20 \mathrm{~m} / \mathrm{s}$. 
For simulations with varying number of particles (at $0.16 \mathrm{~m} / \mathrm{s}$ liquid superficial velocity), the intermediate flow regime was confirmed by observing the liquid and particle MAVs and noting the gulf-streaming effect in each case. It was found that a transition from a single gulf-stream to two off-center gulf streams could also occur when increasing the number of particles from 50,000 to 75,000 and 100,000 . It was concluded that an increase in the number of particles caused a decrease in porosity which ultimately resulted in an increase in liquid and particle MAVs; this may have caused the transition.

It was shown that in general, the particle moved upwards in the central areas of the LSFBs and downwards closer to the wall creating a particle circulation pattern; with the exception of $0.12 \mathrm{~m} / \mathrm{s}$ fluidization of 50,000 particles where an irregular circulation pattern was observed (due to very low stability and highly dense system). It was further noticed that due to the different location of the gulf-streaming effect (single gulf-stream in the center of the bed vs. two off-center gulfstreams), a completely different particle circulation pattern existed at different fluidization conditions.

In this study, the link between particle-scale phenomena and the macroscopic behavior of LSFBs was demonstrated. It was concluded that close attention must be paid before a specific drag model is selected, individual interaction forces are omitted, and particle contact parameters are chosen. Furthermore, results in this study revealed that the hydrodynamic behavior of LSFBs operating in the intermediate flow regime is highly dependent on bed stability, which is ultimately a function of both the number of particles and liquid superficial velocity.

In future studies it is recommended:

- To investigate the effect of particle size change on the hydrodynamic behavior of LSFBs.

- To conduct CFD-DEM simulations of LSFBs that include a particle size distribution.

- To perform CFD-DEM simulations of LSFBs with non-spherical particles as this is more applicable to industrial processes.

- To explore the scale-up of the studied system and to observe whether hydrodynamic similarity is achievable.

- To conduct CFD-DEM simulations of LSFBs with a non-Newtonian fluid. 
Conclusions and Recommendations

- To develop the CFD-DEM model to include heat and mass transfer, thus increasing the model's applicability to most industrial processes. 
Appendix 
file $6=0$; file $12=0$; file $18=0$; file $24=0$; \%initialize all values $\mathrm{y} 6=0 ; \mathrm{y} 12=0 ; \mathrm{y} 18=0 ; \mathrm{y} 24=0 ; \%$ initialize all values $\mathrm{x} 6=0 ; \mathrm{x} 12=0 ; \mathrm{x} 18=0 ; \mathrm{x} 24=0 ; \%$ initialize all values $\mathrm{m} 6=0 ; \mathrm{m} 12=0 ; \mathrm{m} 18=0 ; \mathrm{m} 24=0 ; \%$ initialize all values $\mathrm{z6}=0 ; \mathrm{z} 12=0 ; \mathrm{z} 18=0 ; \mathrm{z} 24=0 ; \%$ initialize all values num $=0 ; \%$ number of files averaged initialization for ii $=5000: 50: 10000 \%$ average only from 10 s to 20 s; every $0.1 \mathrm{~s}$

file6 = ['plane-at-6cm-',num2str(ii)]; \%read file at $6 \mathrm{~cm}$ y6 = dlmread(file6, ',',1,4); \%start reading the delimited file6, 1st row 4th column (concentration values)

$\mathrm{x} 6=\mathrm{y} 6(1: 263) ; \%$ store all values in the 4th column from row 1 to 263 in $x 6$ $\mathrm{m} 6=$ mean $(\mathrm{x} 6) ; \%$ find the average of the values in $\mathrm{x} 6$

$\mathrm{z} 6=\mathrm{z} 6+\mathrm{m} 6 ; \%$ store the $\mathrm{x} 6$ value in $\mathrm{z} 6$ so that all iteration averages can be obtained

file12 = ['plane-at-12cm-',num2str(ii)]; \%read file at $12 \mathrm{~cm}$ y12 = dlmread(file12, ',',1,4); \%start reading the delimited file12, 1st row 4th column (concentration values)

$\mathrm{x} 12=\mathrm{y} 12(1: 263) ; \%$ store all values in the 4 th column from row 1 to 263 in $\mathrm{x} 12$ $\mathrm{m} 12=$ mean $(\mathrm{x} 12) ; \%$ find the average of the values in $\mathrm{x} 12$

$\mathrm{z} 12=\mathrm{z} 12+\mathrm{m} 12 ; \%$ store the $\mathrm{x} 12$ value in $\mathrm{z} 12$ so that all iteration averages can be obtained

file18 = ['plane-at-18cm-',num2str(ii)]; \%read file at $18 \mathrm{~cm}$ y18 = dlmread(file18, ',',1,4); \%start reading the delimited file18, 1st row 4th column

$\mathrm{x} 18=\mathrm{y} 18(1: 263) ; \%$ store all values in the 4 th column from row 1 to 263 in $\mathrm{x} 18$ $\mathrm{m} 18=$ mean $(\mathrm{x} 18) ; \%$ find the average of the values in $\mathrm{x} 18$

$\mathrm{z} 18=\mathrm{z} 24+\mathrm{m} 18 ; \%$ store the $\mathrm{x} 18$ value in $\mathrm{z} 18$ so that all iteration averages can be obtained

file24 = ['plane-at-24cm-',num2str(ii)]; \%read file at $24 \mathrm{~cm}$ y24 = dlmread(file24, ',',1,4); \%start reading the delimited file24, 1st row 4th column

$\mathrm{x} 24=\mathrm{y} 24(1: 263) ; \%$ store all values in the 4th column from row 1 to 263 in $\mathrm{x} 24$ $\mathrm{m} 24=$ mean $(\mathrm{x} 24) ; \%$ find the average of the values in $\mathrm{x} 24$

$\mathrm{z} 24=\mathrm{z} 24+\mathrm{m} 24 ; \%$ store the $\mathrm{x} 24$ value in $\mathrm{z} 24$ so that all iteration averages can be obtained

num $=$ num $+1 ; \%$ total number of files averaged end

$\mathrm{c} 6=\mathrm{z} 6 / \mathrm{num} \%$ find the average concentration at $6 \mathrm{~cm}$ $\mathrm{c} 12=\mathrm{z} 12 /$ num \% find the average concentration at $12 \mathrm{~cm}$ $\mathrm{c} 18=\mathrm{z} 18 /$ num \%find the average concentration at $18 \mathrm{~cm}$ c24 $=\mathrm{z} 24 /$ num \%find the average concentration at $24 \mathrm{~cm}$

Figure 31: MATLAB code to obtain simulation PPVF. 
Appendix
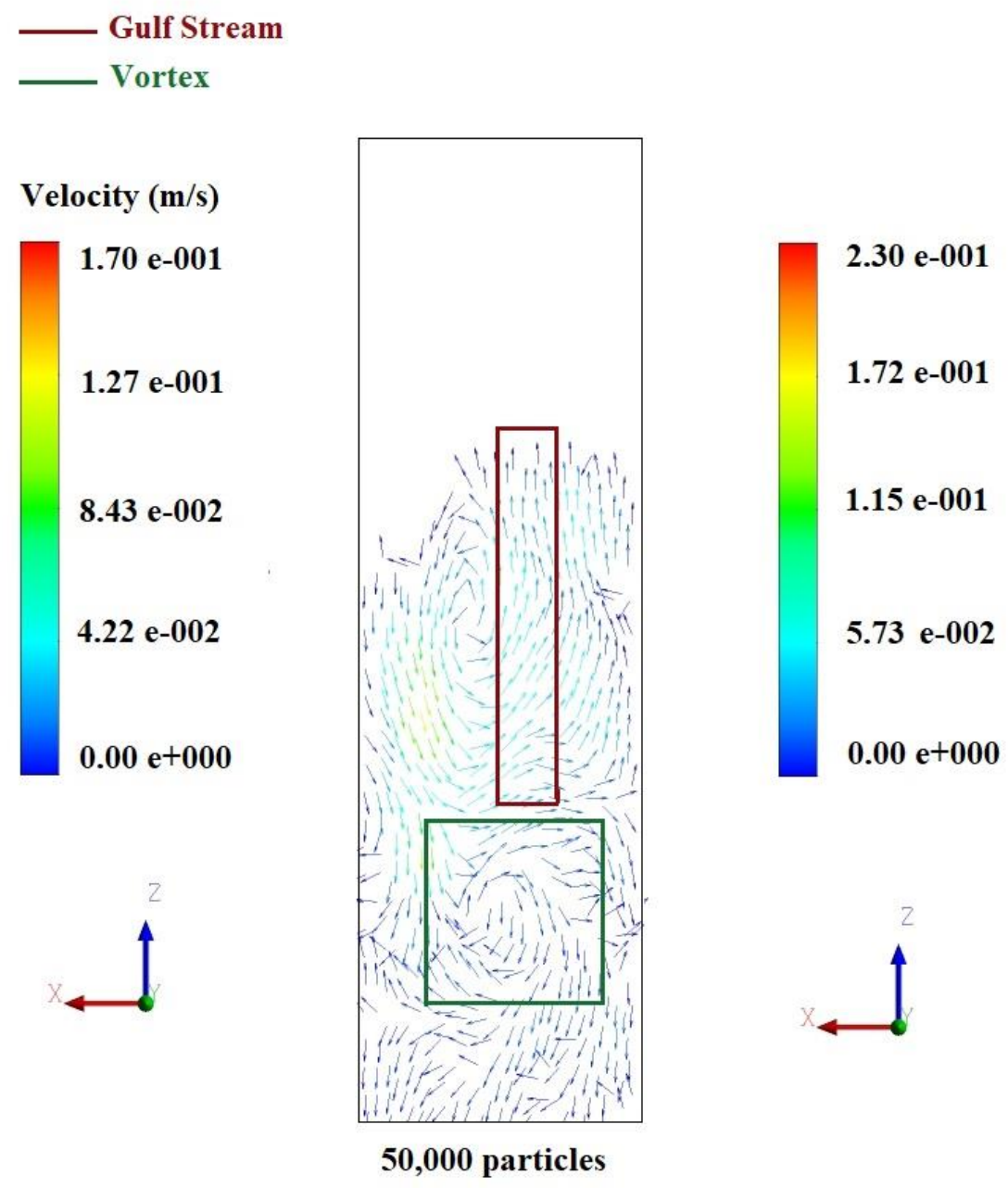

Figure 32: Gulf Streaming and Vortices for 50,000 and 75,000 particles fluidized at $0.16 \mathrm{~m} / \mathrm{s}$. 
Appendix

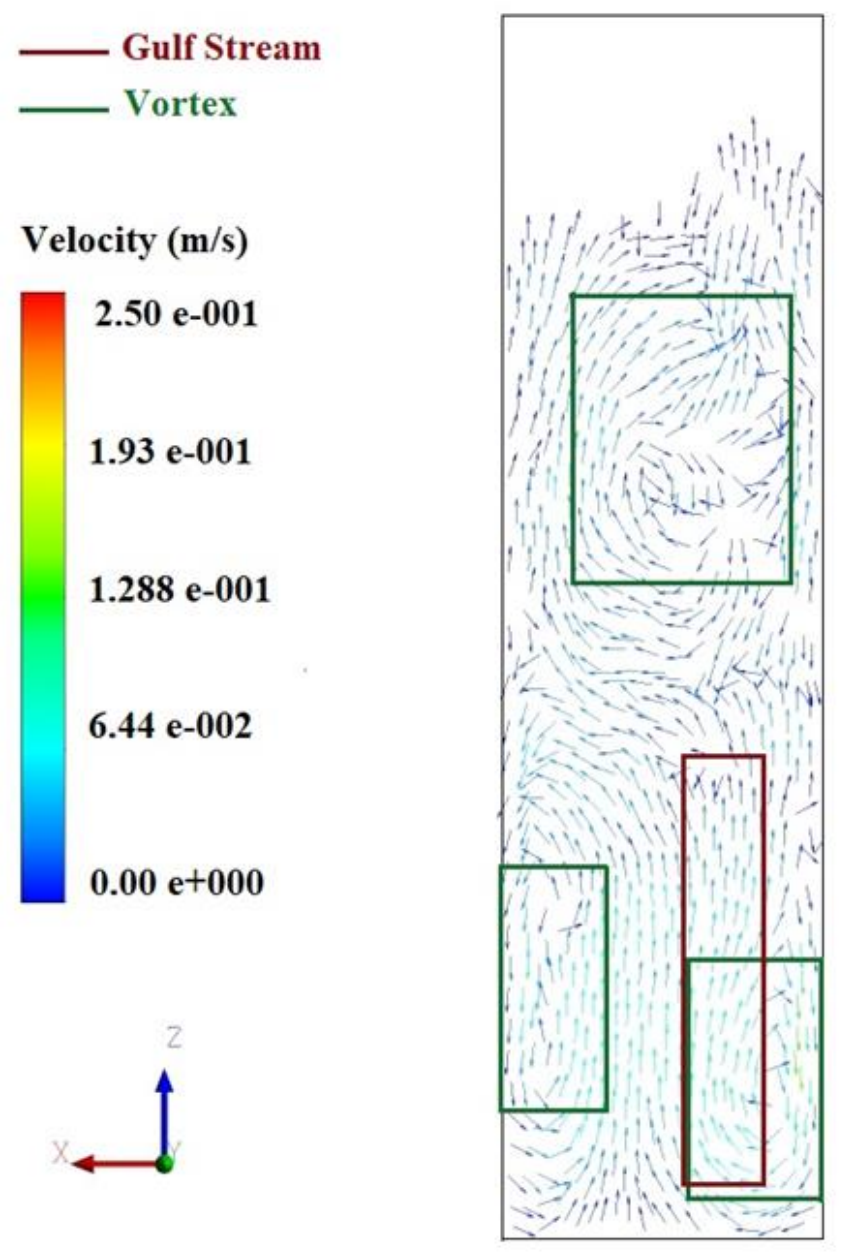

Vortex dominated Flow

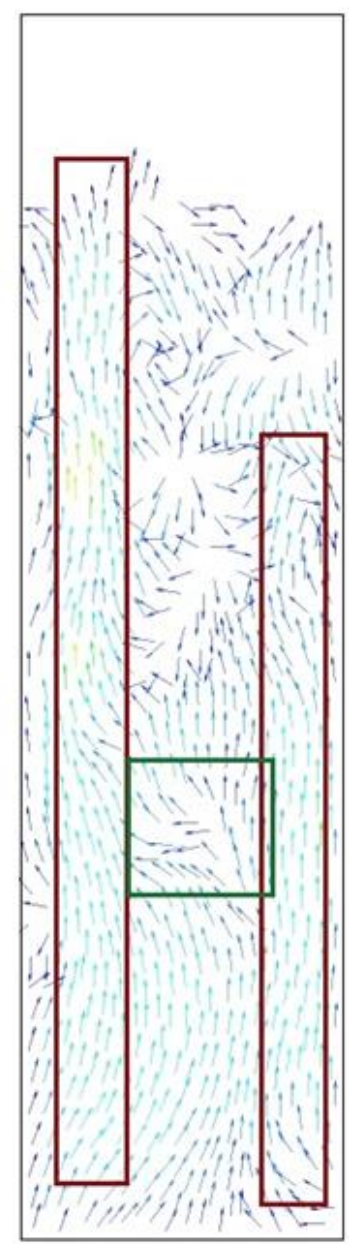

Gulf Stream dominated Flow

$18 \mathrm{~s}$

Figure 33: Gulf Streaming and Vortex dominated flows for 100,000 particles fluidized at $0.16 \mathrm{~m} / \mathrm{s}$. 


\section{7}

References 
Ai, J., Chen, J. F., Rotter, J. M., \& Ooi, J. Y. (2011). Assessment of rolling resistance models in discrete element simulations. Powder Technology, 206(3), 269-282. https://doi.org/10.1016/j.powtec.2010.09.030

Al-Arkawazi, S., Marie, C., Benhabib, K., \& Coorevits, P. (2017). Modeling the hydrodynamic forces between fluid-granular medium by coupling DEM-CFD. Chemical Engineering Research and Design, 117, 439-447. https://doi.org/10.1016/j.cherd.2016.11.002

Anderson, J. D. J., \& Wendt, J. (1995). Computational Fluid Dynamics. Retrieved from http://www.ulb.tu-darmstadt.de/tocs/207110735.pdf

Anderson, T. B., \& Jackson, R. (1967). Fluid mechanical description of fluidized beds: Equations of Motion. Industrial and Engineering Chemistry Fundamentals, 6(4), 527-539. https://doi.org/10.1021/i160024a007

Aw, S. R., Rahim, R. A., Rahiman, M. H. F., Yunus, F. R. M., \& Goh, C. L. (2014). Electrical resistance tomography: A review of the application of conducting vessel walls. Powder Technology, 254, 256-264. https://doi.org/10.1016/j.powtec.2014.01.050

Barber, C. C., Brown, B. H., \& Freeston, I. L. (1983). Imaging spatial distributions of resistivity using applied potential tomography. Electronics Letters, 19(22), 933 -935. https://doi.org/10.1049/el:19830637

Barber, D. C., Oubel, E., Frangi, A. F., \& Hose, D. R. (2007). Efficient computational fluid dynamics mesh generation by image registration. Medical Image Analysis, 11(6), 648-662. https://doi.org/10.1016/j.media.2007.06.011

Beetstra, R., Van der Hoef, M. A., \& Kuipers, J. A. M. (2007). Drag force of intermediate reynolds number flow past mono- and bidisperse arrays of spheres. AIChE Journal, 53(2), 489-501. https://doi.org/10.1002/aic.11065

Bird, R. B., Stewart, W. E., \& Lightfoot, E. N. (2002). Transport Phenomena. John Wiley and Sons, Inc. New York. https://doi.org/10.1016/j.ijhydene.2006.08.059

Blais, B., \& Bertrand, F. (2015). On the use of the method of manufactured solutions for the verification of CFD codes for the volume-averaged Navier-Stokes equations. Computers and Fluids, 114, 121-129. https://doi.org/10.1016/j.compfluid.2015.03.002 
Blais, B., \& Bertrand, F. (2017). CFD-DEM investigation of viscous solid-liquid mixing: Impact of particle properties and mixer characteristics. Chemical Engineering Research and Design, 118, 270-285. https://doi.org/10.1016/j.cherd.2016.12.018

Box, G. E. P., \& Behnken, D. W. (1960). Some New Three Level Designs for the Study of Quantitative Variables. $\quad$ Technometrics, $\quad$ 2(4), 455-475. https://doi.org/10.1080/00401706.1960.10489912

Box, G. E. P., \& Wilson, K. B. (1951). On the experimental attainment of optimum conditions. Journal of the Royal Statistical Society, 13(1), 1-45. https://doi.org/10.1007/978-1-4612-43809_23

Buck, B., Tang, Y., Heinrich, S., Deen, N. G., \& Kuipers, J. A. M. (2017). Collision dynamics of wet solids: Rebound and rotation. Powder Technology, 316, 218-224. https://doi.org/10.1016/j.powtec.2016.12.088

Cello, F., Di Renzo, A., \& Di Maio, F. P. (2009). DEM-CFD simulations of layer inversion in twocomponent liquid fluidized beds. Chemical Engineering Transactions, 17, 627-632. https://doi.org/10.3303/CET0917105

Cello, F., Di Renzo, A., \& Di Maio, F. P. (2010). A semi-empirical model for the drag force and fluid-particle interaction in polydisperse suspensions. Chemical Engineering Science, 65(10), 3128-3139. https://doi.org/10.1016/j.ces.2010.02.006

Çengel, Y. A., \& Cimbala, J. M. (2006). Fluid mechanics: fundamentals and applications. Fluid Mechanics: With Problems and Solutions, and an Aerodynamic Laboratory, 956. https://doi.org/10.1088/1751-8113/44/8/085201

Chaouki, J., Larachi, F., \& Duduković, M. P. (1997). Noninvasive Tomographic and Velocimetric Monitoring of Multiphase Flows. Industrial and Engineering Chemistry Research, 36(11), 44764503. https://doi.org/10.1021/ie970210t

Chung, T. J. (2009). Computational Fluid Dynamics. The British journal of radiology (Vol. 82). https://doi.org/10.1007/s10915-006-9104-X 
Cook, B. K., Noble, D. R., \& Williams, J. R. (2004). A direct simulation method for particle-fluid systems. $\quad$ Engineering Computations, 21(2/3/4), 151-168. https://doi.org/10.1108/02644400410519721

Couderc, J.P. (1985). Incipient Fluidization and Particulate Systems. Fluidization (2 ${ }^{\text {nd }}$ ed.). Academic Press. New York. (pp. 1-44).

Cundall, P. A., \& Strack, O. D. L. (1979). A discrete numerical model for granular assemblies. Géotechnique, 29(1), 47-65. https://doi.org/10.1680/geot.1979.29.1.47

Dallavalle, J. (1948). Micromeritics (Vol. 56). Pitman Publishing Corporation. New York.

David M. Scott, H. M. (2005). Process Imaging for Automatic Control. CRC Press: Taylor \& Francis Group. Boca Raton. https://doi.org/10.1017/CBO9781107415324.004

De Santis, G., Mortier, P., De Beule, M., Segers, P., Verdonck, P., \& Verhegghe, B. (2010). Patient-specific Computational Fluid Dynamics: Structured mesh generation from coronary angiography. Medical and Biological Engineering and Computing, 48(4), 371-380. https://doi.org/10.1007/s11517-010-0583-4

Deen, N. G., Van Sint Annaland, M., Van der Hoef, M. A., \& Kuipers, J. A. M. (2007). Review of discrete particle modeling of fluidized beds. Chemical Engineering Science, 62(1-2), 28-44. https://doi.org/10.1016/j.ces.2006.08.014

DEMSolutions. (2010). EDEM-CFD coupling for FLUENT: User Guide. Edinburgh.

Di Felice, R. (1994). The voidage function for fluid-particle interaction systems. International Journal of Multiphase Flow, 20(1), 153-159. https://doi.org/10.1016/0301-9322(94)90011-6

Di Felice, R. (1995). Hydrodynamics of liquid fluidisation. Chemical Engineering Science, 50(8), 1213-1245. https://doi.org/10.1016/0009-2509(95)98838-6

Di Maio, F. P., Di Renzo, A., \& Cello, F. (2008). A new drag force model for detailed simulations of fluid-particle systems. In 11th International Conference on Multiphase Flow in Industrial Plant (pp. 243-250). Palermo, Italy. 
Di Renzo, A., Cello, F., \& Di Maio, F. P. (2011). Simulation of the layer inversion phenomenon in binary liquid--fluidized beds by DEM-CFD with a drag law for polydisperse systems. Chemical Engineering Science, 66(13), 2945-2958. https://doi.org/10.1016/j.ces.2011.03.035

Di Renzo, A., \& Di Maio, F. P. (2004). Comparison of contact-force models for the simulation of collisions in DEM-based granular flow codes. Chemical Engineering Science, 59(3), 525-541. https://doi.org/10.1016/j.ces.2003.09.037

Di Renzo, A., \& Di Maio, F. P. (2007). Homogeneous and bubbling fluidization regimes in DEMCFD simulations: Hydrodynamic stability of gas and liquid fluidized beds. Chemical Engineering Science, 62(1-2), 116-130. https://doi.org/10.1016/j.ces.2006.08.009

Dong, F., Xu, C., Zhang, Z., \& Ren, S. (2012). Design of parallel electrical resistance tomography system for measuring multiphase flow. Chinese Journal of Chemical Engineering, 20(2), 368379. https://doi.org/10.1016/S1004-9541(12)60400-5

Drew, D. A., \& Lahey, R. T. (1990). Some supplemental analysis concerning the virtual mass and lift force on a sphere in a rotating and straining flow. International Journal of Multiphase Flow, 16(6), 1127-1130. https://doi.org/10.1016/0301-9322(90)90110-5

Ebrahimi, M. (2014). CFD-DEM modelling of two-phase pneumatic conveying with experimental validation. The University of Edinburg. Edinburg.

Elghobashi, S. (1991). Particle-laden turbulent flows: direct simulation and closure models. Applied Scientific Research, 48(3-4), 301-314. https://doi.org/10.1007/BF02008202

Epstein, N. (2002). Applications of Liquid-Solid Fluidization. International Journal of Chemical Reactor Engineering, 1(1). https://doi.org/10.2202/1542-6580.1010

Epstein, N. (2003). Liquid-Solids Fluidization. Handbook of Fluidization and Fluid-Particle Systems. Marcel Dekker, Inc. New York. https://doi.org/10.1016/S1672-2515(07)60126-2

Ergun, S. (1952). Fluid flow through packed columns. Chemical Engineering Progress, 48, 8994. https://doi.org/10.1029/JB088iS01p0B353 
Esteghamatian, A., Hammouti, A., Lance, M., Wachs, A., Esteghamatian, A., Hammouti, A., Wachs, A. (2017). Particle resolved simulations of liquid/solid and gas/solid fluidized beds. Physics of Fluids, 29. https://doi.org/10.1063/1.4979137

Fan, L. -S, Matsuura, A., \& Chern, S. H. (1985). Hydrodynamic characteristics of a gas-liquidsolid fluidized bed containing a binary mixture of particles. AIChE Journal, 31(11), 1801-1810. https://doi.org/10.1002/aic.690311106

Foscolo, P., \& Gibilaro, L. (1987). Fluid-dynamic stability of fluidized suspensions: the particle bed model. Chemical Engineering Science, 42(6), 1489-1500

Ge, J., Zhang, D., Tian, W., Wang, K., Qiu, S., \& Su, G. H. (2015). Steady and transient solutions of neutronics problems based on finite volume method (FVM) with a CFD code. Progress in Nuclear Energy, 85, 366-374. https://doi.org/10.1016/j.pnucene.2015.07.012

Ge, W., \& Li, J. (2003). Simulation of particle-fluid systems with macro-scale pseudo-particle modeling. Powder Technology, 137(1-2), 99-108. https://doi.org/10.1016/j.powtec.2003.08.034

Ghafoori, S., Mehrvar, M., \& Chan, P. (2014). Optimisation of photo-Fenton-like degradation of aqueous polyacrylic acid using Box-Behnken experimental design. Canadian Journal of Chemical Engineering, 92(1), 97-108. https://doi.org/10.1002/cjce.21849

Ghatage, S. V., Peng, Z., Sathe, M. J., Doroodchi, E., Padhiyar, N., Moghtaderi, B., Evans, G. M. (2014). Stability analysis in solid-liquid fluidized beds: Experimental and computational. Chemical Engineering Journal, 256, 169-186. https://doi.org/10.1016/j.cej.2014.06.026

Gibilaro, L. G. (2001). Fluidization-dynamics: The formulation and applications of a predictive theory for the fluidized state. Elsevier Ltd. Oxford. https://doi.org/10.1016/B978-075065003$8 / 50015-\mathrm{X}$

Gidaspow, D. (1994). Multiphase flow and fluidization. Journal of Non-Newtonian Fluid Mechanics (Vol. 55). https://doi.org/10.1016/0377-0257(94)80007-3

Gollwitzer, F., Rehberg, I., Kruelle, C. A., \& Huang, K. (2012). Coefficient of restitution for wet particles. Physical Review E - Statistical, Nonlinear, and Soft Matter Physics, 86(1). https://doi.org/10.1103/PhysRevE.86.011303 
Gondret, P., Lance, M., \& Petit, L. (2002). Bouncing motion of spherical particles in fluids. Physics of Fluids, 14(2), 643-652. https://doi.org/10.1063/1.1427920

Myers, R., Montgomery, D., \& Anderson-Cook, M. (2016). Response Surface Methodology: Process and Product Optimization Using Designed Experiments. John Wiley \& Sons. New Jersey. https://doi.org/10.1017/CBO9781107415324.004

Hertz, H. (1882). Ueber die Berührung fester elastischer Körper. Journal Fur Die Reine Und Angewandte Mathematik, 1882(92), 156-171. https://doi.org/10.1515/crll.1882.92.156

Hoomans, B. P. B., Kuipers, J. A. M., \& Van Swaaij, W. P. M. (2000). Granular dynamics simulation of segregation phenomena in bubbling gas-fluidised beds. Powder Technology, 109(13), 41-48. https://doi.org/10.1016/S0032-5910(99)00225-9

Hosseini, S., Patel, D., Ein-Mozaffari, F., \& Mehrvar, M. (2010). Study of solid-liquid mixing in agitated tanks through computational fluid dynamics modeling. Industrial and Engineering Chemistry Research, 49(9), 4426-4435. https://doi.org/10.1021/ie901130z

Hou, Y. Y., Wang, M., Holt, R., \& Williams, R. A. (2001). A Study Of The Mixing Characteristics of a Liquid Magnetically Stabilised Fluidised Bed Using Electrical Resistance Tomography. In 2nd World Congress on Industrial Process. Leeds: International Society for Industrial Process Tomography.

Hu, H. H. (1996). Direct simulation of flows of solid-liquid mixtures. International Journal of Multiphase Flow, 22(2), 335-352. https://doi.org/10.1016/0301-9322(95)00068-2

Hua, P., Woo, E. J., Webster, J. G., \& Tompkins, W. J. (1993). Using compound electrodes in electrical impedance tomography. IEEE Transactions on Bio-Medical Engineering, 40(1), 29-34. https://doi.org/10.1109/10.204768

Joseph, G.G., Zenit, R., Hunt, M.L., \& Rosenwinkel, A.M. (2001). Particle-wall collisions in a viscous fluid. Journal of Fluid Mechanics, 433, 329-346. https://doi.org/10.1017/S0022112001003470

Joshi, J. B., Deshpande, N. S., Dinkar, M., \& Phanikumar, D. V. (2001). Hydrodynamic stability of multiphase reactors. Advances in Chemical Engineering, 26, 1-130. https://doi.org/10.1016/S0065-2377(01)26002-5 
References

Kafui, K. D., Thornton, C., \& Adams, M. J. (2002). Discrete particle-continuum fluid modelling of gas-solid fluidised beds. Chemical Engineering Science, 57(13), 2395-2410. https://doi.org/10.1016/S0009-2509(02)00140-9

Kashyap, M., Chalermsinsuwan, B., \& Gidaspow, D. (2011). Measuring turbulence in a circulating fluidized bed using PIV techniques. Particuology, 9(6), 572-588. https://doi.org/10.1016/j.partic.2011.06.007

Kazemzadeh, A. (2016). Mixing of Complex Fluids with the Coaxial Mixers Composed of Two Central Impellers and an Anchor. Ryerson University. Toronto.

Kazemzadeh, A., Ein-Mozaffari, F., Lohi, A., \& Pakzad, L. (2016). Effect of the rheological properties on the mixing of Herschel-Bulkley fluids with coaxial mixers: Applications of tomography, CFD, and response surface methodology. Canadian Journal of Chemical Engineering, 94(12), 2394-2406. https://doi.org/10.1002/cjce.22601

Kazemzadeh, A., Ein-Mozaffari, F., Lohi, A., \& Pakzad, L. (2017a). Effect of Impeller Spacing on the Flow Field of Yield-Pseudoplastic Fluids Generated by a Coaxial Mixing System Composed of Two Central Impellers and an Anchor. Chemical Engineering Communications, 204(4), 453466. https://doi.org/10.1080/00986445.2016.1277520

Kazemzadeh, A., Ein-Mozaffari, F., Lohi, A., \& Pakzad, L. (2017b). Intensification of mixing of shear-thinning fluids possessing yield stress with the coaxial mixers composed of two different central impellers and an anchor. Chemical Engineering and Processing: Process Intensification, 111, 101-114. https://doi.org/10.1016/j.cep.2016.10.019

Kiared, K., Larachi, F., Cassanello, M., \& Chaouki, J. (1997). Flow Structure of the Solids in a Three-Dimensional Liquid Fluidized Bed. Industrial and Engineering Chemistry Research, 36(11), 4695-4704. https://doi.org/10.1021/ie970161k

Kirmizakis, P., Tsamoutsoglou, C., Kayan, B., \& Kalderis, D. (2014). Subcritical water treatment of landfill leachate: Application of response surface methodology. Journal of Environmental Management, 146, 9-15. https://doi.org/10.1016/j.jenvman.2014.04.037

Kunii, D., \& Levenspiel, O. (1977). Fluidization engineering. Krieger Pub. Co. Huntington, N.Y 
References

Launder, B. E., \& Spalding, D. B. (1974). The numerical computation of turbulent flows. Computer Methods in Applied Mechanics and Engineering, 3(2), 269-289. https://doi.org/10.1016/0045-7825(74)90029-2

Laverman, J. a., Roghair, I., van Sint Annaland, M., \& Kuipers, J. a. M. (2008). Investigation into the hydrodynamics of gas-solid fluidized beds using particle image velocimetry coupled with digital image analysis. The Canadian Journal of Chemical Engineering, 86(3), 523-535. https://doi.org/10.1002/cjce.20054

Li, J., \& Kuipers, J. A. M. (2002). Effect of pressure on gas-solid flow behavior in dense gasfluidized beds: a discrete particle simulation study. Powder Technology, 127(2), 173-184. https://doi.org/10.1016/S0032-5910(02)00116-X

Li, J., Langston, P. A., Webb, C., \& Dyakowski, T. (2004). Flow of sphero-disc particles in rectangular hoppers - A DEM and experimental comparison in 3D. Chemical Engineering Science, 59(24), 5917-5929. https://doi.org/10.1016/j.ces.2004.07.022

Li, X., Hunt, M. L., \& Colonius, T. (2012). A contact model for normal immersed collisions between a particle and a wall. Journal of Fluid Mechanics, 691, 123-145. https://doi.org/10.1017/jfm.2011.461

Limtrakul, S., Chen, J., Ramachandran, P. A., \& Duduković, M. P. (2005). Solids motion and holdup profiles in liquid fluidized beds. Chemical Engineering Science, 60(7), 1889-1900. https://doi.org/10.1016/j.ces.2004.11.026

Liu, G., Yu, F., Lu, H., Wang, S., Liao, P., \& Hao, Z. (2016). CFD-DEM simulation of liquidsolid fluidized bed with dynamic restitution coefficient. Powder Technology, 304, 186-197. https://doi.org/10.1016/j.powtec.2016.08.058

Lucas, A., Arnaldos, J., Casal, J., \& Pulgjaner, L. (1986). Improved Equation for the Calculation of Minimum Fluidization Velocity. Industrial and Engineering Chemistry Process Design and Development, 25(2), 426-429. https://doi.org/10.1021/i200033a013

Luding, S. (2008). Introduction to discrete element methods: Basic of contact force models and how to perform the micro-macro transition to continuum theory. European Journal of 
References

Environmental and Civil Engineering, $\quad$ 12(7-8), 785-826. https://doi.org/10.1080/19648189.2008.9693050

Lundberg, J., \& Halvorsen, B. M. (2008). A review of some exsisting drag models describing the interaction between phases in a bubbling fluidized bed. Proc. 49th Scand. Conf. Simulation and Modeling, Oslo University College, Oslo, Norway, 7-8.

Ma, J., Liu, D., \& Chen, X. (2015). Rotational behavior of dry spheres obliquely impacting on liquid layers. Powder Technology, $\quad$ 270(PB), $418-423$. https://doi.org/10.1016/j.powtec.2014.08.042

Malone, K. F., \& Xu, B. H. (2007). Numerical Investigation of the Layer-Inversion Phenomenon in Binary Solid Liquid Fluidized Beds. The $12^{\text {th }}$ International Conference of Fluidization - New Horizons in Fluidization Engineering. Vancouver, Canada.

Malone, K. F., \& Xu, B. H. (2008). Particle-scale simulation of heat transfer in liquid-fluidised beds. Powder Technology, 184(2), 189-204. https://doi.org/10.1016/j.powtec.2007.11.043

Malone, K. F., Xu, B. H., \& Fairweather, M. (2006). The combined-continuum-and-discretemodel (CCDM) for simulation of liquid-particle flows. Computer Aided Chemical Engineering, 21(C), 227-232. https://doi.org/10.1016/S1570-7946(06)80051-9

Mann, R., Dickin, F. J., Wang, M., Dyakowski, T., Williams, R. A., Edwards, R. B., \& Holden, P. J. (1997). Application of electrical resistance tomography to interrogate mixing processes at plant scale. Chemical Engineering Science, 52(13), 2087-2097. https://doi.org/10.1016/S00092509(97)00036-5

Mathiesen, V., Solberg, T., \& Hjertager, B. H. (2000). An experimental and computational study of multiphase flow behavior in a circulating fluidized bed. International Journal of Multiphase Flow, 26(3), 387-419. https://doi.org/10.1016/S0301-9322(99)00027-0

Mindlin, R. D., \& Deresiewicz, H. (1953). Elastic spheres in contact under varying oblique forces. American Society of Mechanical Engineers - Journal of Applied Mechanics. https://doi.org/10.1007/978-1-4613-8865-4_35 
Mishra, P., \& Ein-Mozaffari, F. (2016). Using tomograms to assess the local solid concentrations in a slurry reactor equipped with a Maxblend impeller. Powder Technology, 301, 701-712. https://doi.org/10.1016/j.powtec.2016.07.007

Mishra, P., \& Ein-Mozaffari, F. (2017). Using computational fluid dynamics to analyze the performance of the Maxblend impeller in solid-liquid mixing operations. International Journal of Multiphase Flow, 91, 194-207. https://doi.org/10.1016/j.ijmultiphaseflow.2017.01.009

Moreno-Casas, P. A., \& Bombardelli, F. A. (2016). Computation of the Basset force: recent advances and environmental flow applications. Environmental Fluid Mechanics, 16(1), 193-208. https://doi.org/10.1007/s10652-015-9424-1

Morrison, F. a. (2001). Understanding Rheology. Oxford University Press, 387-394. https://doi.org/10.3933/ApplRheol-12-233

Movafagh, H., Turcotte, G., \& Ein-Mozaffari, F. (2016). Using tomography images to study the mixing of wheat straw slurries. Biofuels, 7(4), 365-375. https://doi.org/10.1080/17597269.2015.1138038

Norouzi, H. R., Zarghami, R., Sotudeh-Gharebagh, R., \& Mostoufi, N. (2016). Coupled CFDDEM Modeling: Formulation, Implementation and Application to Multiphase Flows. Coupled CFD-DEM Modeling: Formulation, Implementation and Application to Multiphase Flows. https://doi.org/10.1002/9781119005315

Odar, F., \& Hamilton, W. S. (1964). Forces on a Sphere Accelerating in a Viscous Fluid. Journal of Fluid Mechanics, 18(2), 302-314. https://doi.org/10.4164/sptj1964.6.247

Pakzad, L., \& Azimi, A. H. (2017). Investigations on the dynamics of particle clouds in stagnant water using response surface methodology, Canadian Journal of Civil Engineering (44), 117-128.

Pakzad, L., Ein-Mozaffari, F., \& Chan, P. (2008). Using electrical resistance tomography and computational fluid dynamics modeling to study the formation of cavern in the mixing of pseudoplastic fluids possessing yield stress. Chemical Engineering Science, 63(9), 2508-2522. https://doi.org/10.1016/j.ces.2008.02.009 
References

Pakzad, L., Ein-Mozaffari, F., Upreti, S. R., \& Lohi, A. (2013). Characterisation of the mixing of non-newtonian fluids with a scaba 6SRGT impeller through ert and CFD. Canadian Journal of Chemical Engineering, 91(1), 90-100. https://doi.org/10.1002/cjce.21616

Pan, T.W., Joseph, D.D., Bai, R., Glowinski, R., \& Sarin, V. (2002). Fluidization of 1204 spheres: simulation and experiment. Journal of Fluid Mechanics, 451. https://doi.org/10.1017/S0022112001006474

Parker, D. J., Dijkstra, A. E., Martin, T. W., \& Seville, J. P. K. (1997). Positron emission particle tracking studies of spherical particle motion in rotating drums. Chemical Engineering Science, 52(13), 2011-2022. https://doi.org/10.1016/S0009-2509(97)00030-4

Peng, L., Ye, J., Lu, G., \& Yang, W. (2012). Evaluation of effect of number of electrodes in ECT sensors on image quality. IEEE Sensors Journal, 12(5), 1554-1565. https://doi.org/10.1109/JSEN.2011.2174438

Peng, Z., Ghatage, S. V., Doroodchi, E., Joshi, J. B., Evans, G. M., \& Moghtaderi, B. (2014). Forces acting on a single introduced particle in a solid-liquid fluidised bed. Chemical Engineering Science, 116, 49-70. https://doi.org/10.1016/j.ces.2014.04.040

Peng, Z., Joshi, J. B., Moghtaderi, B., Khan, M. S., Evans, G. M., \& Doroodchi, E. (2016). Segregation and dispersion of binary solids in liquid fluidised beds: A CFD-DEM study. Chemical Engineering Science, 152, 65-83. https://doi.org/10.1016/j.ces.2016.05.032

Porzuczek, J. (2014). Applications of Electrical Capacitance Tomography for research on phenomena occurring in the fluidised bed reactors. Chemical and Process Engineering - Inzynieria Chemiczna I Procesowa, 35(4), 397-408. https://doi.org/10.2478/cpe-2014-0030

Potter, O., \& Nicklin, D. J. (1992). Fluidization VII. In Proceedings of the Seventh Engineering Foundatian Conference on Fluidization May 3-8 (pp. 259-260). Brisbane, Australia.

Razzak, S. A., Barghi, S., \& Zhu, J. X. (2007). Electrical resistance tomography for flow characterization of a gas-liquid-solid three-phase circulating fluidized bed. Chemical Engineering Science, 62(24), 7253-7263. https://doi.org/10.1016/j.ces.2007.08.057 
Razzak, S. A., Barghi, S., \& Zhu, J. X. (2010). Axial hydrodynamic studies in a gas-liquid-solid circulating fluidized bed riser. Powder Technology, 199(1), 77-86. https://doi.org/10.1016/j.powtec.2009.05.014

Razzak, S. A., Barghi, S., Zhu, J. X., \& Mi, Y. (2009). Phase holdup measurement in a gas-liquidsolid circulating fluidized bed (GLSCFB) riser using electrical resistance tomography and optical fibre probe. Chemical Engineering Journal, 147(2-3), 210-218. https://doi.org/10.1016/j.cej.2008.07.022

Robinson, M., Ramaioli, M., \& Luding, S. (2014). Fluid-particle flow simulations using two-waycoupled mesoscale SPH-DEM and validation. International Journal of Multiphase Flow, 59, 121134. https://doi.org/10.1016/j.ijmultiphaseflow.2013.11.003

Rubinow, S. I., \& Keller, J. B. (1961). The transverse force on a spinning sphere moving in a viscous fluid. Journal of Fluid Mechanics, 11(3), 447-459. https://doi.org/10.1017/S0022112061000640

Saffman, P. G. (1965). The lift on a small sphere in a slow shear flow. Journal of Fluid Mechanics, 22(2), 385-400. https://doi.org/10.1017/S0022112065000824

Schiller, L., \& Naumann, Z. (1933). A drag coefficient correlation. Z.Ver.Deutsch.Ing, 77(13-14), 318-320. https://doi.org/10.1016/j.ijheatmasstransfer.2009.02.006

Seibert, K. D., \& Burns, M. A. (1998). Simulation of structural phenomena in mixed-particle fluidized beds. AIChE Journal, 44(3), 528-537. https://doi.org/10.1002/aic.690440304

Sen, M., Barrasso, D., Singh, R., \& Ramachandran, R. (2014). A Multi-Scale Hybrid CFD-DEMPBM Description of a Fluid-Bed Granulation Process. Processes, 2(1), 89-111. https://doi.org/10.3390/pr2010089

Sharifi, M., \& Young, B. (2013). Electrical resistance tomography (ert) applications to chemical engineering. Chemical Engineering Research and Design, 91(9), 1625-1645. https://doi.org/10.1016/j.cherd.2013.05.026

Shuai, W., Guodong, L., Huilin, L., Pengfei, X., Yunchao, Y., \& Gidaspow, D. (2012). A cluster structure-dependent drag coefficient model applied to risers. Powder Technology, 225, 176-189. https://doi.org/10.1016/j.powtec.2012.04.006 
Sun, J., \& Yan, Y. (2016). Non-intrusive measurement and hydrodynamics characterization of gassolid fluidized beds: A review. Measurement Science and Technology, 27(11), 112001. https://doi.org/10.1088/0957-0233/27/11/112001

Sutkar, V. S., Deen, N. G., Salikov, V., Antonyuk, S., Heinrich, S., \& Kuipers, J. A. M. (2015). Experimental and numerical investigations of a pseudo-2D spout fluidized bed with draft plates. Powder Technology, 270(PB), 537-547. https://doi.org/10.1016/j.powtec.2013.11.030

Syamlal, M., \& O’Brien, T. J. (1989). Computer simulation of bubbles in a fluidized bed. AIChE Symposium Series, 85(270), 22-31. https://doi.org/10.2307/302397

Tu, J., Yeoh, G.-H., \& Liu, C. (2013). Computational Fluid Dynamics A Practical Approach (2nd ed.). Waltham, MA: Elsevier.

Van der Hoef, M. A., Van Sint Annaland, M., Deen, N. G., \& Kuipers, J. A. M. (2008). Numerical Simulation of Dense Gas-Solid Fluidized Beds: A Multiscale Modeling Strategy. Annual Review of Fluid Mechanics, 40(1), 47-70. https://doi.org/10.1146/annurev.fluid.40.111406.102130

Van der Hoef, M. A., Van Sint Annaland, M., \& Kuipers, J. a M. (2004). Computational fluid dynamics for dense gas-solid fluidized beds: a multi-scale modeling strategy. Chem. Eng. Sci., 59(22-23), 5157-5165. https://doi.org/10.1016/j.ces.2004.07.013

Van der Hoef, M. A., Ye, M., Van Sint Annaland, M., Andrews, A. T., Sundaresan, S., \& Kuipers, J. A. M. (2006). Multiscale Modeling of Gas-Fluidized Beds. Advances in Chemical Engineering. https://doi.org/10.1016/S0065-2377(06)31002-2

Versteeg, H. K., \& Malalasekera, W. (1995). An Introduction to Computational Fluid Dynamics The Finite Volume Method. Fluid Flow Handbook. New York: John Wiley \& Sons. https://doi.org/10.2514/1.22547

Viergever, M. (1988). Mathematics and Computer Science in Medical Imaging. Berlin: Springer International Publishing.

Wallis, G. (1962). one-dimensional waves in two-component flow (with particular reference to the stability of fluidized beds). UKAEA Report AEEW-R162. 
Wang, S., Guo, S., Gao, J., Lan, X., Dong, Q., \& Li, X. (2012). Simulation of flow behavior of liquid and particles in a liquid-solid fluidized bed. Powder Technology, 224, 365-373. https://doi.org/10.1016/j.powtec.2012.03.022

Wang, S., Sun, Z., Li, X., Gao, J., Lan, X., \& Dong, Q. (2013). Simulation of flow behavior of particles in liquid-solid fluidized bed with uniform magnetic field. Powder Technology, 237, 314325. https://doi.org/10.1016/j.powtec.2012.12.013

Werther, J. (1999). Measurement Techniques in Fluidized Beds. Powder Technology, 102, 15-36. https://doi.org/10.1016/S0032-5910(98)00202-2

Xu, B. H., \& Yu, a. B. (1997). Numerical simulation of the gas-solid flow in a fluidized bed by combining discrete particle method with computational fluid dynamics. Chemical Engineering Science, 52(16), 2785-2809. https://doi.org/10.1016/S0009-2509(97)00081-X

Yu, Z., Zhu, B., Cao, S., \& Liu, Y. (2014). Effect of virtual mass force on the mixed transport process in a multiphase rotodynamic pump. Advances in Mechanical Engineering, 2014. https://doi.org/10.1155/2014/958352

Zhao, Y., Ding, Y., Wu, C., \& Cheng, Y. (2010). Numerical simulation of hydrodynamics in downers using a CFD-DEM coupled approach. Powder Technology, 199(1), 2-12. https://doi.org/10.1016/j.powtec.2009.04.014

Zhou, H., Flamant, G., Gauthier, D., \& Lu, J. (2004). Numerical simulation of the turbulent gasparticle flow in a fluidized bed by an LES-DPM model. Chemical Engineering Research and Design, 82(7), 918-926. https://doi.org/10.1205/0263876041596788

Zhou Y.C., Wright, B.D., Yang, Y.Y., Xu, B.H., Yu, A.B. (1999). Y.C. Zhou. Rolling friction in the dynamic simulation of sandpile formation. Physica A: Statistical Mechanics and Its Applications, 269, 536-553. https://doi.org/0378-4371/99

Zhu, H. P., Zhou, Z. Y., Yang, R. Y., \& Yu, A. B. (2007). Discrete particle simulation of particulate systems: Theoretical developments. Chemical Engineering Science, 62(13), 3378-3396. https://doi.org/10.1016/j.ces.2006.12.089 